Review

\title{
Camelid single-domain antibody fragments: Uses and prospects to investigate protein misfolding and aggregation, and to treat diseases associated with these phenomena
}

\author{
Coralie Pain, Janice Dumont, Mireille Dumoulin* \\ Laboratory of Enzymology and Protein Folding, Centre for Protein Engineering, University of Liège, Liège, Belgium
}

\section{A R T I C L E I N F O}

\section{Article history:}

Received 8 September 2014

Accepted 23 January 2015

Available online 3 February 2015

\section{Keywords:}

Variable domain of heavy-chain antibody

Inhibition of protein misfolding and aggregation

Protein misfolding diseases

Amyloidoses

$\mathrm{V}_{\mathrm{H}} \mathrm{H}$

Nanobody

\begin{abstract}
A B S T R A C T
The deposition of misfolded peptides and proteins in the form of amyloid fibrils is the hallmark of nearly fifty medical disorders, including Alzheimer's disease, Parkinson's disease, prion diseases and type II diabetes. These disorders, referred to as amyloidoses, generally become apparent late in life. Their psycho-sociological and economic incidence in western societies will be therefore considerable in the coming decades due to the ageing of the population. Neither preventing nor curative treatments are available yet. These disorders constitute therefore a medical challenge of great importance. Thus, an extensive research is being carried out to understand, at the molecular level, (i) how amyloidogenic proteins misfold and convert from their soluble form into amyloid fibrils, and (ii) how these aggregates or some of their oligomeric precursor species are toxic. The formation of amyloid fibrils proceeds through a complex nucleation/polymerisation mechanism with the formation of various species, including small oligomers. In this review, we focus on how $\mathrm{V}_{\mathrm{H}} \mathrm{Hs}$ or nanobodies, the antigen-binding domains of camelid heavy-chain antibodies, are being increasingly used to characterise each of the species formed on the pathway of fibril formation in terms of structure, stability, kinetics of formation and toxicity. We first introduce the characteristic features of nanobodies compared to those of conventional antibody fragments. Thereafter, we discuss how nanobodies, due to their unique properties, are used as probes to dissect the molecular mechanisms of misfolding and aggregation of six proteins associated with diseases, i.e. human lysozyme, $\beta 2$-microglobulin, $\alpha$-synuclein, prion, polyadenylate binding protein nuclear 1 and amyloid $\beta$-peptide. A brief general presentation of each disease and the associated peptide/protein is also provided. In addition, we discuss how nanobodies could be used as early diagnostic tools and as novel strategies to treat diseases associated with protein misfolding and aggregation.
\end{abstract}

๑ 2015 Elsevier B.V. and Société Française de Biochimie et Biologie Moléculaire (SFBBM). All rights reserved.

\footnotetext{
Abbreviations: Ab, antibody; $\mathrm{A} \beta$, amyloid $\beta$-peptide; AD, Alzheimer's disease; ANS, anilino naphthalene-sulfonic acid; AP, alkaline phosphatase; APP, amyloid precursor protein; $\alpha$ Syn, $\alpha$-synuclein; $\beta 2 \mathrm{~m}, \beta 2$-microglobulin; BBB, blood-brain barrier; $C D R$, complementarity determining region; $C_{m}$, concentration of mid-denaturation; $C R$, Congo red; CSF, cerebrospinal fluid; $\triangle \mathrm{N} 6 \beta 2 \mathrm{~m}$, a truncated form of $\beta 2 \mathrm{~m}$ lacking the six N-terminal amino acids; DLS, dynamic light scattering; DRA, dialysis-related amyloidosis; FR, framework; FTIR, Fourier transform infrared spectroscopy; Fv, variable fragment made of the VH and VL domains of conventional antibodies; GFP, green fluorescent protein; HCAb, heavy-chain antibody; H/D, hydrogen/deuterium; HSQC, heteronuclear single quantum coherence; HuL, human lysozyme; IAPP, islet amyloid polypeptide; IMAC, immobilised metal ion affinity chromatography; ITC, isothermal titration calorimetry; LB, Lewy bodies; MCH1, light chain of the type I major histocompatibility complex; MM, molecular mass; NAC, non-A $\beta$ component; NLS, nuclear localisation signal; NMR, nuclear magnetic resonance; OPMD, oculopharyngeal muscular dystrophy; PABPN1, polyadenylate binding protein nuclear 1; PD, Parkinson's disease; PrP, prion protein; $\operatorname{PrP}^{\mathrm{C}}$, normal cellular prion protein; $\operatorname{Pr} \mathrm{P}^{\mathrm{Sc}}$, misfolded form of the prion protein associated with disease; QCM, quartz crystal microbalance; sdAb, single-domain antibody; SPR, surface plasmon resonance; scFv, Single-chain variable fragment made of the VH and VL domains of conventional antibodies tethered via an oligopeptide; ThT, thioflavin-T; TEM, transmission electron microscopy; $\mathrm{T}_{\mathrm{m}}$, temperature of mid-denaturation; TSEs, transmissible spongiform encephalopathies; $\mathrm{V}_{\mathrm{H}}$, variable domain of the heavy chain of conventional antibodies; $\mathrm{V}_{\mathrm{H}} \mathrm{H}$, variable domain of heavy-chain antibodies; $\mathrm{V}_{\mathrm{L}}$, variable domain of the light chain of conventional antibodies.

* Corresponding author. Laboratory of Enzymology and Protein Folding, Centre for Protein Engineering, Allée de la Chimie 3, University of Liège, 4000 Liège, Belgium. Tel.: +32 (0)4 3663546 .

E-mail address: mdumoulin@ulg.ac.be (M. Dumoulin).
} 


\section{Introduction}

The amino acid sequence of each protein newly synthetised by ribosomes contains all the information required for the protein to acquire its native tridimensional structure and, therefore, its functional biological state. Nature has in fact developed sophisticated mechanisms to ensure the correct folding of proteins, including the evolution of specific polypeptidic sequences or the involvement of factors such as folding catalysts and molecular chaperones [1]. The latter help the polypeptide chains to correctly and efficiently fold but they do not however determine the final native conformation of the protein. Despite these mechanisms, some proteins, however, fail to fold correctly or to maintain their native state, because of different factors such as mutations or increased concentration. These proteins are normally rapidly recognised by the quality control system and degraded by the proteasome or other proteolytic machineries [2]. In spite of these quality control and degradation machineries, the probability that some proteins fail to adopt or to maintain their functional conformation is however not negligible. Given the crucial roles of proteins in virtually any cellular processes, this failure generally leads to serious diseases which are known as protein conformational diseases or protein misfolding diseases $[3,4]$. In some cases, not only misfolded proteins are not anymore able to exert their normal biological activity (and this could be the direct cause of some diseases such as in cystic fibrosis [1]), but they can also interact with each other to form pathological insoluble, highly organised and stable aggregates called amyloid fibrils. The formation of such aggregates is associated with nearly fifty diseases referred to as amyloidoses [3].

Amyloidoses are neurodegenerative or non-neuropathic, depending on whether the amyloid fibrils deposit in the nervous system or in other tissues or organs; in the latter case, the disease can be localised (i.e. affecting only one organ) or systemic (i.e. affecting various organs) [3]. The most common neurodegenerative amyloidoses are Alzheimer's disease (AD) and Parkinson's disease (PD). Amyloidoses generally appear late in life; due to the rising ageing of the population, they inflict enormous psycho-sociological and economic burdens on western societies and constitute therefore a great medical challenge. These age-associated disorders indeed affect several million people per year. For example, it is estimated that, in 2005, between 4.1 and 4.6 million individuals were living with Parkinson's disease in the 15 more populated nations worldwide, and this number will double by 2030 [5]. According to a report published in 2013, Alzheimer's disease is the sixth leading cause of all deaths in the United States. An estimated 5.2 million Americans have AD and, by 2050, the incidence of AD is expected to approach nearly a million new cases per year, with a total estimated prevalence of 13.8 million people [6]. So far, there is no preventive or curative therapy for these devastating pathologies, and the current treatments only alleviate the symptoms without modifying the course of the diseases. For example, in the case of AD, acetylcholinesterase inhibitors are used to reduce the loss of acetylcholine caused by the death of cholinergic neurons [7]. For the patients suffering from PD, most treatments aim at correcting the deficiency of the brain neurotransmitter dopamine [8]. There is therefore an intensive worldwide research effort to shed light on the mechanisms, at the molecular level, of protein misfolding and aggregation and their link to pathology. The identification of all the various species formed during the complex process of fibril formation as well as their characterisation in terms of structure, stability, kinetics of formation and toxicity are essential to develop some early diagnostic tools and to identify targets for therapeutic treatments.

Each amyloidosis is associated with the aggregation of a particular peptide or protein into amyloid fibrils (for example
$A \beta$-peptide $(A \beta)$ and Tau protein in Alzheimer's disease, $\alpha$-synuclein ( $\alpha$ Syn) in Parkinson's diseases and islet amyloid polypeptide (IAPP) in type II diabetes). The disease-associated proteins differ widely in their sequence, size and native structure; in their aggregated form, however they all share a similar highly organised structure, rich in $\beta$-sheets and known as cross$\beta$ structure [9]. Fibril formation is a complex phenomenon, generally described as a nucleation/polymerisation process, characterised by a sigmoidal growth profile (Fig. 1). This process involves a variety of conformational rearrangements and multiple steps of assembly [3,4]. First, a slow nucleation step is characterised by a lag phase; it involves the misfolding of the proteins into aggregation prone species (also referred to as amyloidogenic intermediate) and the association of these species into various oligomeric species, leading to the formation of nuclei or seeds of aggregation. These oligomers, also called prefibrillar species, are structurally diverse and some of them have been found to be more cytotoxic than mature fibrils [10]. During the polymerisation step (or elongation phase), the nuclei rapidly expand by addition of monomers or oligomers to form protofibrils and finally fibrils. In the final steady state or stationary phase, fibrils and monomers are in equilibrium and the structure of the fibrils can still evolve for example by rearrangement of the region of the protein that is not part of the core of the fibrils, leading to mature fibrils [11]. Besides, the amyloid fibrils can also associate laterally [12]. The lag phase can be shortened or even suppressed by the addition of seeds (i.e. preformed oligomers or fibrils), a phenomenon known as seeding [13]. Moreover, increasing evidence suggests that once a critical concentration of fibrils has formed, the primary nucleation/polymerisation pathway described above can be dominated by secondary pathway(s) such as fibril fragmentation, lateral growth and/or fibril-catalysed secondary nucleation [14-16]. Finally, recent findings suggest a prion-like spreading of amyloidogenic proteins: aggregates of tau, $\alpha$ Syn, $A \beta$ and huntingtin have indeed been found to be capable of moving between cells and trigger the misfolding of their normal conformers [17].

Because of their insoluble character, their heterogeneity in size and quantity, and/or their transient feature, the characterisation of most of the species formed upon fibril formation is extremely challenging. Indeed, these species cannot be easily studied by most of the conventional techniques available for soluble proteins because these require concentrated and homogeneous samples which are difficult to obtain for each individual intermediate species. Since a given protein generally adopts a different conformation in its native, oligomeric and amyloid forms, the development of specific and sensitive structural (or conformational) molecular probes for the characterisation of these distinct forms is therefore a very valuable approach. Monoclonal conformational antibodies can be extremely promising for this purpose since they could in principle be specifically raised against any conformational state of peptides or proteins [18-21]. Moreover, as illustrated in this review, the extent of their specificity can be adjusted through the judicious choice of antigens and of the screening procedures.

Antibodies can be used in several ways to investigate the mechanism of fibril formation. For example, the binding properties of a series of antibodies specifically raised against various epitopes at the surface of the native state of a protein can be compared before and after the conformational changes leading successively to amyloidogenic intermediates, oligomeric species and mature fibrils $[21,22]$. In this way, the regions of the protein that are structurally reorganised can be identified at each step. Alternatively, partial unfolding events expose to the solvent some regions of the protein, normally buried within the native fold; antibodies raised against such epitopes will therefore recognise amyloidogenic 


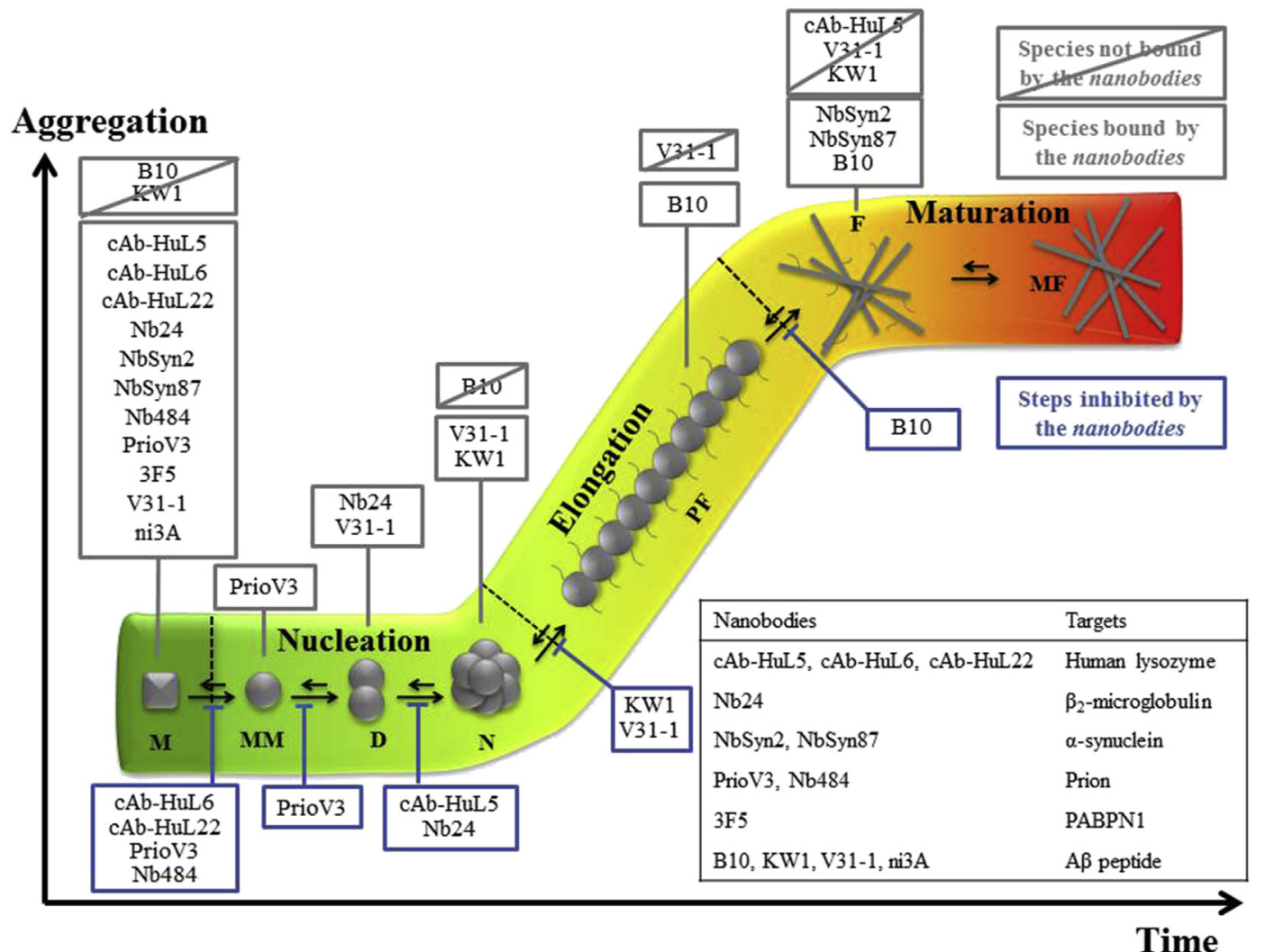

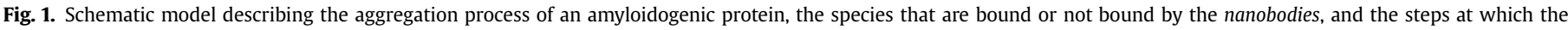

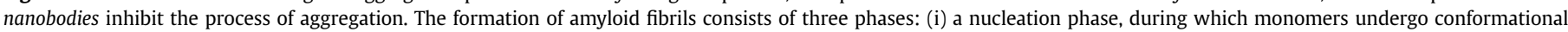

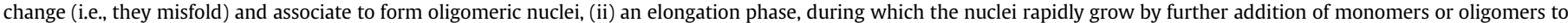

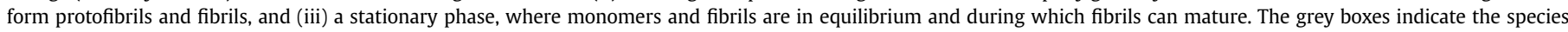

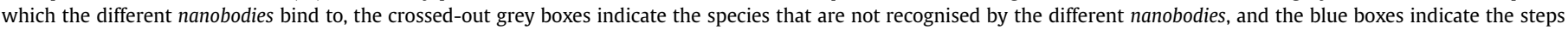
inhibited by the various nanobodies. M, monomers; MM, misfolded monomers; D, dimers; N, nuclei; PF, protofibrils; F, fibrils; MF, mature fibrils.

intermediates but not the native conformation of the protein. Thus, such an approach can provide information on the structure which is adopted by the region(s) of the protein involved in the unfolding event [21]. Finally, antibodies recognising epitopes that are generic of oligomeric species or amyloid fibrils have also been described [23-26]. Thus, from a mechanistic point of view, the use of these various specific antibodies could in principle allow to map the apparition of each species in time and can allow a detailed comparison of the aggregation pathways under different conditions including events occurring in vivo [27].

Several antibody formats have been used as structural and mechanistic probes to investigate the aggregation process of diverse proteins: whole mice monoclonal antibodies [28], antibody fragments such as Fab [25], scFv [29,30] and $\mathrm{V}_{\mathrm{H}}[31]$, and recently, the variable domains of heavy-chain antibodies, referred to as nanobodies or $\mathrm{V}_{\mathrm{H}} \mathrm{Hs}$. In this review, we report the main results of the studies involving the latter antibody format. We first introduce the characteristic features of nanobodies compared to those of conventional antibody fragments such as Fab, Fv and scFv. Then, we discuss how nanobodies, thanks to their unique properties, have been used as structural and mechanistic probes to dissect the mechanisms of misfolding and aggregation of a series of proteins associated with amyloid or conformational diseases. This last part is divided into six sections corresponding to six targets, i.e. human lysozyme (HuL), $\beta 2$-microglobulin $(\beta 2 \mathrm{~m}), \alpha-$ synuclein $(\alpha \operatorname{Syn})$, prion (PrP), polyadenylate binding protein nuclear 1 (PABPN1) and amyloid $\beta$-peptide $(A \beta)$. In addition, we discuss how nanobodies can be used as early diagnostic tools and to design novel strategies to treat diseases associated with amyloid fibril formation.

\section{Unique properties of nanobodies}

\subsection{Nanobody, the smallest natural antigen-binding fragment}

Nanobodies correspond to the variable domains of camelid heavy-chain antibodies (HCAbs). HCAbs were discovered in 1989 by the group of R. Hamers [32] during a practical experiment conducted by undergraduate students at the Free University of Brussels, Belgium [33]. These IgG antibodies lack light chains and the first constant domain of the heavy chains (i.e., the $\mathrm{CH} 1$ domains) present in their conventional counterparts; HCAbs are composed therefore of only two heavy chains (Fig. 2, central part). They are synthetised by Camelidae (including camels, llamas and dromedaries) in addition to conventional antibodies and their proportion depends on the species: HCAbs represent $50 \%$ of the IgG in dromedaries and camels and 25-45\% in llamas [32,34,35]. The antigenbinding domain of HCAbs is naturally reduced to a single domain of $\sim 130$ amino acids ( $14 \mathrm{kDa})$ and it therefore corresponds to the smallest available natural antigen-binding fragment; it is referred to as nanobody, $\mathrm{V}_{\mathrm{H}} \mathrm{H}$ or single-domain antibody ( $\mathrm{sdAb}$ ). In spite of their small size, nanobodies bind to their antigens with high affinity (i.e. in the nanomolar range) and specificity [36,37]. In this review, we use the term $\mathrm{V}_{\mathrm{H}} \mathrm{Hs}$ when structural features are concerned, otherwise we use the denomination nanobodies. 


\subsection{Structure and adaptations of $V_{H} H s$}

The tridimensional structure of $\mathrm{V}_{\mathrm{H}} \mathrm{Hs}$ corresponds to a typical immunoglobulin fold. It is composed of nine $\beta$-strands organised into two $\beta$-sheets, stabilised by a conserved disulphide bridge between Cys23 and Cys94 (IMGT numbering [38]) (Fig. 2, central part). The amino acid sequence of $\mathrm{V}_{\mathrm{H}} \mathrm{Hs}$ presents a high degree of identity $(\sim 80 \%)$ with the human $\mathrm{V}_{\mathrm{H}} 3$ (variable domain of the heavy chain of conventional antibodies) gene family [34,39,40]; it has however evolved to compensate for the absence of the $V_{L}$ domain as follows:

(i) Four hydrophobic amino acids, conserved among all $\mathrm{V}_{\mathrm{H}} \mathrm{s}$, are substituted by more hydrophilic residues in the $\mathrm{V}_{\mathrm{H}} \mathrm{H}$ sequences (V42F or V42Y, G49E, L50R and W52G, IMGT numbering) [41,42] (Fig. 2, central part). These amino acids are located in the framework 2 (FR2), corresponding to the region of the $V_{H}$ domain that interacts with the $V_{L}$ (variable domain of the light chain) domain in conventional antibodies. By decreasing the hydrophobicity of the surface of
$\mathrm{V}_{\mathrm{H}} \mathrm{H}$ that is exposed to the solvent due to the absence of the $\mathrm{V}_{\mathrm{L}}$, these mutations allow $\mathrm{V}_{\mathrm{H}} \mathrm{Hs}$ to be soluble in the absence of the $\mathrm{V}_{\mathrm{L}}$ domain.

(ii) The complementarity determining regions (CDRs, i.e. regions of the antibody that interact with the antigen) of $\mathrm{V}_{\mathrm{H}} \mathrm{Hs}$ are longer than those of $\mathrm{V}_{\mathrm{H}} \mathrm{s}$, and particularly the CDR1 and CDR3 (Fig. 2, central part). The average length of the CDR3 from human $\mathrm{V}_{\mathrm{H}} \mathrm{S}$ is 12 residues while that from dromedary $\mathrm{V}_{\mathrm{H}} \mathrm{Hs}$ contains most frequently $\sim 16-18$ amino acids $[34,43]$. Several $\mathrm{V}_{\mathrm{H}} \mathrm{Hs}$ with CDR3 longer than 25 residues have been reported [44,45]. The larger size of the CDRs is believed to compensate for the absence of the three CDRs from the $V_{L} S$ (the antigen is recognised by only three CDRs instead of six in conventional antibodies) and therefore provide a sufficiently large antigeninteracting surfaces of $600-800 \AA^{2}$ [46]. CDRs of $\mathrm{V}_{\mathrm{H}} \mathrm{Hs}$ also adopt a larger variety of conformations, compared to the CDRs of $V_{H^{S}}$ [47-49]. Together, these two characteristics allow a broad antigen-binding repertoire, counterbalancing the lack of $\mathrm{V}_{\mathrm{H}}-\mathrm{V}_{\mathrm{L}}$ combinatorial diversity.

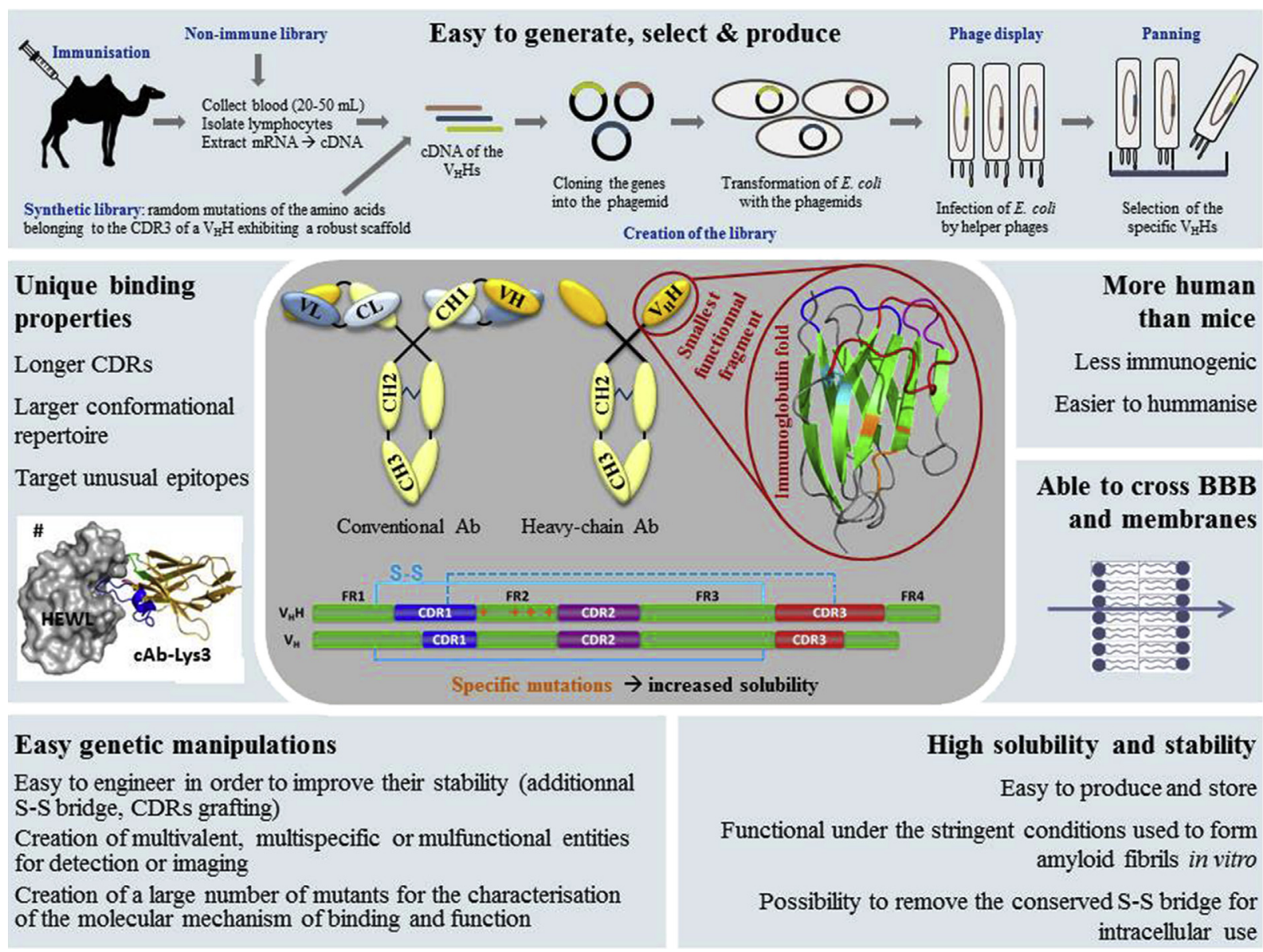

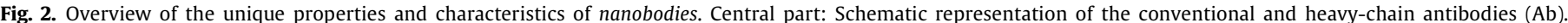

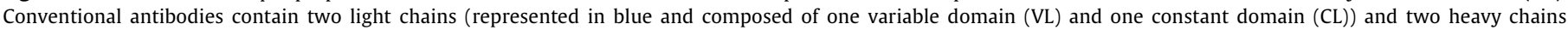

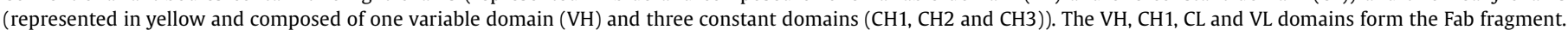

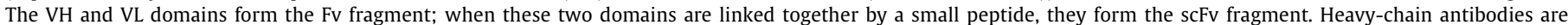

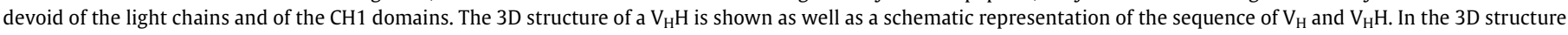

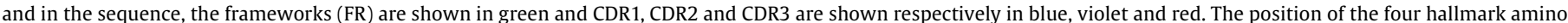

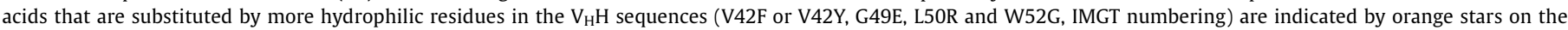

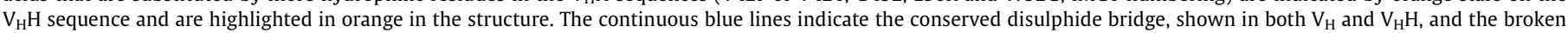

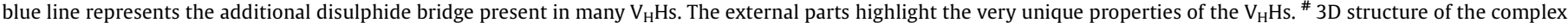

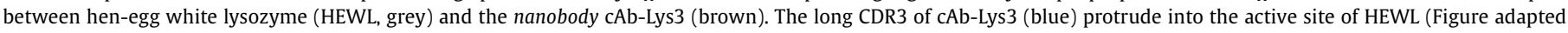
from Ref. [50]). 
Moreover, the long CDR3 can form a protruding loop, allowing $\mathrm{V}_{\mathrm{H}} \mathrm{Hs}$ to bind unique conformational epitopes, such as cryptic epitopes, clefts or cavities that are generally inaccessible to conventional $\mathrm{VH}-\mathrm{VL}$ pairs [50] (Fig. 2, left part, \#). Since this kind of epitopes is often associated with the function of proteins (e.g. enzyme active sites), nanobodies can thus be used to modulate biological activities [51]. As the CDR3 generally contributes from 50 to $100 \%$ of the antigen-interacting surface [34], it is easy to design and synthetise constrained peptides that mimic the CDR3 structure and retain the specificity/activity of the nanobody (i.e. enzyme inhibitors, receptor agonists or antagonists) [52].

(iii) An additional disulphide bond is present in many $\mathrm{V}_{\mathrm{H}} \mathrm{H}$ sequences, especially those originating from dromedaries; it is generally located between CDR1 and CDR3 (64\%) but can also be located between CDR3 and FR2 [39]. In general, $\mathrm{V}_{\mathrm{H}} \mathrm{Hs}$ with longer CDR3 have a higher probability to contain an additional disulphide bridge [37]. This disulphide bond probably restricts the flexibility of long CDRs, which is expected to be entropically counterproductive for binding, and therefore allows a strong interaction [53]. Moreover, it may also enable CDRs to adopt new conformations enabling $\mathrm{V}_{\mathrm{H}} \mathrm{Hs}$ to recognise an increased variety of epitopes [40,54].

\subsection{Nanobodies are easy to generate, select and produce}

Nanobodies can be obtained from immune, naive or synthetic libraries [34] (Fig. 2, upper part). In the first case, an animal is immunised with the antigen of interest and, after 5 or 6 weekly injections, lymphocytes are isolated from the peripheral blood. cDNAs are prepared from total extracted RNA, and those encoding $\mathrm{V}_{\mathrm{H}} \mathrm{H}$ genes are selected by PCR as described by Muyldermans [34]. Since the entire antigen-binding fragment of HCAb consists of one domain, encoded by a gene fragment of only $\sim 360$ bp that is easily amplified by PCR, small libraries of $\sim 10^{6}-10^{7}$ individual transformants are already representative of the immune repertoire [46]. Naive libraries are generated in the same manner from the blood of a non-immunised animal, while synthetic libraries are created, for example, by randomly mutating the amino acids belonging to CDR3 of a $\mathrm{V}_{\mathrm{H}} \mathrm{H}$ exhibiting a robust scaffold [55,56]. Some residues of the $\mathrm{CDR} 3$, that are essential to maintain its conformation, must be conserved to ensure the proper folding of the mutant $\mathrm{V}_{\mathrm{H}} \mathrm{Hs}$ [34]. The use of naive or synthetic libraries is particularly interesting in the case of toxic, pathogenic or nonimmunogenic antigens, or for antigens that are not available in sufficient quantities for immunisation [34]. They need however to be much larger $\left(\sim 10^{9}\right.$ individual clones) than immune libraries to allow the retrieval of high-affinity binders [46]; once prepared, the advantage of these libraries is that they can in theory be used to select binders against any target.

In all cases, antigen-specific nanobodies are retrieved from these libraries by phage display or other selection protocols such as bacterial display [57], yeast display [58] or ribosome display [59]. Due to its robustness, the phage display method is the most often used one. The $\mathrm{V}_{\mathrm{H}} \mathrm{H}$ genes, amplified by PCR, are generally cloned into an appropriate vector (phagemid) at the $3^{\prime}$-end of a gene coding for a coat protein of a filamentous phage (generally M13) [34]. $\mathrm{V}_{\mathrm{H}} \mathrm{Hs}$ are then expressed at the tip surface of the phages and the most specific binders are panned on antigens which are most of the time immobilised in wells of a microtiter plate by passive adsorption or biotinylated on streptavidincoated solid supports. Generally, two to three rounds of panning are sufficient to enrich the population of antigen- specific nanobodies compared to the non-specific ones. Nanobodies repertoires present a great advantage over those of conventional antibodies: they do not need the random combination of $\mathrm{V}_{\mathrm{H}}$ and $\mathrm{V}_{\mathrm{L}}$ chains which lead to the generation of many nonproductive combinations due to the loss of the original pairing [34]. Moreover, the whole procedure is also much less time and money consuming.

Finally, the selected nanobodies can be expressed recombinantly with a high yield in bacteria ( 40-70 mg per litre of culture) $[60,61]$, yeast ( $>100 \mathrm{mg}$ per litre of culture) [62,63] or plants (up to $30 \%$ total leaf proteins) $[64,65]$. Their purification is usually achieved by immobilised metal ion affinity chromatography (IMAC) via an engineered C-terminal $\mathrm{His}_{6}$ tag, or by other affinity chromatographies as nanobody gene is easily cloned in another vector to change the tag [60].

\subsection{Nanobodies are highly stable entities}

Nanobodies exhibit a high resistance to temperature (temperature of mid-denaturation, $\mathrm{T}_{\mathrm{m}}$, generally comprised between 60 and $80{ }^{\circ} \mathrm{C}$ ), chaotropic reagents (concentration of middenaturation, $C_{m}$, generally comprised between 2.3 and $3.3 \mathrm{M}$ guanidinium chloride and between 5.8 and $9 \mathrm{M}$ urea) or high pressure (pressure of mid-denaturation comprised between 600 and $750 \mathrm{MPa})$, and have high conformational stabilities $\left(\Delta \mathrm{G}^{\circ}\right.$ $\left(\mathrm{H}_{2} \mathrm{O}\right)$ comprised between 30 and $\left.60 \mathrm{~kJ} \mathrm{~mol}^{-1}\right)$ [37,66]. Consequently, nanobodies can be stored at $4{ }^{\circ} \mathrm{C}$ for months and at $-20{ }^{\circ} \mathrm{C}$ for years without losing their ability to bind to their antigen. They can also tolerate incubation at $37{ }^{\circ} \mathrm{C}$ for a long period (i.e. more than one week) in PBS [67] or even in plasma (i.e. no decrease in activity is observed after an incubation for $44 \mathrm{~h}$ in mouse plasma, [68]). The fact that nanobodies exhibit a high stability is especially important for their use as molecular probes to study the process of amyloid fibril formation because denaturing conditions and/or long incubation time (up to several weeks) need generally to be used to trigger the in vitro aggregation of target proteins. Finally, their high stability allows most of nanobodies to be easily concentrated to $1-15 \mathrm{mg} \mathrm{mL}^{-1}$ in standard buffers [46] and to be freeze-dried without losing their functionality $[37,69]$.

\subsection{The properties of nanobodies are easily improved using protein engineering}

Since $\mathrm{V}_{\mathrm{H}} \mathrm{Hs}$ are composed of only one single-domain of $\sim 130$ amino acids, and thus are encoded by a short gene fragment, their properties (i.e. affinity, stability, immunogenicity, etc.) can be easily and straightforwardly improved by protein engineering in comparison with the more complex conventional antibody fragments such as Fab and scFv. For example, the stability of a particular

Table 1

Affinity of HuL-specific nanobodies and their engineered counterparts for Wt-HuL. The affinity and the $\mathrm{T}_{\mathrm{m}}$ of the nanobodies under buffer conditions $(\mathrm{pH} 5.5,3 \mathrm{M}$ urea and $48^{\circ} \mathrm{C}$ ) used to induce the formation of amyloid fibrils by the I56T and D67H variants of human lysozyme is also shown.

\begin{tabular}{lclc}
\hline $\mathrm{V}_{\mathrm{H}} \mathrm{Hs}$ & $\begin{array}{l}\mathrm{K}_{\mathrm{D}}(\mathrm{nM}) \\
(\mathrm{pH} \mathrm{7.5} \mathrm{\&} \\
\left.25{ }^{\circ} \mathrm{C}\right)\end{array}$ & $\begin{array}{l}\mathrm{K}_{\mathrm{D}}(\mathrm{nM})(\mathrm{pH} \mathrm{5.5,3}, 3 \\
\left.\mathrm{M} \text { urea \& 48 }{ }^{\circ} \mathrm{C}\right)\end{array}$ & $\begin{array}{l}\mathrm{T}_{\mathrm{m}}\left({ }^{\circ} \mathrm{C}\right)(\mathrm{pH} \mathrm{5.5,3} \\
\left.\mathrm{M} \text { urea \& 48 }{ }^{\circ} \mathrm{C}\right)\end{array}$ \\
\hline cAb-HuL6 & 0.7 & 10 & $75 \pm 1$ \\
cAb-HuL22 & 35 & $\mathrm{ND}$ & $49 \pm 2$ \\
cAb-HuL22-S54C/I78C & 50 & $\sim 1000$ & $66 \pm 1$ \\
cAb-HuL5 & 460 & $\mathrm{ND}$ & $66.0 \pm 0.5$ \\
cAb-HuL5G & 310 & 2500 & $77.5 \pm 0.5$ \\
I56T and D67H lysozyme & & $56 \pm 1$ \\
\hline
\end{tabular}


$\mathrm{V}_{\mathrm{H}} \mathrm{H}$ that has a high affinity for an antigen of interest but a poor stability, can be improved either by adding a new disulphide bridge between the two beta-sheets of its framework (i.e. between residues 54 and 78, IMTG numbering) [70,71] or by grafting its CDRs to another robust $\mathrm{V}_{\mathrm{H}} \mathrm{H}$ scaffold [72] (Table 1). Moreover, due to their small size, nanobodies can be used as modular building blocks for manifold constructs to create (i) multivalent (i.e. by linking two or more nanobodies recognising the same epitope), (ii) multispecific (i.e. by linking two or more nanobodies recognising different epitopes) or (iii) multifunctional (i.e. by linking one nanobody to an enzyme or toxin) entities for research, immuno-diagnosis or therapy (for a review, see Ref. [73]) (Fig. 2, lower part). As exemplified in the next sections, some of these engineering strategies have been essential to adapt the properties of nanobodies to the investigation of the mechanism of amyloid fibril formation.

\section{Nanobodies as structural probes to investigate the molecular mechanism of amyloid fibril formation}

\subsection{Human lysozyme and systemic amyloidosis}

Human lysozyme is associated with a hereditary systemic amyloidosis. So far, seven amyloidogenic variants have been identified: I56T, Y54N, F57I, W64R, D67H, F57I/T70N and T70N/W112R (Fig. 3A) [74,75]. All the patients are heterozygous, the disease being transmitted through an autosomal dominant mechanism. This disease is associated with the aggregation of one lysozyme variant into amyloid fibrils that accumulate extracellularly into several tissues and organs (including liver, kidneys, spleen, gastrointestinal tract and salivary glands) [76,77]. The age at which amyloid deposits appear, their distribution in tissues and the clinical features are very variable both within and between families [76].

Human lysozyme is a 130 amino acid muramidase (MM $\sim 14.7 \mathrm{kDa}$ ), which is widely distributed in a variety of tissues and body fluids including liver, articular cartilage, plasma, saliva, tears and milk [78]. It is constituted of two domains: the $\alpha$-domain which contains four $\alpha$-helices (named A, B, C and D) and two short 3-10 helices, and the $\beta$-domain which contains a short 3-10 helix, a $\beta$ sheet (containing 3 strands) and a long loop. The active site is located at the interface between the two domains (Fig. 3A).

The amyloidogenic I56T and $\mathrm{D} 67 \mathrm{H}$ variants and the nonamyloidogenic T70N variant have been well characterised. Their structure, stability, folding kinetics and in vitro aggregating properties have been compared to those of the wild type protein $(\mathrm{Wt}-$ $\mathrm{HuL}$ ). The three mutations are localised within the $\beta$-domain: the I56T mutation is near the active site, while the D67H and T70N mutations are localised in the loop (Fig. 3A). X-ray diffraction studies showed that the structure of the I56T variant is identical to that of the $\mathrm{Wt}-\mathrm{HuL}$ while the $\mathrm{D} 67 \mathrm{H}$ mutation disrupts three hydrogen bonds involving residues Y54, K69 and T70. The destruction of this hydrogen-bonding network results in a rearrangement of part of the $\beta$-domain: the long loop and two $\beta$ strands of the $\beta$-domain move away from each other [79] (Fig. 3A). A similar rearrangement, although of lower amplitude, is also found in the X-ray structure of the non-amyloidogenic T70N natural variant [80]. These observations therefore indicate that there is no direct correlation between the structural changes induced by the mutations in the native state of lysozyme and the propensity of the variant proteins to form amyloid fibrils.

The I56T and D67H mutations, despite their different locations and effects on the structure of the protein, decrease to a similar extent the thermostability of the lysozyme. Their $\mathrm{T}_{\mathrm{m}}$ are about $10^{\circ} \mathrm{C}$ lower relative to the wild-type protein at all the $\mathrm{pH}$ values tested (i.e. from 2.5 to 7 ) [76], while the naturally occurring non- amyloidogenic T70N variant is destabilised by only $\sim 4{ }^{\circ} \mathrm{C}[80]$. Hydrogen/deuterium (H/D) experiments analysed by mass spectrometry and nuclear magnetic resonance (NMR) show that the significant destabilisation of the I56T and D67H variants allows them to populate transiently an intermediate species under physiologically relevant conditions of temperature and $\mathrm{pH}$ (Fig. 3D). In this species, the $\beta$-domain and the C-helix (referred to as the amylotope [44]) are cooperatively unfolded while the rest of the $\alpha$ domain remains native [81,82] (Fig. 3A). Such a species is formed neither by the Wt-HuL nor by the non-amyloidogenic T70N variant under the same conditions $[80,81]$. Thus, the formation of this intermediate species under physiological relevant conditions, due to a decrease in global cooperativity (i.e. the protein does not unfold anymore as a single entity), is a characteristic feature underlying the amyloidogenicity of the I56T and D67H lysozyme variants. The formation of intermolecular interactions between the unfolded parts of the intermediate species is likely the trigger event of amyloid fibril formation (Fig. 3E).

\subsubsection{HuL specific nanobodies $c A b-H u L 5, c A b-H u L 6$ and $c A b-$ \\ HuL22: generation, characterisation and engineering}

The pioneering study of using nanobodies as structural probes to investigate amyloid fibril formation was actually carried out with human lysozyme [69]. The choice of nanobodies was dictated by their high stability. Indeed, the amyloidogenic lysozyme variants must be incubated under rather harsh denaturing conditions (i.e. in $0.1 \mathrm{M}$ citrate buffer pH 5.5 containing $3 \mathrm{M}$ urea at $48^{\circ} \mathrm{C}$ or in $0.1 \mathrm{M}$ acetate buffer $\mathrm{pH} 5.0$ at $65{ }^{\circ} \mathrm{C}$ ) to form amyloid fibrils in vitro in a reasonable time $[69,82]$; thus, in order to use antibody fragments as molecular structural and mechanistic probes they must be stable and functional under those conditions.

Three nanobodies (named cAb-HuL5, cAb-HuL6 and cAb-HuL22) have been selected by phage display from the blood of a dromedary immunised with the monomeric Wt-HuL; this protein was also used for the panning $[37,44,83]$. These nanobodies bind to three different epitopes on the surface of lysozyme (Fig. 3B) with affinities in the nanomolar range (Table 1). The structures of cAb-HuL5 and cAb-HuL6 in complex with Wt-HuL have been determined by $\mathrm{X}$-ray crystallography. The epitope of cAb-HuL5 (i.e. the residues of HuL that are in direct contact with the nanobody) consists of 14 residues of the lysozyme molecule, located primarily on the loop between the A and B helices [83]; it does not encompass any residue from the amylotope (i.e. the region involved in the cooperative local unfolding event characteristic of the I56T and D67H variants) (Fig. 3A). The epitope of cAb-HuL6 encompasses 14 residues located in the loop between the A and B helices in the $\alpha$-domain, in the long loop within the $\beta$-domain and in the $C$-helix; 11 of these 14 residues belong to the amylotope (Fig. 3A) [69]. No crystallographic data is available for cAb-HuL22. However, several results such as (i) surface plasmon resonance (SPR) competition experiments with an analogue substrate, (ii) the lower affinity for D67H compared to that for Wt-HuL, and (iii) the comparison of NMR heteronuclear single quantum coherence (HSQC) spectra of ${ }^{15} \mathrm{~N}-\mathrm{Wt}-\mathrm{HuL}$ free and in complex with cAb-HuL22 suggest that cAb-HuL22 binds into the active site of the protein through its long CDR3 loop (i.e. containing 29 residues) [44]. Thus, the epitope of cAb-HuL22 likely encompasses some residues from the amylotope (Fig. 3A).

cAb-HuL6 is more thermostable that both the I56T and $\mathrm{D} 67 \mathrm{H}$ variants and can bind them under the conditions used to form fibrils in vitro (e.g. $0.1 \mathrm{M}$ citrate buffer $\mathrm{pH} 5.5$ containing $3 \mathrm{M}$ urea at $48{ }^{\circ} \mathrm{C}$ ) $[69,82,83]$ (Table 1 ). On the contrary, cAb-HuL22 is less thermostable than the lysozyme variants and could therefore not be used to inhibit fibril formation [44]. Thus, in order to increase the stability of this nanobody, one of the strategies shown to efficiently improve nanobodies stability was used: a disulphide bond 

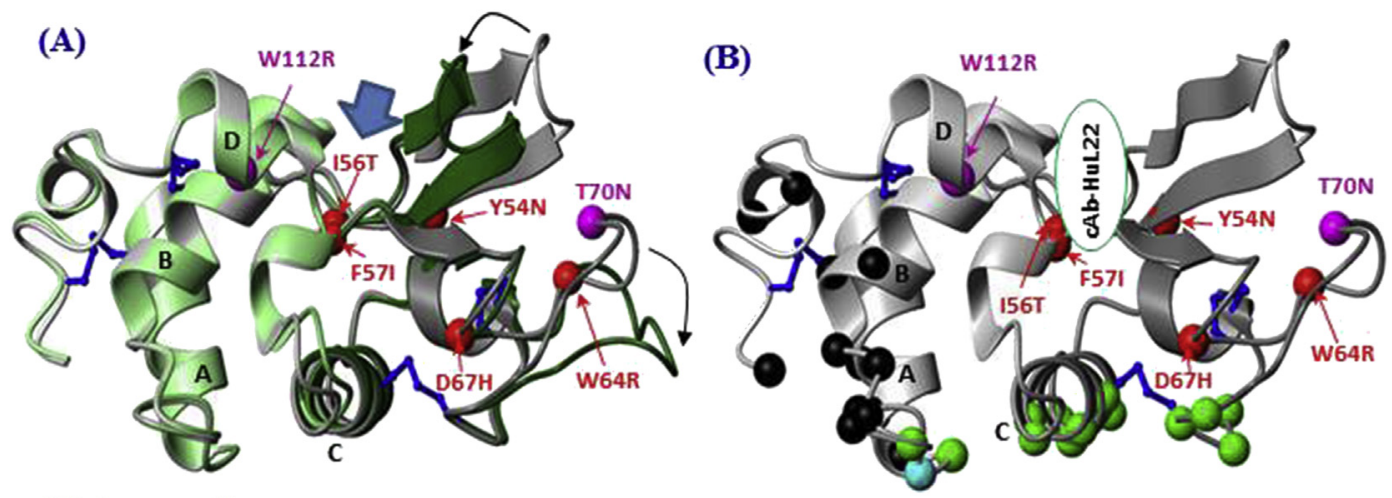

(C) Aggregation
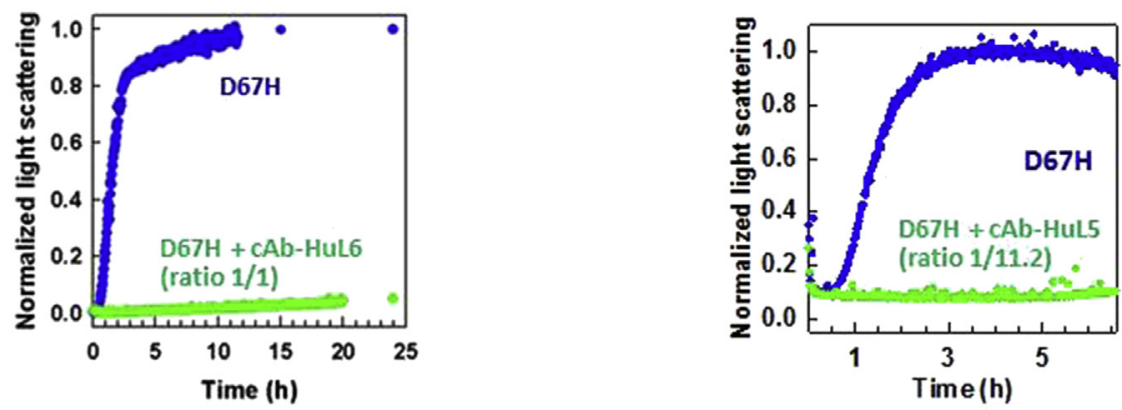

(D) H/D exchange analysed by mass spectrometry
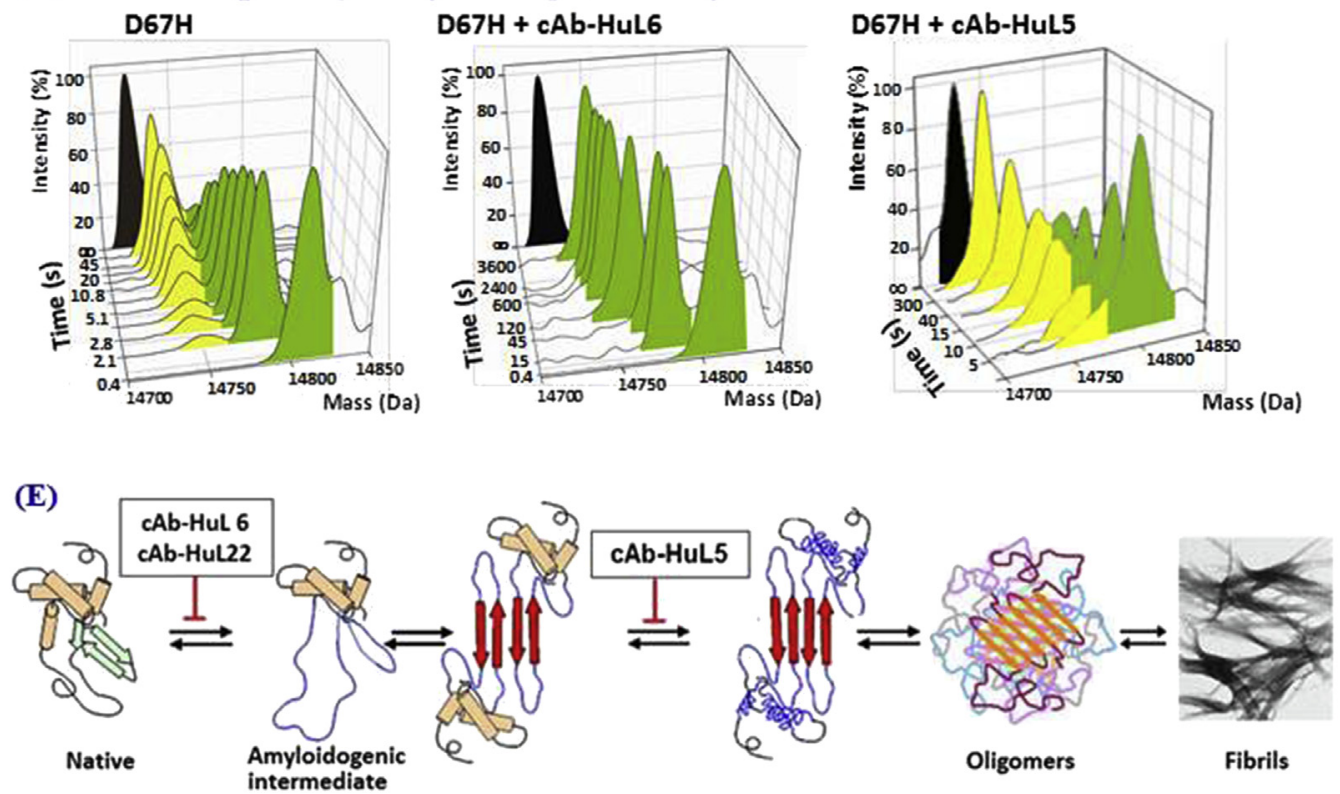

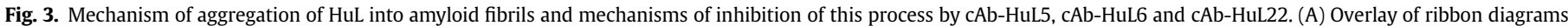

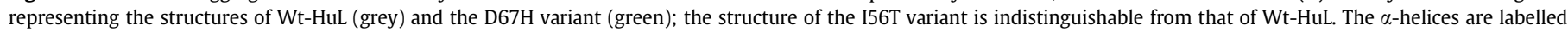

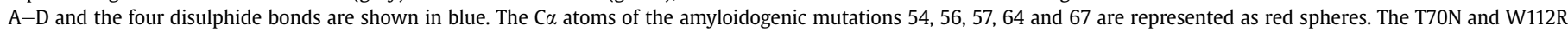

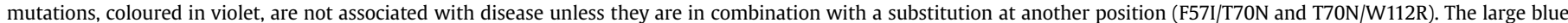

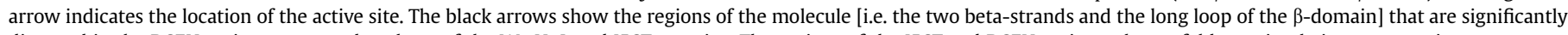

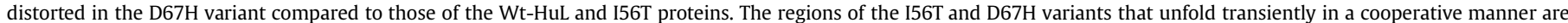

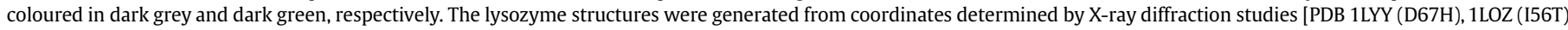

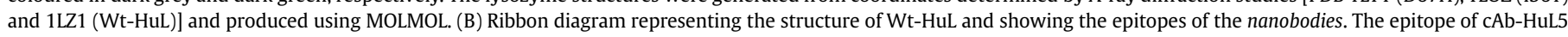

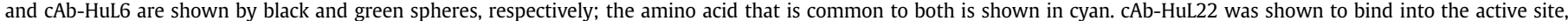

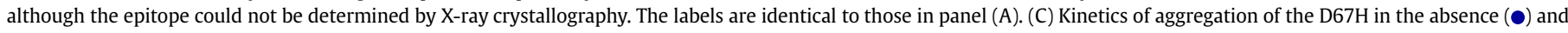

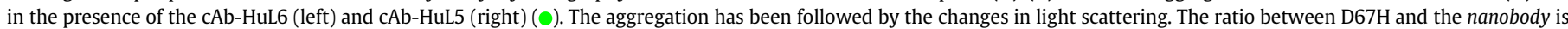

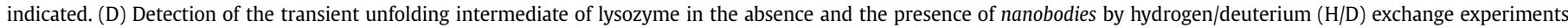

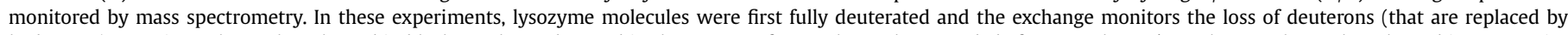

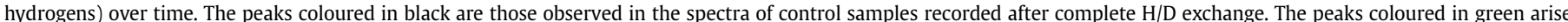

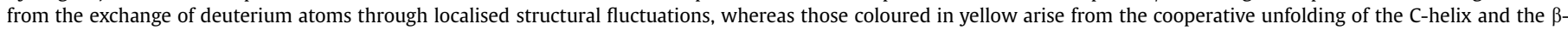

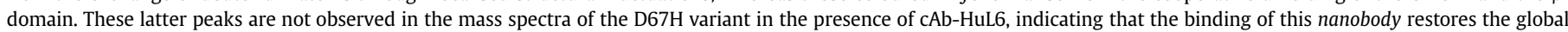

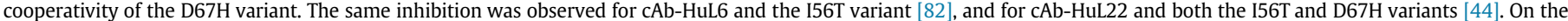

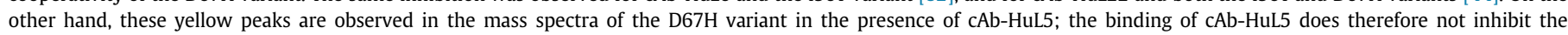


was added between the two $\beta$-sheets thanks to the S54C and I78C mutations (IMGT numbering) [70,71]. The variant obtained (named cAb-HuL22-S54C/I78C) is significantly more thermostable (i.e. its $\mathrm{T}_{\mathrm{m}}$ is $17{ }^{\circ} \mathrm{C}$ higher than that of cAb-HuL22) and its affinity for WtHuL, at least at $25{ }^{\circ} \mathrm{C}$ in $10 \mathrm{mM}$ HEPES buffer $\mathrm{pH} 7.5$, is in the same order of magnitude that of cAb-HuL22 [44] (Table 1). Finally, although cAb-HuL5 is more thermostable than I56T and D67H lysozyme variants, it significantly aggregates under the conditions used to trigger the aggregation of the latter [83]. A less aggregationprone version of cAb-HuL5 was therefore engineered by grafting its CDRs onto the extremely stable scaffold of cAb-HuL6; the chimeric nanobody obtained is referred to as cAb-HuL5G. cAb-HuL5G is more stable than cAb-HuL5: its $\mathrm{T}_{\mathrm{m}}$, in $0.1 \mathrm{M}$ citrate buffer $\mathrm{pH} 5.5$ containing $3 \mathrm{M}$ urea, is increased by $\sim 9.5^{\circ} \mathrm{C}$ (i.e. $75.5^{\circ} \mathrm{C}$ versus $66.0^{\circ} \mathrm{C}$ ) (Table 1 ). Moreover, the binding parameters of cAb-HuL5G to WtHuL are similar to those measured for cAb-HuL5 at $\mathrm{pH} 7.5$ and $25{ }^{\circ} \mathrm{C}$ (Table 1 ).

These examples highlight the main advantage of nanobodies, compared to conventional antibody fragments, to investigate the mechanism of amyloid fibril formation (Fig. 2). Indeed, their stability and solubility can be straightforwardly and easily improved by protein engineering techniques, without impairing their binding properties, to make them functional under the relatively harsh conditions that are often used to trigger in vitro aggregation of protein over a reasonable timeframe.

\subsection{2. $c A b-H u L 5, c A b-H u L 6$ and $c A b-H u L 22$ as mechanistic probes} 3.1.2.1. The binding of the three nanobodies inhibits the amyloid fibril formation of human lysozyme variants. In the presence of an equimolar amount of cAb-HuL6, the D67H (Fig. 3C) and I56T [69,82] variants of lysozyme do not significantly aggregate, even after $24 \mathrm{~h}$ of incubation, while both variants completely aggregate into amyloid fibrils in less than $5 \mathrm{~h}$ when incubated in the absence of nanobodies [69,82]. The binding of cAb-HuL22-S54C/I78C and cAbHuL5G to D67H also significantly inhibits fibril formation although, due to their low affinity under the conditions used to trigger lysozyme fibril formation, a high nanobody/lysozyme ratio (i.e. 5/1 for cAb-HuL22-S54C/I78C [44] and 11.2/1 for cAb-HuL5G [83]) is needed (Fig. 3C).

3.1.2.2. cAb-HuL5 inhibits human lysozyme fibril formation through a mechanism different from that of cAb-HuL6 and cAb-HuL22. $\mathrm{H} / \mathrm{D}$ exchange experiments analysed by mass spectrometry have shown that the binding of cAb-HuL6 (Fig. 3D) and cAb-HuL22 [44] prevents the formation of the intermediate species by both the D67H (Fig. 3D) and I56T variants [82]. These results suggest that these nanobodies inhibit the fibril formation by restoring the global cooperativity of the variants that is characteristic of the wild-type lysozyme. Moreover, they further support the fact that the formation of the partially unfolded lysozyme intermediate triggers the formation of amyloid fibrils. The crystal structure of the cAb-HuL6/ Wt-HuL complex indicates that only 11 residues of the nearly 60 involved in the transient cooperative unfolding of the I56T and $\mathrm{D} 67 \mathrm{H}$ variants are in direct contact with the nanobody. Thus, the effects of the binding of cAb-HuL6 is not just to mask the entire region that is destabilised by the mutation and hence to prevent the unfolding from the rest of the structure [69]. Comparison of the
NMR HSQC spectra of the lysozyme variants alone and in complex with the nanobodies has allowed the long-range effects of nanobody binding to be mapped. Note that the small mass of the nanobodies and thus the limited mass of the complex prevents an excessive loss of the NMR signal and thus allows identifying peaks whose chemical shift is significantly perturbed upon the binding of the nanobody. This analysis shows that $\sim 30$ residues of lysozyme exhibit a significant change in chemical shift upon binding of cAbHuL6, most of them are located in positions directly in contact with the nanobody or in close proximity to the latter. A few affected residues are however far from the binding site and reflect a long range effect through the backbone. They include residues 56 and 57 which are locations of the I56T and F57I amyloidogenic mutations and that are essential for the integrity of the interface between the $\alpha$ - and $\beta$-domains the protein $[69,82]$. It was therefore proposed that the binding of cAb-HuL6 to the amyloidogenic variants restores the global cooperativity characteristic of the wild-type protein through, at least in part, long-range subtle conformational effects which stabilise the interface between the two structural domains of the protein. The same mechanism of action was proposed for $\mathrm{CAb}-$ HuL22 that binds into the active site of the protein [44].

By contrast, the binding of cAb-HuL5 does not prevent the formation of the intermediate species by both the D67H (Fig. 3D) and I56T variants [83]; it does not, therefore, restore the global cooperativity of the lysozyme variants. The effects of the binding of a nanobody on the properties of the interface between the $\alpha$ - and $\beta$ domains, and thus on the global cooperativity of the amyloidogenic lysozyme variants, are therefore highly dependent on the epitope. Moreover, these results suggest that this cAb-HuL5 could act at a later stage on the process of fibril formation (Fig. 3E). cAb-HuL5 does not bind to human lysozyme in its fibrillar form, indicating that its epitope does not maintain its native state in the aggregated form of lysozyme. Thus, cAb-HuL5, which binds to the $\alpha$-domain of the lysozyme, is thought to inhibit the formation of amyloid fibrils by preventing the unfolding of the $\alpha$-domain, the latter event is therefore a crucial step in the formation of amyloid fibrils by human lysozyme [83].

\subsubsection{Conclusions}

The results obtained with cAb-HuL5, cAb-HuL6 and cAb-HuL22 indicate that nanobodies can inhibit the formation of amyloid fibrils at different stages of the process of amyloid fibril formation. This pioneering work has demonstrated the value of nanobodies as structural probes to study the molecular mechanisms of amyloid fibril formation.

\section{2. $\beta 2-$ Microglobulin and dialysis-related amyloidosis}

The dialysis-related amyloidosis (DRA) is associated, at least in part, with the increase in $\beta 2$-microglobulin $(\beta 2 \mathrm{~m})$ concentration (up to 60 -fold) during long-term haemodialysis, which leads to the aggregation of $\beta 2 \mathrm{~m}$ into insoluble amyloid fibrils that accumulate extracellularly in bones and joints [84,90,91]. This deposition causes painful arthropathy, cysts and pathologic fracture. Up to $\sim 30 \%$ of the $\beta 2 \mathrm{~m}$ found in these aggregates are actually a truncated form lacking the six $\mathrm{N}$-terminal amino acids $(\Delta \mathrm{N} 6 \beta 2 \mathrm{~m})[85,86]$. Recently, another form of $\beta 2 \mathrm{~m}$ amyloidosis was discovered, in

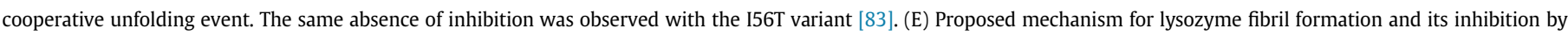

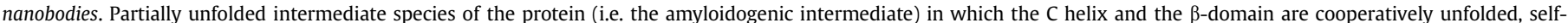

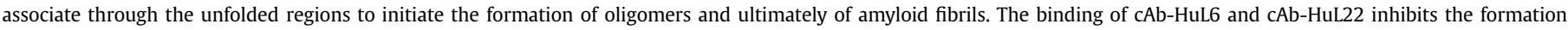

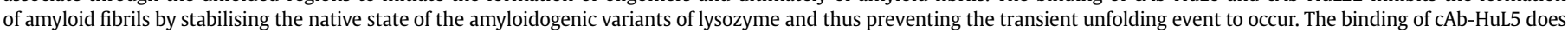

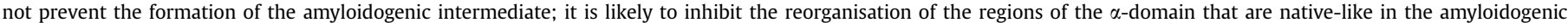

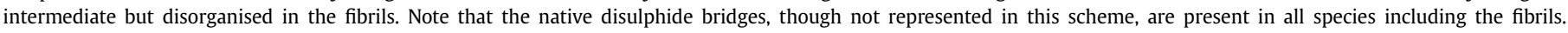
Figure adapted from Refs. [44,69,83]. 
which the full length form of the naturally occurring D76N variant of $\beta 2 \mathrm{~m}$ aggregates into amyloid fibrils whose deposition is not restricted to musculo-skeletal system but also occurs in viscera (i.e. in spleen, liver, heart and salivary glands) and nerves, mainly around endoneurial capillaries and within vessel walls [84]. Contrary to DRA, the patients suffering from this hereditary systemic amyloidosis have normal renal function and a normal circulating $\beta 2 \mathrm{~m}$ concentration [84]. The D67N pathogenic variant, unlike the wild-type protein, has a very high propensity to form amyloid fibrils in vitro under physiologically relevant $\mathrm{pH}$ and temperature conditions.

$\beta 2 \mathrm{~m}$ is a 130 amino acid protein ( $\mathrm{MM} \sim 11.7 \mathrm{kDa}$ ) corresponding to the light chain of the type I major histocompatibility complex (MHC-1) [87]. The MHC-I molecules are responsible for antigen presentation in a process controlling the immune response against "self" and "non-self" antigens. $\beta 2 \mathrm{~m}$ is expressed on the surface of nearly all nucleated cells and it adopts an immunoglobulin fold, made up of a seven-stranded $\beta$-sandwich linked by a disulphide bridge (Fig. 4E, left part) $[88,89]$. Although the increased concentration of $\beta 2 \mathrm{~m}$ is a decisive factor in causing DRA, it is not sufficient by itself. The nature of the other factor(s) that trigger(s) the formation of $\beta 2 \mathrm{~m}$ amyloid fibril is still a matter of intense debate. They include the age of the patient, duration of renal dysfunction, interactions with a range of molecules (e.g. copper ions, glycosaminoglycans and collagen), and the formation of one or more amyloidogenic intermediates $[90,91]$. A $\beta 2 \mathrm{~m}$ conformer with a nonnative peptidyl-Pro32 trans peptide bond is, in particular, thought to serve as a direct precursor of dimeric species and oligomers that accumulate in the early phase of fibril formation [92]. The exposure of Trp60, which is located in the DE loop, upon dissociation of $\beta 2 \mathrm{~m}$ from the MHC-1 complex may also play a critical role in promoting fibril formation [93].

\subsubsection{Generation and characterisation of $\beta 2 \mathrm{~m}$ specific nanobodies}

A series of eight nanobodies has been selected by phage display from the blood of camel and llama immunised with the monomeric human Wt- $\beta 2 \mathrm{~m}$ or $\Delta \mathrm{N} 6 \beta 2 \mathrm{~m}$; these two proteins were also used for the panning [94]. The affinities of these nanobodies for $\beta 2 \mathrm{~m}$ and its $\Delta \mathrm{N} 6$ variant are in the nanomolar range (Fig. 4D).

\subsubsection{Nanobodies as mechanistic probes: inhibition of $\beta 2 m$ amyloid fibril formation}

The ability of nanobodies to inhibit fibril formation was investigated using $\Delta \mathrm{N} 6 \beta 2 \mathrm{~m}$, since this protein has a greater in vitro propensity to form amyloid fibrils than the full length $\beta 2 \mathrm{~m}$ [95]. When incubated at $\mathrm{pH} 5,37^{\circ} \mathrm{C}$, in the presence of salt, $\Delta \mathrm{N} 6 \beta 2 \mathrm{~m}$ is rapidly (i.e. within $124 \mathrm{~h}$ ) converted into 'non-amorphous aggregates' that bind thioflavin-T(ThT); after 2 weeks of incubation, the first amyloid fibrils start to grow out of these aggregates while all aggregates are converted into amyloid fibrils after 4 weeks [94]. Remarkably, in the presence of a $12 \%$ excess of five of the eight nanobodies, $\Delta \mathrm{N} 6 \beta 2 \mathrm{~m}$, incubated at $37^{\circ} \mathrm{C}$, does not aggregate within $124 \mathrm{~h}$ (Fig. $4 \mathrm{~A}-\mathrm{C}$ ). One of the nanobodies, $\mathrm{Nb24}$, when added in an equimolar amount, can even disrupt preformed $\Delta \mathrm{N} 6 \beta 2 \mathrm{~m}$ 'non-amorphous aggregates', leading to an increased soluble fraction of the truncated protein. $\mathrm{Nb} 24$ is, however, unable to dissociate preformed $\Delta \mathrm{N} 6 \beta 2 \mathrm{~m}$ amyloid fibrils.

\subsubsection{Nanobodies as structural probes: structure of the nucleus for $\beta 2 m$ fibrillogenesis}

The five inhibitory nanobodies were tested as co-crystallisation chaperones of $\triangle \mathrm{N} 6 \beta 2 \mathrm{~m}$ but diffracting crystals were obtained only for the complex with $\mathrm{Nb24}$ [94]. Under the conditions of crystallisation of the complex, the $\Delta \mathrm{N} 6 \beta 2 \mathrm{~m}$ alone aggregates within minutes, indicating that the nanobody indeed serves as an efficient crystallisation chaperone. The crystal structure obtained at high resolution $(2.2 \AA)$ reveals that the $\Delta \mathrm{N} 6 \beta 2 \mathrm{~m}$ variant forms a domain-swapped dimer exchanging residues 91-94 which forms the G-strand. This G-strand swap is accompanied by the extension of the hinge loop of each monomer which interact to each other to form of a new $\beta$-sheet composed of two anti-parallel $\beta$-strands (Fig. 4E, right part). The extended hinge loop corresponds to the heptapeptide NHVTLSQ (residues 83-89) and very interestingly, the polypeptide NHVTLS has been found to have an intrinsic high tendency to form short fibrils in vitro [96]. Moreover, in the $\triangle \mathrm{N} 6 \beta 2 \mathrm{~m}$ domain-swapped dimer, the backbone $\mathrm{NH}$ and $\mathrm{CO}$ groups of V85 and L87 are exposed to the solvent, and therefore are prone to interact with $\beta$-strands of other swapped dimers or opened monomers (i.e. with the extended hinge loop). In addition, while in $\beta 2 \mathrm{~m}$ monomer Pro32 adopts the cis conformation, it adopts a trans conformation into the domain-swapped dimer; as mentioned above, this non-native peptidyl-Pro32 trans peptide bond is thought to be a critical event in the initiation of the aggregation process. Altogether, these observations suggest that the domainswapped dimer could act as a plausible nucleus for $\beta 2 \mathrm{~m}$ fibrillogenesis, which can recruit other swapped dimers or opened monomers by a self-templated mechanism. The binding of $\mathrm{Nb} 24$ could prevent the aggregation of $\beta 2 \mathrm{~m}$ by steric hindrance, by mass action or by prohibiting potential structural changes in the core of $\beta 2 \mathrm{~m}$ (Fig. 4F).

$\mathrm{Nb} 24$ was also used as crystallisation aid to solve the structure of P32G $\beta 2 \mathrm{~m}$, a non-natural variant of $\beta 2 \mathrm{~m}$ that has a higher propensity than the wild-type protein to form amyloid fibrils in vitro [97]. In this case, $\beta 2 \mathrm{~m}$ crystallises as a monomer in complex with $\mathrm{Nb} 24$. Like in the swapped dimer formed by $\Delta \mathrm{N} 6 \beta 2 \mathrm{~m}$, the peptide bond at position 32 also adopts a trans conformation in P32G $\beta 2 \mathrm{~m}$ in complex with Nb24 while Pro32 adopts a cis conformation in the wild-type protein. The trans conformation in position 32 in P32G $\beta 2 \mathrm{~m}$ causes several structural rearrangements that may increase the susceptibility of the protein to aggregate into amyloid fibrils [97]. In particular, the $\beta$-strand D is less kinked in P32G $\beta 2 \mathrm{~m}$ than in wild-type $\beta 2 \mathrm{~m}$ and is therefore more prone to intermolecular pairing. It also leads to the rearrangement of several hydrophobic residues and to a concomitant increase in the surface hydrophobicity that could also contribute the increased propensity of P32G $\beta 2 \mathrm{~m}$ to form amyloid fibrils [97].

\subsubsection{Detection of the $\beta 2 \mathrm{~m}$ in the MHC-I at the surface of cells}

$\mathrm{Nb} 24$ does not bind to $\beta 2 \mathrm{~m}$ within the MHC-I complex exposed on the surface of human cells [94]. Thus, this nanobody efficiently blocks the formation of amyloid fibrils without interfering with the biological function of $\beta 2 \mathrm{~m}$. This suggests that $\mathrm{Nb} 24$ could be developed as a therapeutic tool to prevent amyloid deposits in dialysis patients; it remains however to demonstrate that the species stabilised by $\mathrm{Nb} 24$ are not toxic.

\subsubsection{Conclusions}

Altogether, these two studies involving Nb24 and two variants of $\beta 2 \mathrm{~m}$ supports that the Pro32 cis to trans switch is determinant for the aggregation of $\beta 2 \mathrm{~m}$ into amyloid fibrils [97]. Moreover, they demonstrate that nanobodies can be used as chaperones to trap transient amyloidogenic intermediate species and allow their structural characterisation at atomic resolution

\subsection{Human $\alpha$-synuclein and Parkinson's disease}

Parkinson's disease (PD), familial or sporadic, is the second most common age-related neurodegenerative disorder, affecting about $1 \%$ of the population over the age of 70 [98]. It is characterised by tremor, slowness of movements, muscular rigidity and impairment of 


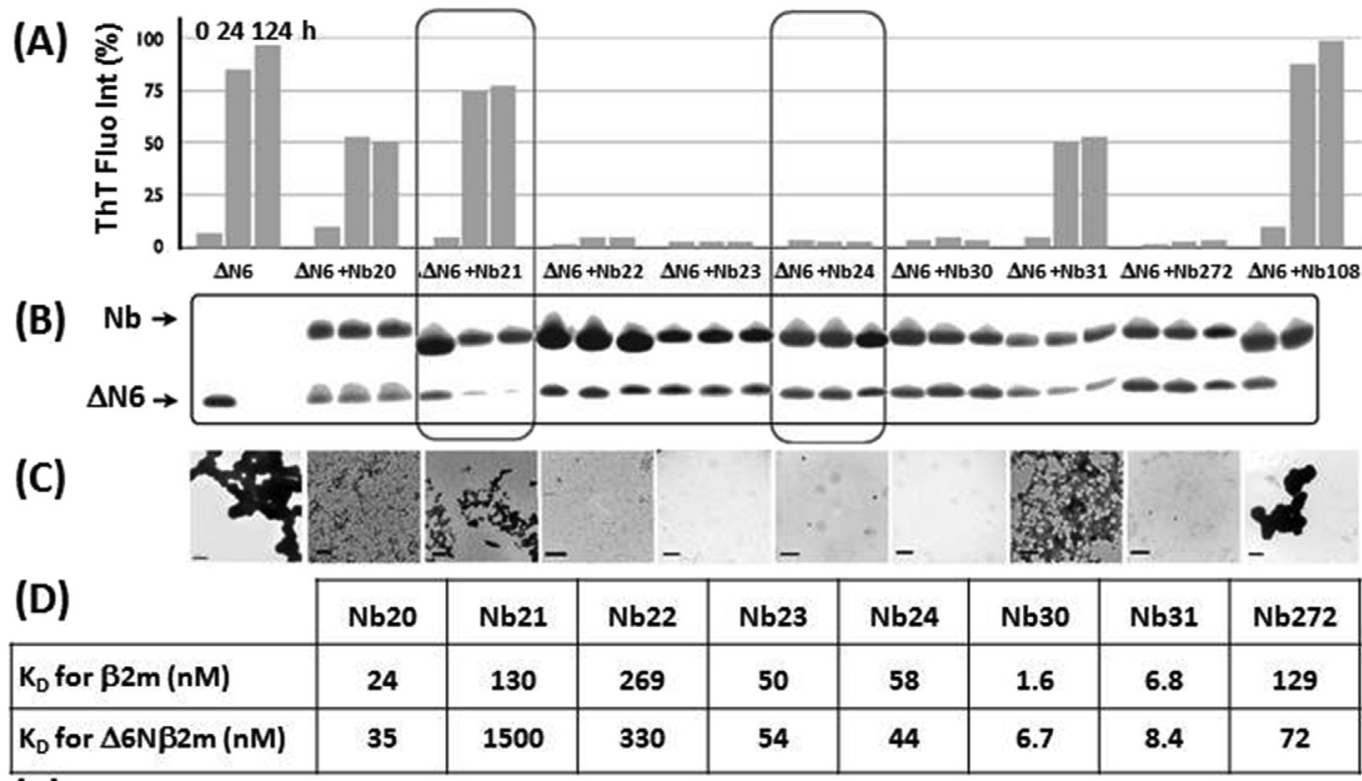

(E)

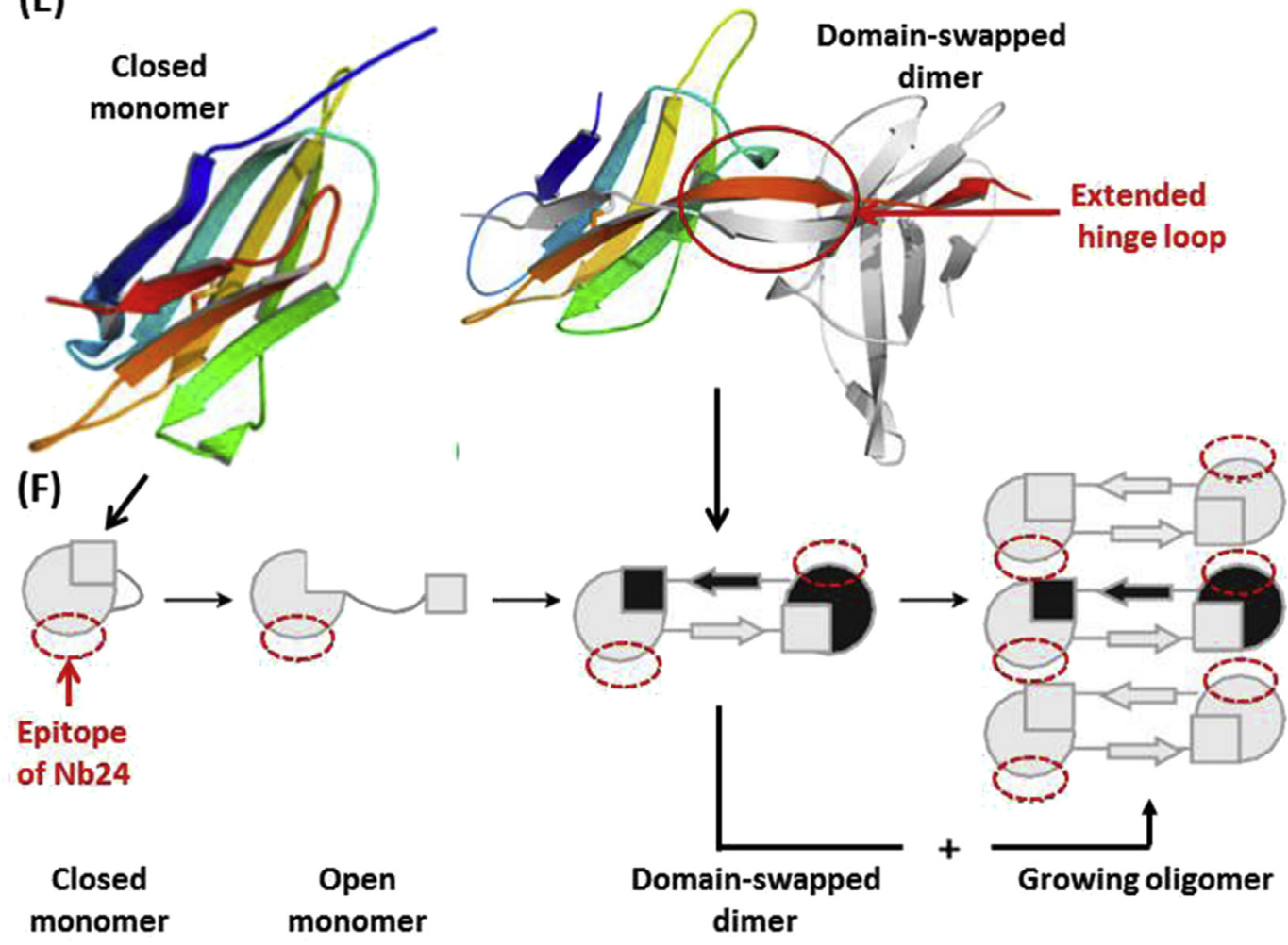

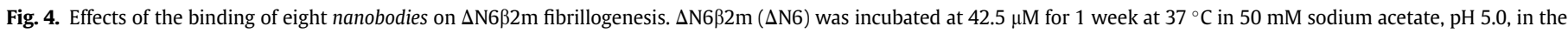

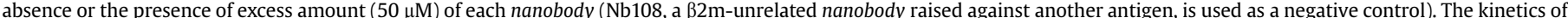

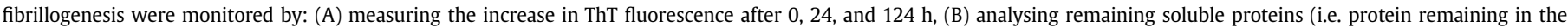

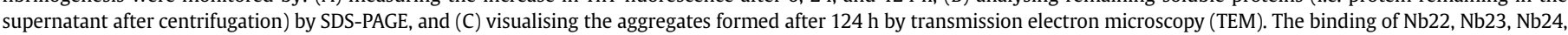

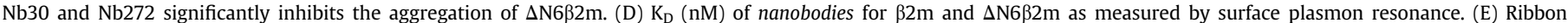

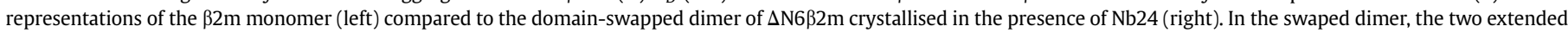

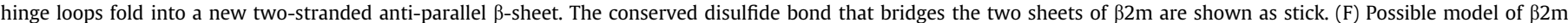

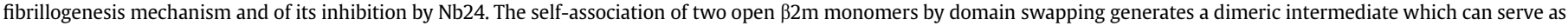

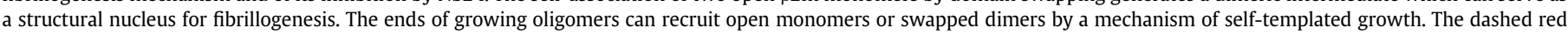

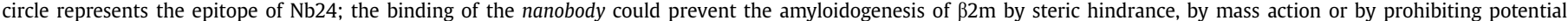
structural changes in the core of $\beta 2 \mathrm{~m}$, thus preventing the elongation of oligomers. Figure adapted from Ref. [94].

postural reflexes [98]. One of the pathological hallmarks of PD is the progressive loss of dopaminergic neurons in the substantia nigra pars compacta of the brain, associated with the formation of intracellular fibrillar inclusions known as Lewy bodies (LB) and Lewy neurites. These inclusions are essentially made of $\alpha$-synuclein ( $\alpha$ Syn) [99].

aSyn is a relatively small (140 aa) and soluble intracellular protein which is especially abundant in neural tissues [100]. The 
normal cellular function of $\alpha$ Syn is not clearly determined yet [101]; it has however been suggested that it has a role in the regulation of synaptic function, plasticity and neurotransmission release [102]. Several studies have indicated that $\alpha$ Syn may also act as chaperone for the formation of presynaptic SNARE (soluble N-ethylmaleimidesensitive-factor attachment protein receptor) complexes; SNARE are transmembrane proteins that mediate the fusion of synaptic vesicles [103,104]. A number of genetic mutations, including A30P, E46K and A53T (Fig. 5A), and the triplication of the $\alpha$ Syn gene are correlated with early onset of the disease [105]. Extensive experimental data indicate that $\alpha$ Syn belongs to the family of natively unfolded proteins, characterised by a lack of typical secondary structures and thus of tightly packed tertiary structure [106]. A recent study has, however, suggested that it might form, in the cell, a dynamic tetramer that is not prone to aggregation [107]. Three regions can be distinguished in the $\alpha$ Syn sequence $[106,108]$ (Fig. 5A). (i) An N-terminal segment (residues 1-60) which is positively charged and contains imperfect repeats of 11 residues
(R1-R4), each with a high propensity to form amphipathic helices upon interaction with lipids. (ii) A central hydrophobic region known as the NAC (non-A $\beta$ component) region (residues 61-95) which is believed to be responsible for the formation of fibrils. Mutation of only one residue in the NAC region significantly perturbs the aggregating properties of the protein [109]. And (iii) a polar and negatively charged C-terminal region (residues 96-140) which also contains 5 prolines. The presence of negatively charged residues and prolines at the C-terminus suggests that this region has a tendency to remain in disordered conformation. This domain is, however, thought to regulate the aggregation process by shielding the NAC region [110].

Under aggregation-prone conditions, the monomeric protein changes from either $\alpha$-helical lipid-bound, helically folded tetramer or intrinsically disordered conformation towards highly ordered $\beta$ sheet fibrillar structure. As for other diseases, increasing evidence suggests that the oligomeric precursors and pre-fibrillar species, owing to their ability to interact with membranes, are primarily
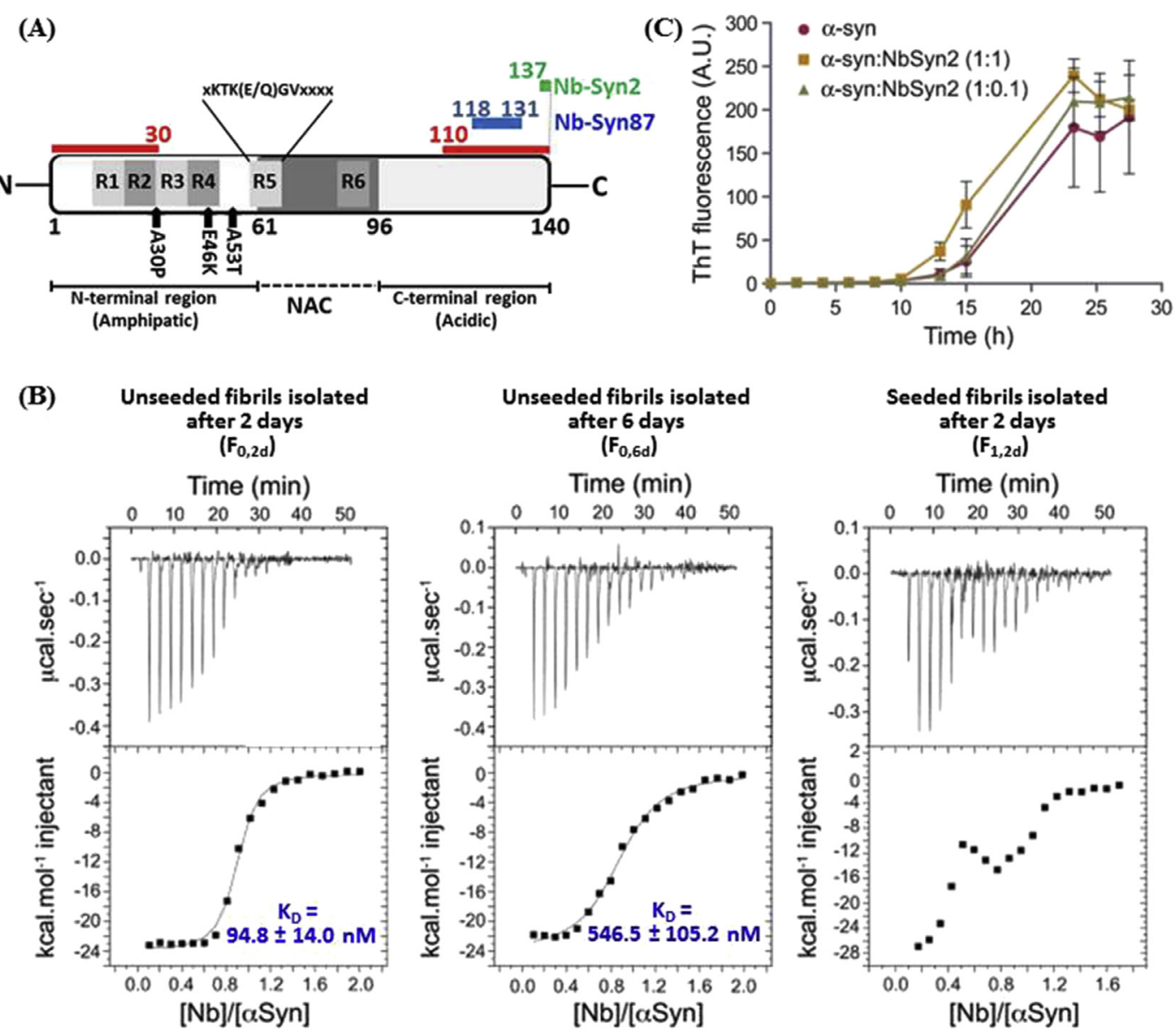

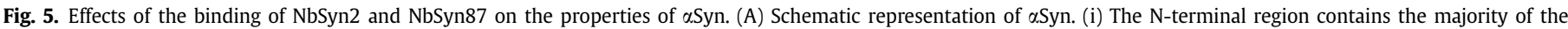

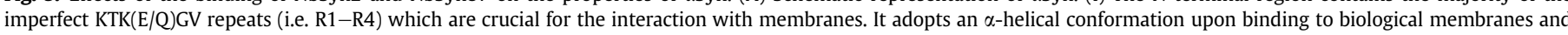

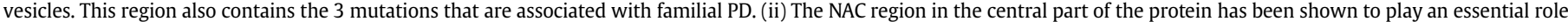

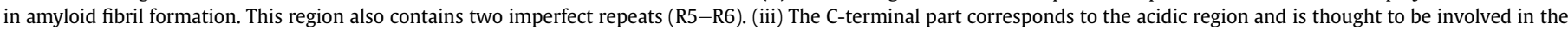

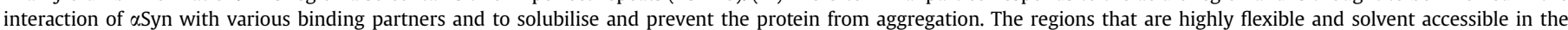

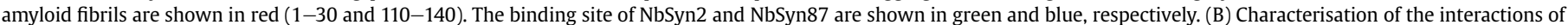

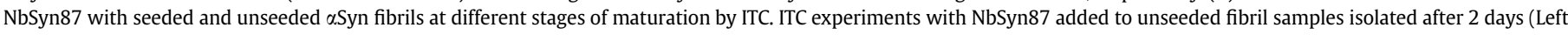

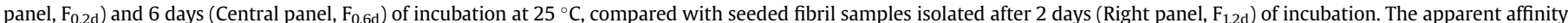

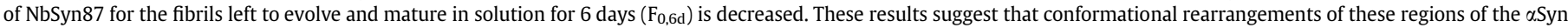

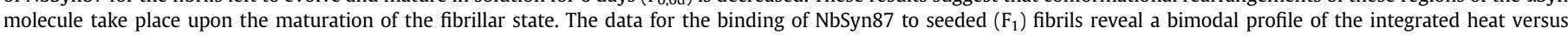

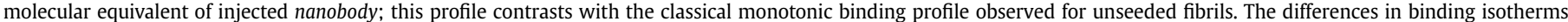

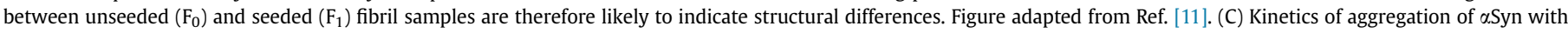

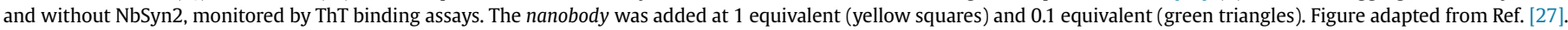


responsible for cytotoxicity and neuronal cell death, rather than the mature fibrils [111,112]. Several studies have shown that amyloid fibrils of $\alpha$ Syn consist of intertwined protofilaments, each consisting of a five-stranded $\beta$-sheet, leaving residues $1-30$ and $110-140$ highly flexible and solvent accessible (Fig. 5A). Although these Nand C-terminal regions are not directly involved in the assembly of the protofilament core, and do not adopt a $\beta$-sheet conformation, they play a key role in the interactions between the protofilaments, which is necessary for their stabilisation and fibril maturation [113].

\subsection{1. $\alpha$ Syn specific nanobodies NbSyn2 and NbSyn87: generation and characterisation}

Two nanobodies, specific to $\alpha$ Syn, have been isolated by phage display from the blood of dromedary [27] and llama [11] immunised with the monomeric human Wt- $\alpha$ Syn or the natural amyloidogenic variant A53T- $\alpha$ Syn. These nanobodies are named NbSyn 2 and NbSyn87, and they both bind to the soluble form of $\alpha$ synuclein in a $1: 1$ binding stoichiometry with nanomolar affinity (i.e. $K_{D}=14.2 \pm 8.4$ and $129.8 \pm 22.7 \mathrm{nM}$ for NbSyn87 and NbSyn2, respectively). Circular dichroism experiments show that the binding of both nanobodies does not induce significant changes in the secondary structure of $\alpha$ Syn. Their epitopes were mapped by NMR, comparing the HSQC spectra of the ${ }^{15} \mathrm{~N}-\alpha$ Syn alone and in the presence of increasing concentrations of the unlabelled nanobodies. Importantly, as for lysozyme, thanks to the small size of the nanobodies, the complex has a limited mass that prevents an excessive loss of the NMR signal and thus allows the epitope to be straightfowardly mapped. The titration of $\alpha$ Syn by both nanobodies results in either broadening or chemical shift perturbations of resonances; the amino acids that display the most significantly perturbed resonances are 118-137 and 130-140 for NbSyn87 and NbSyn2, respectively, both belonging to the C-terminal region of aSyn (Fig. 5A). The study of the interaction of NbSyn87 with a series of peptides spanning the $\alpha$ Syn sequence from residues 118 to 140 further indicates that the majority of the epitope recognised by NbSyn 87 is located between residues 118 and 131, and that residues 118 and 119 are critical for binding [11]. The crystal structure of NbSyn2, bound to a peptide corresponding to the last 9 residues of $\alpha$ Syn, reveals that only the last four amino acids of aSyn (137-140) are in direct contact with the nanobody forming a linear epitope [27].

\subsubsection{NbSyn2 and NbSyn87 as structural probes}

Isothermal titration calorimetry (ITC) and quartz crystal microbalance (QCM) data indicate that both nanobodies can bind, even simultaneously, to the aSyn amyloid fibrils, indicating that their specific epitopes, encompassing the C-terminal domain of $\alpha$ Syn, are exposed to the solvent in the fibrils [11,27]. An NMR experiment, in which increasing concentrations of ${ }^{15} \mathrm{~N}$-labelled $\mathrm{NbSyn} 2$ were added to the suspension of $\alpha$-Syn amyloid fibrils, shows that, one average, four molecule of $\alpha$ Syn in the fibril interact with only one molecule of NbSyn2. This ratio could be explained by the steric hindrance of NbSyn2, i.e. one molecule of NbSyn2 could prevent the binding of another molecule of NbSyn 2 on the nearest $\alpha$-synuclein C-termini [27]. Another possibility is the fact that the majority of C-terminus residues of $\alpha$-synuclein are buried within the fibrils. This latter possibility is supported by the structure of fibrils, in which only one in four termini is accessible to the solvent [27].

ITC experiments carried out with unseeded fibrils (i.e., fibrils formed in the absence of seeds) obtained after 2 days $\left(F_{0,2 d}\right)$ show that the apparent affinities of both nanobodies are lower than those measured for the soluble monomer of $\alpha$ Syn (Fig. 5B). Very interestingly, the apparent affinity of both nanobodies for the same fibrils left to evolve and mature in solution for 6 days $\left(F_{0,6 \mathrm{~d}}\right)$ is further decreased (Fig. 5B). Moreover, the profile of the integrated heat versus molecular equivalent of injected nanobody for the binding of both $\mathrm{Nb} 2$ and $\mathrm{NbSyn} 87$ to seeded fibrils $\left(\mathrm{F}_{1}\right.$, i.e. fibrils formed in the presence of seeds) is bimodal (Fig. 5B) whereas a classical monotonic binding profile was observed for the binding to unseeded fibrils $\left(F_{0}\right)$. The different binding isotherms between unseeded and seeded fibrils are therefore likely to indicate structural differences between the two types of fibrils. Therefore, these results suggest that conformational rearrangements of the $\mathrm{C}$-terminal region of the aSyn molecule take place upon the maturation of the fibrillar state. No differences between $F_{0,2 d}, F_{0,6 d}$ and $F_{1}$ fibrils were observed by other techniques such as ThT binding or TEM indicating that nanobodies are able to target forms of potentially pathogenic aggregates that differ from each other by relatively minor details of their structures [11].

\subsubsection{Nb2Syn as a mechanistic probe}

NbSyn2 does not significantly influence the kinetics of $\alpha$ Syn amyloid fibril formation (Fig. 5C) suggesting that the last four Cterminal residues of aSyn (137-140) do not play a part in the structure or stability of any intermediate species whose formation affects the aggregation kinetics [27]. At an equimolar concentration of NbSyn2/ $\alpha$ Syn, the kinetics of amyloid fibril formation, probed by ThT fluorescence, exhibits however a shorter lag-phase $(\sim 2.5 \mathrm{~h})$ (Fig. 5C) and a higher number of shorter fibrils is observed. Thus, in the presence of the nanobody, the rate of fibril fragmentation seems higher, explaining the shorter lag phase in the aggregation reaction probed by ThT fluorescence.

\subsubsection{Conclusions}

Altogether, these studies demonstrate that nanobodies can act as powerful molecular probes to gain insights into the structure of amyloid fibrils and probe relatively minor structural changes upon their maturation that cannot be observed by other techniques. Moreover, since it does not interfere with fibril formation, NbSyn2 can be used as a silent structural probe to investigate the exposure of C-termini in various species along the pathway of fibril formation and in mature fibrils. This silent probe could allow the study of the influence of the environment on the pathway of fibril formation through the structural rearrangement of the $\alpha$ Syn C-terminus region in the different species.

\subsection{Prion and prion diseases}

Prion diseases, also known as transmissible spongiform encephalopathies (TSEs), are a family of progressive transmissible neurodegenerative diseases that affect humans and animals. The major neuropathological hallmarks of TSEs are extensive spongiosis, neuronal loss in the central nervous system, gliosis and deposition of amyloid fibrils [114]. TSEs are a family of diseases with a wide variety of clinical manifestations including psychiatric symptoms such as agitation, behavioural disturbance, hallucination and difficulty walking and speaking $[115,116]$. TSEs are associated with the conversion of the normal cellular prion protein $\left(\operatorname{PrP}^{\mathrm{C}}\right)$ into $\beta$-sheet-rich scrapie conformers ( $\mathrm{PrP}^{\mathrm{Sc}}$ ) which aggregate into amyloid fibrils and deposit extracellularly, leading to neuronal death and spongiform changes in the brain parenchyma. The most common human prion disease form is the sporadic Creutzfeldt-Jakob disease with one or two new patients per million and per year worldwide [117]; moreover, more than 40 mutations in the prion protein lead to hereditary autosomal dominant form of the disease [117]. The disease can also be transmitted to humans by eating diseased meat, by receiving corneal transplants from infected donors and through contaminated medical equipment [118]. According to the protein-only-hypothesis, $\mathrm{PrP}^{\mathrm{Sc}}$ is the essential causative agent of prion disease and transmission, acting as a truly 
infectious agent [115]. Indeed, once generated, $\mathrm{PrP}^{\mathrm{Sc}}$ acts as a conformational template to promote the conversion of $\operatorname{PrP}^{C}$ into $\mathrm{PrP}^{\mathrm{Sc}}$ and to propagate the pathology from cell to cell, from tissue to tissue, and even between hosts for some TSEs [119]. Despite the fact that $\operatorname{PrP}^{C}$ and $\operatorname{PrP}^{S c}$ have the same amino acid sequence, they differ in several aspects. Unlike $\operatorname{PrP}^{C}, \operatorname{PrP}^{\mathrm{Sc}}(\mathrm{i})$ is insoluble in non-ionic detergents, (ii) is partially resistant to proteinase $\mathrm{K}$ digestion, and (iii) presents an increased content in $\beta$-sheet structure [120].

Human $\operatorname{PrP}^{\mathrm{C}}$ is a 208 amino-acid glycoprotein widely expressed in various tissues; the highest levels of $\operatorname{PrP}^{C}$ are found in neurons but it can be found in other organs including spleen, kidneys, heart and lungs [121]. Mature $\operatorname{PrP}^{\mathrm{C}}$ is found predominantly anchored by glycosyl-phosphatidylinositol to the extracellular cell surface. Although a clear-cut function for $\operatorname{PrP}^{C}$ in the central nervous system (CNS) is yet to be established, a role in memory is probable [121]. In the peripheral nerves, $\operatorname{PrP}^{\mathrm{C}}$ appears to have an important contribution in myelin maintenance. $\operatorname{PrP}^{C}$ might also be involved in numerous biological activities including cell-signalling, neurogenesis, neuroprotection and synpaptic transmission [121]. $\operatorname{PrP}^{C}$ is composed of two domains [122] (Fig. 6A): (i) a flexible disordered $\mathrm{N}$-terminal domain (residues 23-124) including several octapeptide repeats (60-91) and a positively charged segment (residues 96-111), and (ii) a structured globular C-terminus (residues 125-231) containing three $\alpha$-helices, two of them being linked by a disulphide bridge and two very short anti-parallel $\beta$-strands. This $\beta 1-\alpha 1-\beta 2-\alpha 2-\alpha 3$ anti-parallel $\beta$-ribbon structure is referred to as the $\mathrm{PrP}^{\mathrm{C}}$-like fold in the Structural Classification of Protein (SCOP) database [123]. Conversion of $\operatorname{PrP}^{C}$ into $\operatorname{PrP}^{\mathrm{Sc}}$ is associated with simultaneous loss of $\alpha$-helix content and considerable gain of $\beta$ sheet structure which correlates with the characteristic partial resistance to proteinase $\mathrm{K}$ (residues 90 to 231 become resistant to proteolysis). There is a lot of evidence indicating that important structural changes also occur in the $\mathrm{N}$-terminal region (i.e., in the region incompassing residues 90-120), and particularly in the

(A)

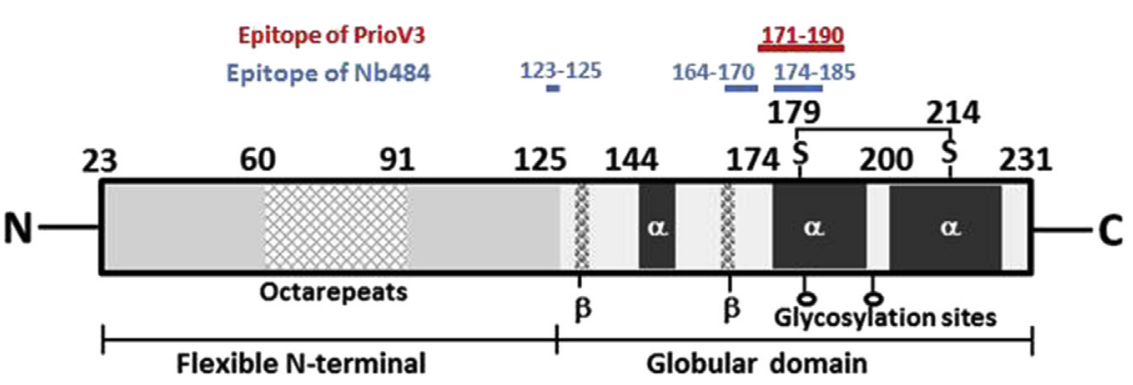

(B)

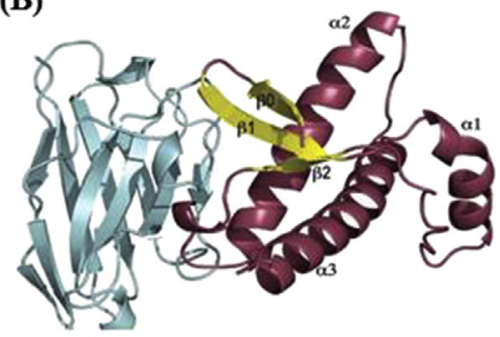

(D) $\mathrm{kDa}$

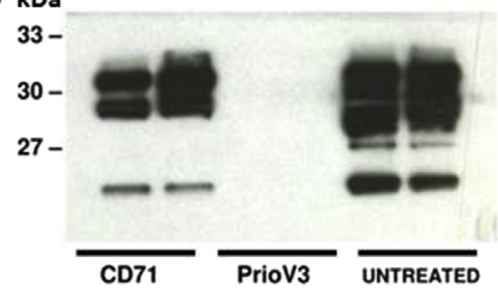

(C)

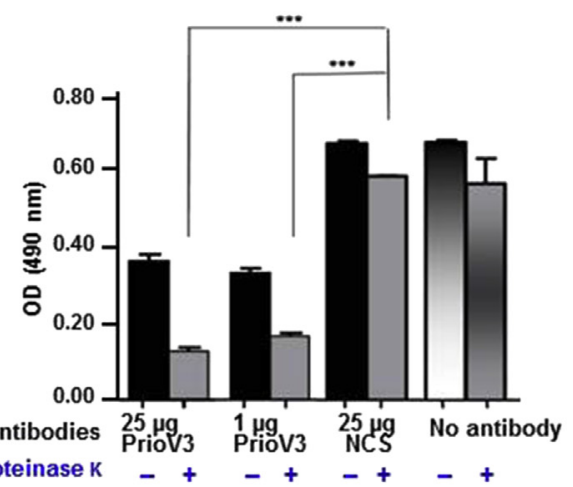

(E)

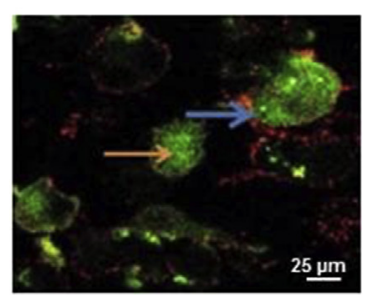

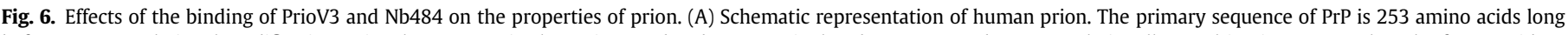

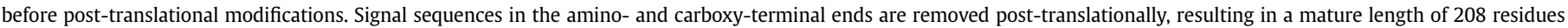

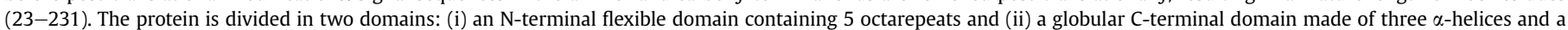

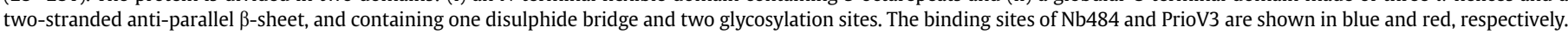

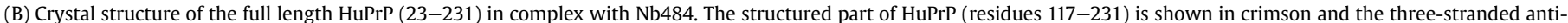

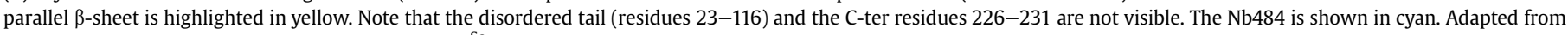

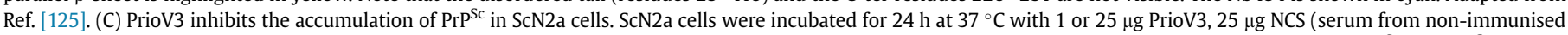

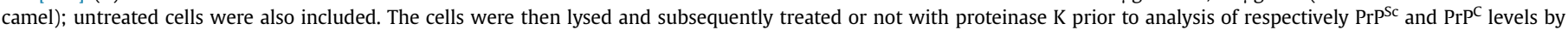

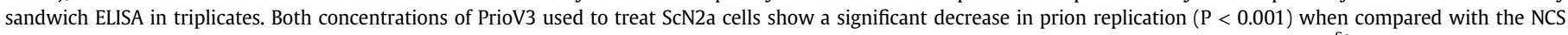

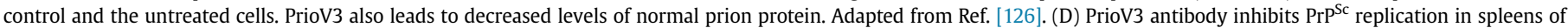

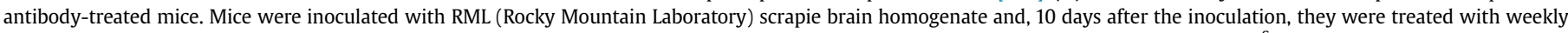

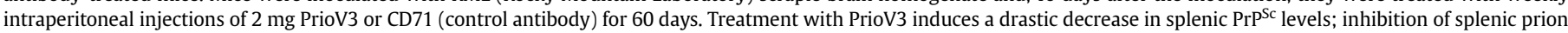

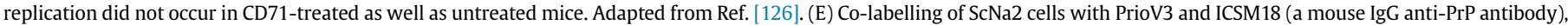

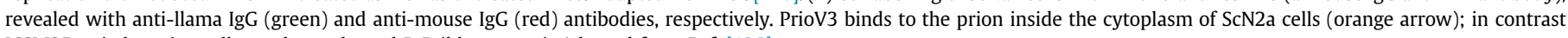
ICSM35 strictly stains cell membrane-bound PrP (blue arrow). Adapted from Ref. [126]. 
palindromic sequence (AGAAAAGA) encompassing residues 113-120 [121]. These conformational changes are believed to play a critical role for prion disease and transmissibility [124].

\subsubsection{PrP specific nanobodies Nb484 and PrioV3: generation and characterisation}

$\mathrm{Nb} 484$ has been selected by phage display from the blood of llamas immunised with recombinant murine $\operatorname{PrP}(\operatorname{MoPrP}(23-230)$ or murine PrP fragment MoPrP (89-230)). It was chosen among 14 nanobodies because it displayed the highest affinity for recombinant human PrP (HuPrP (23-231) and HuPrP (90-231)) [125]. Its epitope is discontinuous and includes residues $123-125$, the $\beta 2-\alpha 2$ loop (residues 164-170) and half of the $\alpha 2$-helix (residues 174-185) (Fig. 6A).

PrioV3, a specific nanobody to PrP, has been isolated by phage display from the blood of camels immunised with murine scrapie material adsorbed onto immunomagnetic beads [126]. Its epitope was identified via a pepscan approach by determining the ability of PrioV3 to bind to a series of 20-amino acid peptides spanning the region 99-230 of PrP. This analysis indicates that PrioV3 binds to a linear motif within the $\mathrm{C}$-terminal region of prion (i.e. residues 171-190), and of both $\mathrm{PrP}^{\mathrm{C}}$ and $\mathrm{PrP}^{\mathrm{Sc}}$ before and after digestion by proteinase $\mathrm{K}$ (Fig. 6A).

\subsubsection{Nb484 as structural probe}

$\mathrm{Nb} 484$ was used as a chaperone to crystallise for the first time the full length human $\operatorname{PrP}\left(\mathrm{HuPrP}^{\mathrm{C}}\right)$ and its C-terminal truncated version (residues 90-231) [125]. The X-ray diffracting crystal of both $\mathrm{PrP}$ forms in complex with $\mathrm{Nb} 484$ revealed unprecedented structural features at the $\mathrm{N}$-terminal of the palindromic sequence. Indeed, within the complex, about half of the palindromic conserved motif AGAAAAGA adopts a fully extended $\beta$-strand conformation (denoted $\beta 0$ ) that constitutes a three-stranded antiparallel $\beta$-sheet with the $\beta 1$ and $\beta 2$ strands (Fig. 6B). The 128-225 segment shares a structure that is very similar to the corresponding NMR structure of the free PrP although, upon the binding of Nb484, the $\beta 1$ extends from 3 to 7 residues and the $\beta 2-\alpha 2$ loop is significantly stabilised; the 23-116 residues remain highly unstructured. Therefore, this study demonstrated that the HuPrPC can adopt a more elaborate $\beta 0-\beta 1-\alpha 1-\beta 2-\alpha 2-\alpha 3$ structural organisation than the prion-like $\beta 1-\alpha 1-\beta 2-\alpha 2-\alpha 3$ anti-parallel $\beta$-ribbon. The propensity of the palindromic sequence to adopt a $\beta$-strand conformation suggests that it is probably involved in the $\beta$-enrichment of the $\operatorname{PrP}^{C}$ monomer, and these conformational changes could therefore constitute one of the early events of the conversion of $\operatorname{PrP}^{\mathrm{C}}$ to $\operatorname{Pr} \mathrm{P}^{\mathrm{Sc}}$.

\subsubsection{Nb484 and PrioV3 as mechanistic probes}

In vitro, $\mathrm{Nb} 484$ is able to slow down the aggregation into fibrils of murine $\operatorname{MoPrP}(23-230)$ in the presence of preformed murine $\mathrm{PrP}^{\mathrm{Sc}}$ seeds; the lag phase of aggregation kinetics is indeed extended by a factor $\sim 2(\sim 75 \mathrm{~h}$ versus $30 \mathrm{~h}$ in the presence and absence of Nb484, respectively) [125]. Moreover, Nb484 also inhibits the prion propagation in vivo: scrapie infected murine cells (ScGT1) treated with Nb484 show decreased $\mathrm{PrP}^{\mathrm{Sc}}$ levels compared to non-treated cells, the effect being Nb484 dose-dependent. In cells treated with $3.5 \mu \mathrm{M}$ Nb484, no PrP ${ }^{\mathrm{SC}}$ could be detected for at least 7 days and even 14 days after removal of the nanobody from the medium. It is not yet clearly determined if Nb484 inhibits the prion replication by steric hindrance or by preventing structural rearrangements that are essential for the formation of amyloidogenic intermediates [125]. The stabilisation of the $\beta 2-\alpha 2$ loop, which is part of the epitope and whose flexibility has been shown to be important for prion infection, may be particularly relevant.

PrioV3 alters the $\mathrm{PrP}^{\mathrm{C}}$ expression and induces a significant reduction in $\operatorname{PrP}^{\mathrm{Sc}}$ accumulation within the cells when added daily in the tissue culture medium (Fig. 6C). It is not clear, however, if these inhibitory effects are due to the recognition of $\operatorname{PrP}^{C}$ and/or $\mathrm{PrP}^{\mathrm{Sc}}$. Remarkably, the effects persist three days after the interruption of the addition of the nanobody [126]. Similar effects were observed with prion-infected GT1 cells (ScGT1) [127]. Moreover, no neurotoxic effects, measured by DNA fragmentation, were observed following treatment of the cells with the nanobody. Since PrioV3 targets both $\operatorname{PrP}^{C}$ and $\mathrm{PrP}^{\mathrm{Sc}}$, it could both block the conversion of $\mathrm{PrP}^{\mathrm{C}}$ into propagating infectious agents and, at the same time, it could neutralise the infectious template, $\operatorname{PrP}^{S c}$ [126]. Finally, a weekly injection of PrioV3 into mice, inoculated with scrapieinfected brain homogenate, leads to a marked inhibition of $\mathrm{PrP}^{\mathrm{Sc}}$ accumulation in the spleen of the animal (Fig. 6D) [126].

\subsubsection{PrioV3 is able to cross membranes}

PrioV 3 binds $\operatorname{PrP}^{\mathrm{C}}$ both at the cell surface and in the cytoplasm of ScN2a cells without cell permeabilisation, indicating that it is able to cross the cell membrane (Fig. 6E) [126]. The ability of the nanobody to cross the blood brain barrier (BBB) was established in vitro using two human BBB models. Moreover, following its intravenous injection, PrioV3 was widely distributed in the brain parenchyma of rats, demonstrating that it can also cross the BBB in vivo [126].

\subsubsection{Conclusions}

All together, these findings demonstrate the potential use of anti-prion nanobodies for the treatment of prion diseases and to better understand the molecular mechanism of aggregation. Compared to conventional antibodies such as the anti-prion murine mAb ICSM35, PrioV3 presents two main advantages: (i) first, it crosses the cytoplasmic membrane and the BBB, at least in rats, and can in consequence be active in cells and particularly in neurons, and (ii) secondly, it is devoid of toxicity at least in neuronal cell culture [126]. Moreover, Nb484 is an efficient crystallisation chaperone that has allowed getting for the first time high resolution structural feature (1.5 $\AA$ ) of a region of the protein encompassing part of the palindromic region: it forms new $\beta$-hairpin $(\beta 0-\beta 1)$ that can serve as a structural nucleus for the growth of intermolecular $\beta$-sheets.

\subsection{Polyadenylate binding protein nuclear 1 and oculopharyngeal muscular dystrophy}

The most common form of oculopharyngeal muscular dystrophy (OPMD) is an autosomal dominant disease that is caused by the expansion of GCG trinucleotide repeats in the coding sequence of the polyadenylate-binding protein nuclear 1 (PABPN1), translated into a poly(Ala) stretch [128]. The wild-type protein bears 10 alanines at its N-terminus; mutant proteins (mPABPN1) in which the polyA tract is extended to $12-17$ residues cause the disease and the longer the tract the more severe the disease [128]. The characteristic feature of OPMD is the presence of filamentous intranuclear inclusions made of mPABPN1 and resistant to $\mathrm{KCl}$ treatment [128]. Although the exact pathological mechanism underlying OPMD is still poorly understood, a body of evidence suggests that it is associated, at least in part, with the formation of aggregates and especially of oligomers or micro-aggregates made by the mutant proteins $[129,130]$. Although probably all the skeletal muscles are affected, the histologic changes are however most pronounced in a limited set of skeletal muscles (i.e. extraocular, lingual, pharyngeal and diaphragmatic muscles) [128]. OPMD usually starts in the fifth or sixth decade of life with the main symptoms being eyelid drooping and difficulty in swallowing due to a weakness in the levator palpebrae superioris and pharyngeal muscles, respectively [129]. The disease does not shorten the life expectancy of the 
affected patients; it however significantly impairs the quality of their life due to frequent aspiration pneumonia and difficulties in swallowing which lead to malnutrition [128]. The largest OPMD cluster is in French-Canadian population with an estimated prevalence of 1:1,000; in Europe, the estimated prevalence is $1: 100,000$ [128].

PABPN1 is a nuclear ubiquitously expressed multifunctional protein which is involved in pre-mRNA polyadenylation, transcription regulation, and mRNA nucleocytoplasmic transport in presumably all tissues [129]. It is a 306 residue multi-domain protein (MM $\sim 32.8 \mathrm{kDa}$ ) (Fig. $7 \mathrm{~A}$ ) that can be divided in three domains [128,129]. (i) An acidic N-terminus domain (residues 1-161) starts with a stretch of 10 alanines. Moreover, part of this domain (residues 119-146), which is essential for the interaction of PABPN1 with the poly-A polymerase, is predicted to form a coiled-coil structure (CC). (ii) A RNA recognition motif (RRM, residues 162-257) which consists of four parallel $\beta$-sheets coupled with two perpendicular $\alpha$-helices. And (iii) a basic C-terminal domain rich in dimethylated arginine (residues 258-306); this domain contains a nuclear localisation signal (NLS). The protein also contains two oligomerisation domains: one overlapping the RNA binding domain (residues 155-294) and the other comprising the NLS sequence (residues 264-306).

Both the wild-type and mPABPN1 are aggregation-prone but only the mutant form is pathogenic. When bound to the poly- $A$ mRNA, PABPN1 forms in vitro, via its oligomerisation domains,
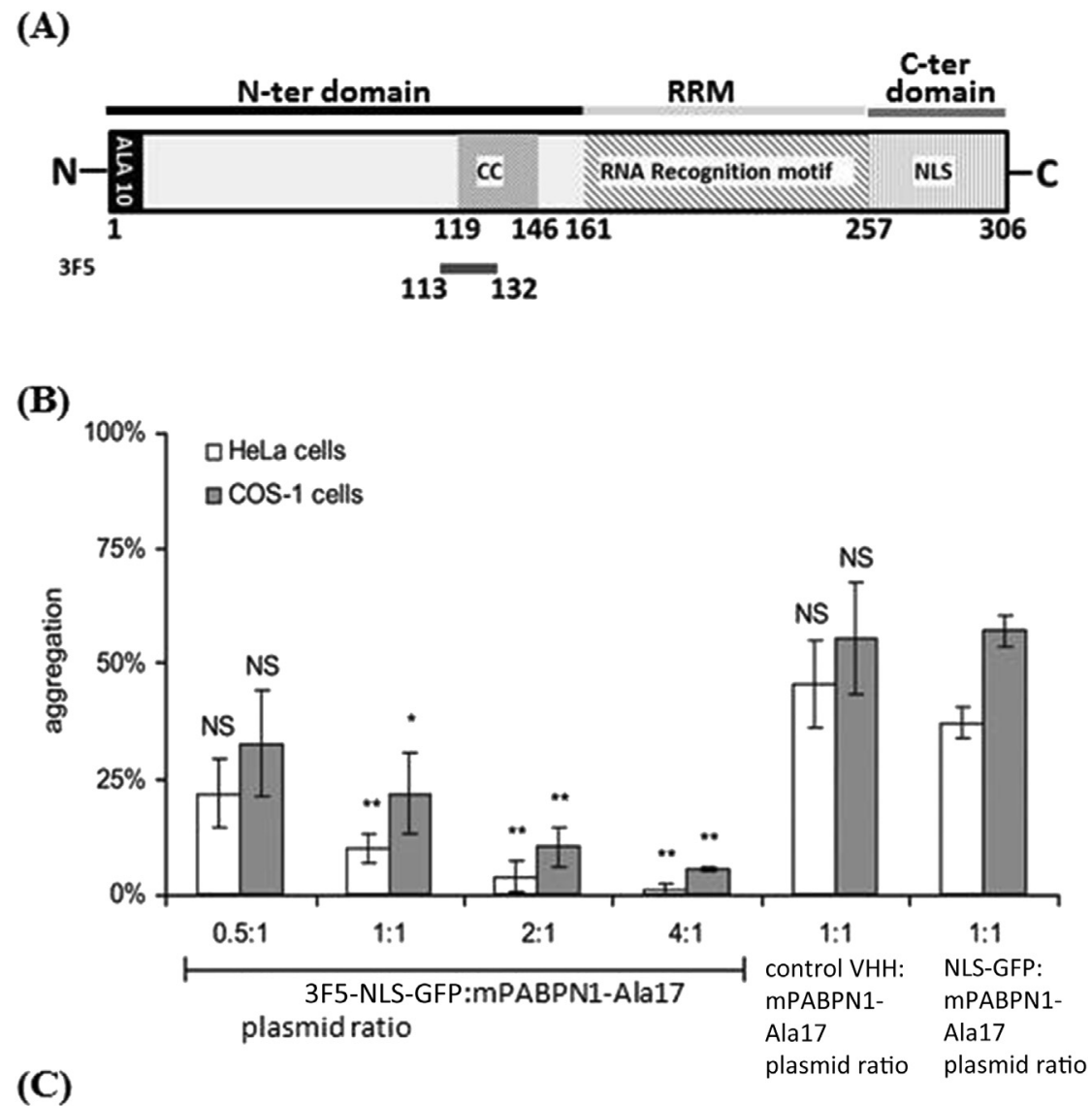

\begin{tabular}{|c|c|c|c|c|}
\hline \multirow{2}{*}{ Nanobody } & \multicolumn{2}{|c|}{$\%$ of flies with a bnormal wing posture } & \multirow{2}{*}{$\begin{array}{c}\text { Suppressor } \\
\text { activity }\end{array}$} & \multirow{2}{*}{$\begin{array}{c}\text { Number } \\
\text { of lines }\end{array}$} \\
\hline & Day 6 & Day 11 & & \\
\hline- & $87 \%$ & $87 \%$ & - & - \\
\hline $3 F 5$ & 3 to $32 \%$ & 3 to $32 \%$ & +++ & 4 \\
\hline $3 E 9$ & 31 to $41 \%$ & 31 to $41 \%$ & ++ & 2 \\
\hline $3 A 9$ & 44 to $62 \%$ & 44 to $62 \%$ & + & 5 \\
\hline$\# 08$ & 64 to $76 \%$ & 64 to $76 \%$ & \pm & 3 \\
\hline$\# 18$ & 61 to $78 \%$ & 61 to $78 \%$ & \pm & 4 \\
\hline$\# 29$ & 60 to $68 \%$ & 60 to $68 \%$ & \pm & 3 \\
\hline
\end{tabular}

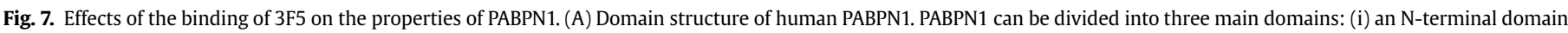

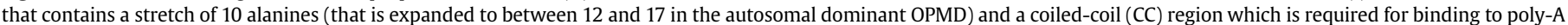

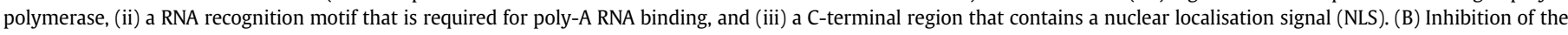

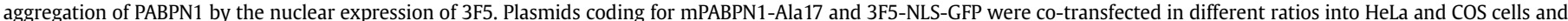

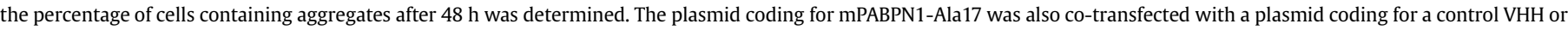

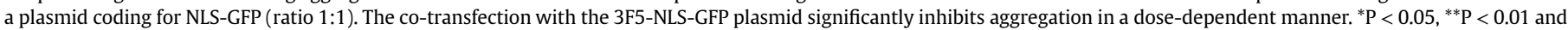

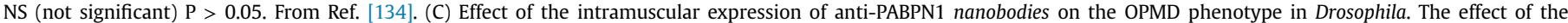

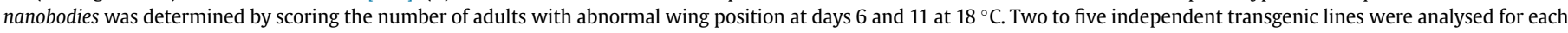

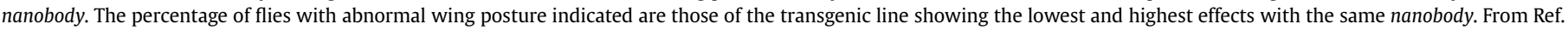
[136]. 
both linear filaments and discrete-size, compact oligomeric particles which do not resist to $\mathrm{KCl}$ treatment contrary to the aggregates formed by mPABPN1 [131]. Deletion of one of the oligomerisation domains prevents the pathological aggregation of the mutant form of PABPN1 containing 17 alanines (mPABPN1Ala17) into KCl-resistant aggregates [130,131]. This observation indicates that, like for the aggregation of proteins containing a polyglutamine tract, the regions outside the alanine repeat have their own role to play [132] and suggests a multi-domain aggregation mechanism. Differences in the aggregation kinetics and/or pathway by the wild-type and mPABPN1 could explain their differences in toxicity [133].

\subsubsection{Generation and characterisation of PABPN1 specific nanobodies}

A series of PABPN1 specific nanobodies have been selected from a large $\left(5 \cdot 10^{9}\right.$ individual clones) non-immune llama HCAb library using both recombinant human and bovine PABPN1 as panning antigens [134]. A total of six different nanobodies with affinities for PABPN1 ranging from 5 to $57 \mathrm{nM}$ were selected. The epitope of the nanobody exhibiting the highest affinity (i.e. $5 \mathrm{nM}$ ), referred to as 3F5, was mapped using a panel of truncated and point-mutation recombinant PABPN1 proteins. It is located between residues 113 and 132 which largely overlap with the region of the protein that is predicted to form a coiled-coil domain (Fig. 7A), and residues 126, 129 and 131 are essential for the binding of the nanobody. This conclusion was also supported by an NMR study [135]. Two other nanobodies (3A9 and 3E9) recognise an epitope in the 1-155 region, and the other three (\#08, \#18 and \#29) were selected by epitope-masking using 3F5 [136] and thus are likely to recognise an epitope different from that of 3F5. 3F5 detects PABPN1 in the nuclear fraction of HeLa cells [134]. Moreover, 3F5 labels the nucleus of cultured cells (HeLa and COS-1) and human muscles on cryosections [134].

\subsubsection{F5 expressed in cells affects in vivo aggregation}

The effect of intracellular expression of 3F5 was investigated in cellular PABPN1 aggregation models. A plasmid coding for 3F5 in fusion with a nuclear localisation signal (NLS) and the green fluorescent protein (GFP), referred to as 3F5-NLS-GFP, was transfected into COS-1 and HeLa cells. The GFP signal was observed only in the nucleus, indicating both a successful expression of the nanobody and its targeting into the nucleus [134]. In cells co-transfected with a constant amount of plasmid coding for murine mPABPN1-Ala17 and an increasing amount of plasmid coding for 3F5-NLS-GFP, the nanobody drastically prevents the aggregation of mPABPN1-Ala17 in a dose-dependent manner (Fig. 7B). Moreover, the expression of the nanobody is not toxic for the cell (i.e. it does not modify the cell viability) and does not affect the expression of mPABPN1-Ala17. The decreased aggregation in the presence of 3F5 is therefore a direct effect of the nanobody binding and is not due to a reduced amount of the expressed mutant protein. The effects of 3F5 are due to very specific interactions since the binding of the other PABPN1-specific nanobodies recognising different epitopes does not inhibit the aggregation [134]. Moreover, transfection with the plasmid coding for $3 F 5$ in HeLa and COS cells already containing mPABPN1-Ala17 intranuclear aggregates, results in a significant decrease in the amount of the latter [134]). In another study, using U2OS cells, 3F5 was however found to reverse the formation oligomers but not that of insoluble aggregates [133].

\subsubsection{F5 expressed in an animal model}

The effects of the six nanobodies were investigated in vivo using a Drosophila model that recapitulates the characteristics of the disease, in particular (i) muscle degeneration, the myofibril sarcomeric structure being strongly disorganised with broken Z-bands and the absence of M-band, (ii) gene deregulation and (iii) formation of dense PABPN1 nuclear inclusions in muscle cells composed of fibrillar aggregates. These modifications cause an abnormal wing posture [136]. The capacity of the six PABPN1 nanobodies to decrease OPMD phenotypes in vivo was assayed by co-expressing PABPN1-Ala17 with each of them in muscles using the Mch-Gal4 driver system. The capacity to decrease OPMD phenotype is different for each nanobody (Fig. 7C). The nanobodies \#08, \#18 and \#29 have a very weak effect on suppressing abnormal wing position. 3E9 and 3A9 have an intermediate effect, whereas 3F5 is very effective in suppressing the abnormal wing posture (Fig. 7C), and its effect is dose-dependent [136]. The lower efficiency of the nanobodies to decrease the phenotype was not correlated to a lower expression level; it is likely that it is instead correlated with the epitope they recognise and/or with their affinity for PABPN1. Moreover, 3F5 does not interfere with the aggregation process in flies expressing mutant PABPN1 in which the coiled-coil region has been deleted, and does not rescue the disease phenotype of these flies [136]. Thus, the curative effects of 3F5 depend on its ability to bind to the coiled-coil region of PABPN1 in vivo.

The co-expression of 3F5 with mPABPN1-Ala17 prevents muscle degeneration in Drosophilia and restores a normal appearance of the muscle fibres [136]. It does not reduce the number of nuclei containing aggregates; the size of the aggregates is however reduced by a factor up to 2.5 and the aggregates formed are more dispersed. These observations suggest that 3F5 could interfere with intermediate species such as oligomers or micro-aggregates and prevent their toxicity [136]. A transcriptome analysis showed that the co-expression of 3F5 decreases the OPMD-induced gene deregulation. This effect was the strongest in adults at day 2 but persists during the whole lifespan of the animals [136]. All together these results strongly suggest that the expression of the nanobody prevents muscle degeneration in vivo by altering the nuclear aggregation of mPABPN1-Ala17 and restoring gene expression. The epitope of 3F5 is located in the coiled-coil region of the protein and thus is also present on the wild-type protein. The expression of 3F5 in human cells could therefore potentially affect normal functions of PABPN1 although it does not induce muscle degeneration in Drosophila and does not reduce the viability of cell models.

\subsubsection{Conclusions}

These studies show that nanobodies can efficiently be expressed intracellularly, in cell cultures and animal models, in a functional form using conventional expression vectors. Again, the easy engineering of the nanobodies are important (i) to direct the expression of the nanobody in specific cellular compartments (i.e. by fusing it to an addressing signal) and (ii) to fuse it to an imaging agent (i.e. the GFP).

\subsection{A $\beta$ peptide and Alzheimer's disease}

Alzheimer's disease (AD), the most common neurodegenerative disorder, is characterised by two features: (i) the intraneuronal accumulation of hyperphosphorylated tau protein in the form of neurofibrillary tangles, and (ii) the formation of extracellular amyloid plaques, composed of the $A \beta$ peptide (also referred to as senile plaques) in the central nervous system $[137,138]$. The accumulation of these aggregates, or some of their precursor oligomeric species, is thought to cause the loss of neurons, notably in the hippocampus, leading to memory deficiency and cognitive dysfunctions [139]. A $\beta$ peptides arise from a proteolytic cleavage of the transmembrane glycoprotein amyloid precursor protein (APP) following the action of two secretases [140]. First, the $\beta$-secretase generates the APPs $\beta$ fragment (secreted in the plasma) and the C99 fragment (still membrane anchored) [141]. This latter is then cleaved by the $\gamma$ secretase into a variety of peptides including the amyloidogenic 
$A \beta_{40}$ and $A \beta_{42}$ fragments. APP could also be first proteolysed by the $\alpha$-secretase and this leads to the production of non-amyloidogenic peptides.

$A \beta$ peptides are produced in the brain and the cerebrospinal fluid (CSF) of healthy humans; $A \beta_{40}$ is the most abundant secreted species, while $A \beta_{42}$ has a stronger tendency to aggregate and is believed to be the first species to deposit in senile plaques [142-144]. No precise physiological role is yet known for $A \beta$ peptides but a study has shown that $A \beta$ modulates synaptic strength while its production and secretion are regulated by neuronal activity. Thus, $A \beta$ could have a negative feedback function and may play a role in normal synaptic physiology [145]. In affected patients, the over-production of $A \beta$ peptides or an increased proportion of $A \beta_{42}$ relating to $A \beta_{40}$ appear sufficient to cause the disease [146]. It was proposed that the $A \beta_{42}: A \beta_{40}$ ratio, rather than the absolute amounts of the two peptides, is critical for the induction of neurotoxic conformations [147]. In addition to sporadic $A D$, an early-onset familial form of the disease has also been described and is associated with genetic mutations. More than 230 mutations in $\gamma$-secretases (i.e., presenilins PS1 and PS2) and in APP genes, including the A $\beta$-peptide sequence (Fig. 8A), have been identified, leading to an excessive production of $A \beta$ peptides and/or to an increased propensity of the mutant peptides to aggregate $[148,149]$. Nowadays, within the United States, AD is the 6th leading cause of death [150].

$A \beta$ peptides are assumed to have an $\alpha$-helical conformation as part of APP in the membrane. By contrast, when released in solution, they seem to predominantly adopt unstructured conformations that only transiently form helical secondary structure elements [151]. On the in vitro pathway of fibril formation by $A \beta_{42}$, several intermediate species are formed, including SDS-stable oligomers, $A \beta$-derived diffusible ligands (ADDLs) and protofibrils.

It is now widely accepted that these soluble pre-fibrillar assemblies are more cytotoxic to neurons while fibrillar aggregates may not play the main role in pathogenesis [146,152]. The A $\beta$ peptide fibrils consist in a layered structure in which three cross- $\beta$ subunits are arranged in six tightly stacked $\beta$-sheet layers with an anti-parallel hydrophobic-hydrophobic (residues 30-40) and an anti-parallel polar-polar interface (residues 10-22) [153].

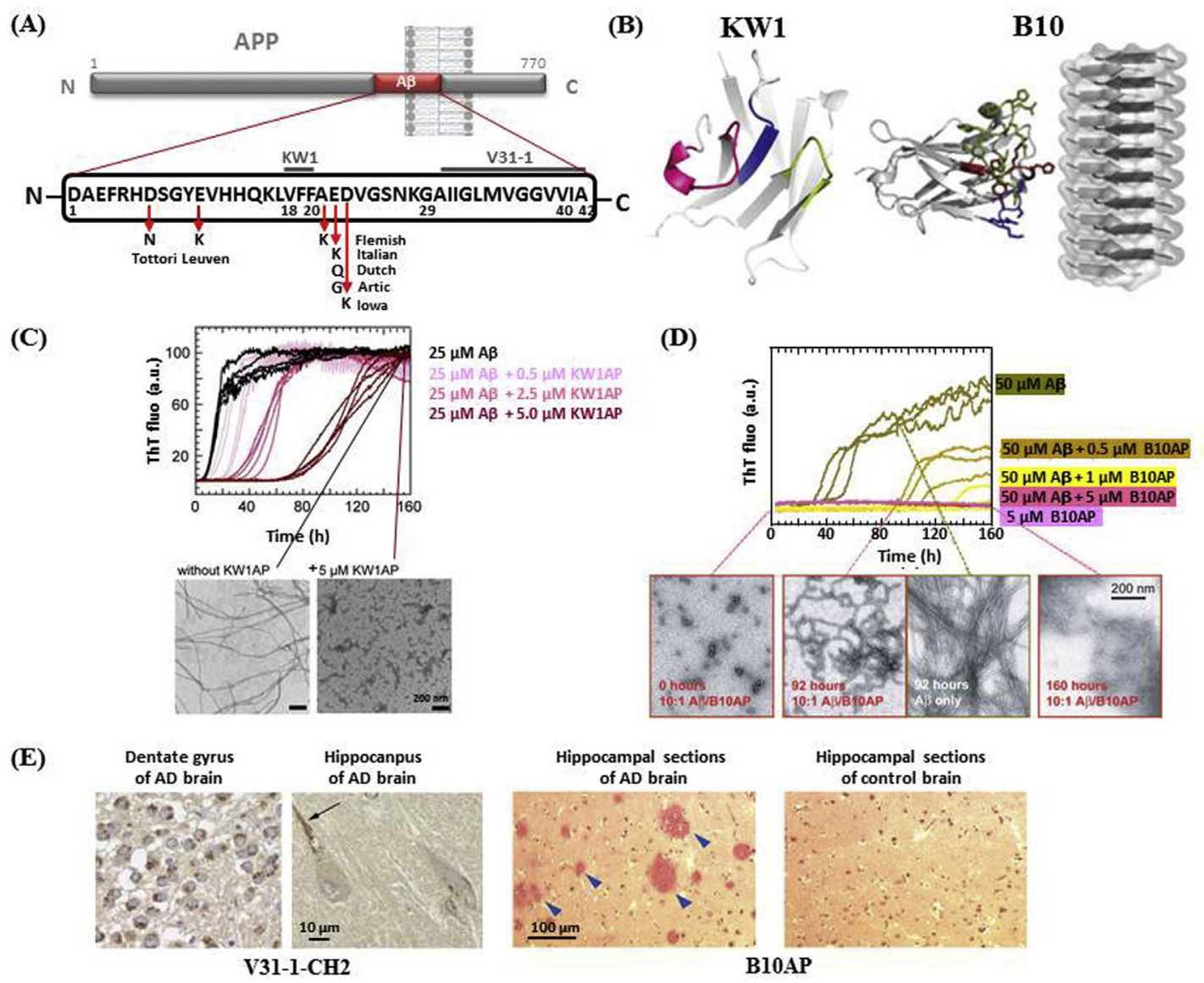

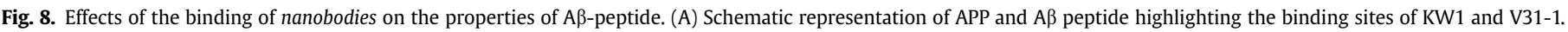

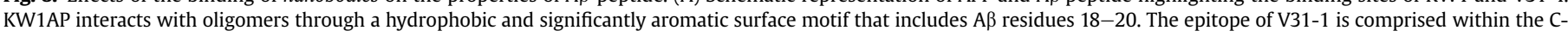

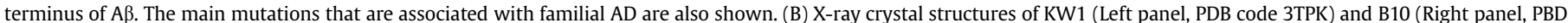

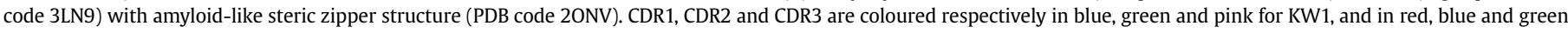

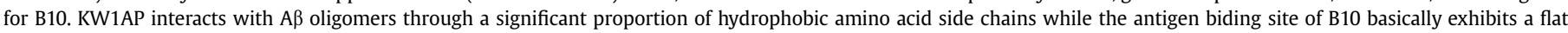

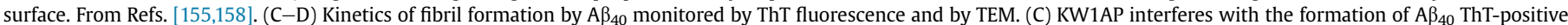

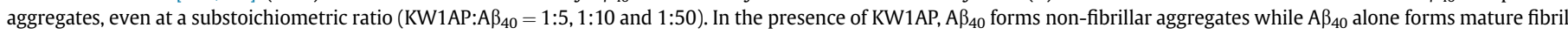

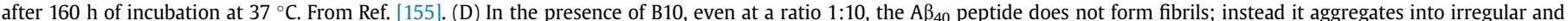

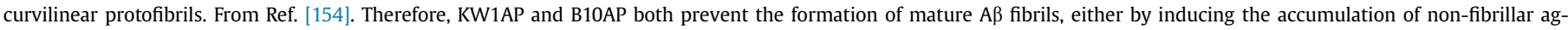

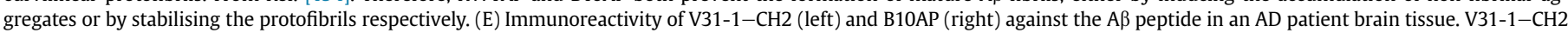

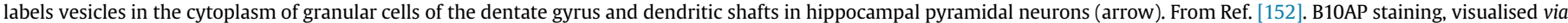

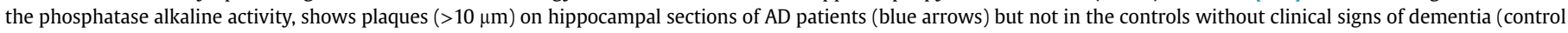
brain). From Ref. [154]. 


\subsubsection{Generation of $A \beta$ specific nanobodies}

A series of nanobodies, specific to particular $A \beta$ peptide species (i.e. monomers, oligomers and/or fibrils), have been generated and characterised in detail; they are referred to as B10 [154], KW1 [155], V31-1 [152], PrioAD12 and PrioAD13 [127] and ni3A [156].

3.6.1.1. B10 and KW1. B10 and KW1 have been retrieved from fully synthetic libraries coding for nanobodies, using respectively partially biotinylated fibrils and oligomers of $A \beta_{40}$ as targets, immobilised on the surface of streptavidin-coated magnetic beads $[154,155]$. The selection was carried out in the presence of a 10-fold molar excess of freshly disaggregated $A \beta_{40}$ peptide (i.e. largely monomeric) in order to avoid the selection nanobodies that bind to the monomeric form of $A \beta_{40}$. B10 and KW1 were fused to Escherichia coli alkaline phosphatase (AP), a homodimeric protein, and the bivalent chimeric proteins are called respectively B10AP and KW1AP. The advantage of using these chimeras is threefold: (i) they have a higher apparent affinity for their respective epitope than B10 and KW1, (ii) they increase the solubility of the nanobodies, and (iii) the AP moiety serves as reporter (based on an enzymatic colour assay) for the detection of nanobodies in immuno-blot and immunochemistry without the need to use a secondary antibody, and for transmission electron microscopy (based on the catalysis of the formation of cerium phosphate, an electron-dense precipitate that accumulates in the vicinity of the active site). Here, again, the easiness with which nanobodies manifold constructs can be engineered constitutes an important advantage.

3.6.1.2. V31-1. V31-1 was selected, using $A \beta_{42}$ as bait, from a library made from the blood of a llama immunised with $A \beta_{42}$ incubated overnight at $37{ }^{\circ} \mathrm{C}$, corresponding to a mixture of monomers, oligomers and fibrils [152]. As B10 and KW1, this nanobody was engineered as a multifold construct for imaging purpose; it was fused to the $\mathrm{CH} 2$ domain of an alpaca HCAb (V31-CH2) for immunostaining studies (Fig. 8E). The signal could therefore be amplified using rabbit anti-alpaca Ig polyclonal antibodies as secondary antibodies which are labelled by an anti-rabbit biotinylated goat polyclonal antibody. The streptavidin-peroxidase complexes could then be revealed with diaminobenzidine [152].

3.6.1.3. PrioAD12 and PrioAD13. PrioAD12 and PrioAD13 were isolated from a library made from the blood of three alpagas immunised with homogenised brain of an $A D$ patient absorbed immunomagnetic beads [127]. PrioAD12 is specific of $A \beta_{40}$ while PrioAD13 is specific of $A \beta_{42}$.

3.6.1.4. ni3A. ni3A was isolated from a non-immune llama-derived phagemid library and selected by phage display against $A \beta_{42}$ [157]. Two successive rounds of selection were performed, using coated synthetic $A \beta_{42}$ as target. ni3A recognises $A \beta_{42}$ with a high affinity $\left(K_{D}=39 \mathrm{nM}\right)$ while it does not bind to $A \beta_{40}$.

\subsubsection{A $\beta$ nanobodies as structural probes}

3.6.2.1. V31-1 specifically binds to monomers and small oligomers (from dimers to 12-mers) of $A \beta$. V31-1 has a high affinity for $A \beta_{42}$ $\left(K_{D} \sim 30 \mathrm{nM}\right)$ and does not recognise the $A \beta_{40}$ peptide nor the $1-11$, $10-20,15-25$ and $22-35$ peptides from $A \beta$ [152]. However, it binds to the $29-40$ and 33-42 fragments, suggesting that it recognises an epitope located at the $\mathrm{C}$-terminal end of $\mathrm{A} \beta$ peptide. Moreover, V311 was found to be able to bind to monomers, dimers, trimers and tetramers as well as 12-mers but not higher molecular mass, SDSinsoluble, oligomers nor fibrils, suggesting that in these species the C-terminus is not accessible. The high specificity of this nanobody for low-MM oligomers made of $A \beta_{42}$ suggests that it could have a great potential for diagnosis and detection of $A \beta$ oligomers in patients' brains.

3.6.2.2. KW1 specifically binds to $A \beta_{40}$ oligomers. KW1 is specific to $\mathrm{A} \beta_{40}$ oligomers: it does not bind to fibrils nor to disaggregated $A \beta_{40}$ peptides [155]. It interacts preferentially with non-fibrillar and often heterogenous high-MM $A \beta_{40}$ oligomers which have an isotropic shape and diameters of $8-30 \mathrm{~nm}$. Remarkably, KW1AP does not bind to $A \beta_{42}$ oligomers although these cannot be differentiated from $A \beta_{40}$ oligomers by Fourier transform infrared spectroscopy (FTIR) and TEM. KW1 can therefore probe very subtle and surface-inherent properties of oligomers. The X-ray structure of KW1 shows that it possesses a large hydrophobic cavity that is exposed to the solvent (Fig. 8B). The binding properties of KW1AP with a series of $A \beta_{40}$ mutant peptides, in combination with peptide spot arrays and NMR studies, suggest that it interacts with oligomers through a hydrophobic and significantly aromatic surface motif that includes $A \beta$ residues $18-20$ (Fig. 8A).

3.6.2.3. B10 is specific to protofibrils and fibrils. B10 specifically binds to protofibrils and mature amyloid fibrils formed by the $A \beta_{40}$ peptide. Its affinity (i.e. $K_{D}$ ) for $A \beta_{40}$ amyloid fibrils, determined by $\mathrm{SPR}$, is $\sim 475 \mathrm{nM}$, and the stoichiometry of binding is $1: 10$ (B10:A $\left.\beta_{40}\right)$. The affinity of B10AP, which is bivalent, is 50 -fold higher (i.e. $K_{D}$ $\sim 7.2 \mathrm{nM}$ ) [154]. B10 does not recognise other conformers of the $A \beta$ peptide such as disaggregated peptides or non-fibrillar $A \beta$ oligomeric species exhibiting a very high $\beta$-sheet content similar to that of amyloid fibrils [154]. The presence of a $\beta$-sheet structure is therefore not sufficient to explain the B10 binding. Interestingly, B10 is able to bind to different types of amyloid fibrils formed in vitro by $A \beta_{40}$ and $A \beta_{42}$ under different conditions of incubation. B10 also binds to: (i) fibrils formed in vitro from a broad range of amyloid proteins (i.e. insulin, glucagon, $\beta 2 \mathrm{~m}$ ), and to amyloid fibrils from $\mathrm{D}$-amino and $\mathrm{L}-$ amino acid peptides; (ii) tissue-extracted amyloid fibrils composed of serum amyloid A (SAA) protein and Ig light chain (AL) [24]; (iii) hippocampal sections from Alzheimer's patients (Fig. 8E)[154]. Importantly, these observations indicate that fibrils formed in vitro and in vivo, at least by the $A \beta$ peptide, do share some basic structural features. The B10-epitope density at the surface of the fibrils as well as B10AP staining intensity both vary, however, for the different fibrils. More importantly, B10 does not recognise - at all - some amyloid fibrils formed in vitro (i.e. fibrils made of $A \beta_{16-22}$, apomyoglobin and PABPN1), nor some amyloid tissues deposits [24]. The recognition of amyloid fibrils by B10 cannot, therefore, solely be explained by an amyloid-specific polypeptide fold since, despite their common backbone conformation, not all amyloid fibrils are recognised by B10 [24].

The easiness with which mutations can be introduced in the gene of nanobodies and with which nanobodies can be produced in large quantities allowed a large number of mutants (i.e. 23 variants corresponding to single-site mutagenesis of the 23 residues within CDRs $1-3$ ) to be produced and characterised, in order to understand the molecular basis of the specificity of B10 [158]. The results of this systematic mutagenesis approach show that the basic residues (positively-charged residues) belonging to CDRs create a strongly positive electrostatic potential at the B10 binding surface, leading to electrostatic interactions with anionic groups present on some fibril surfaces. Moreover, the binding of B10 to amyloid fibrils composed of $A \beta_{40}$, insulin and G-helix peptide from sperm whale myoglobin is significantly reduced when the carboxyl groups of the fibrils are chemically modified [158]. The conformational specificity of B10 for amyloid fibrils seems therefore to depend upon specific electrostatic interactions with highly regular and anionic surface pattern common, at least locally, to different but not all amyloid fibrils, and not on the recognition of an amyloid generic backbone 
conformation. Finally, the X-ray structure of B10 shows that its three CDRs constitute a positively charged flat surface (Fig. 8B) [158]. The B10 molecular recognition reaction can therefore be described as an association between two relatively flat but complementary surfaces exhibiting opposite charges [158].

\subsubsection{A $\beta$ nanobodies as mechanistic probes}

3.6.3.1. B10 blocks amyloid fibril formation at the protofibril stage. The binding of B10AP to preformed $A \beta$ fibrils does not lead to their significant disaggregation. Moreover, in the presence of B10 (even at a ratio $1: 10$ ), the $A \beta_{40}$ peptide does not form amyloid fibrils instead, it aggregates into irregular and curvilinear protofibrils (Fig. 8D). The latter species display an amyloid-like cross- $\beta$ core structure with a higher susceptibility toward proteolytic digestion than mature fibrils, and they interact only weakly with ThT or Congo red (CR). B10 therefore prevents the formation of mature amyloid fibrils by arresting fibril formation at the protofibril stage. The fact that B10AP binds both protofibrils and mature amyloid fibrils suggests that these two species possess the $B 10$ epitope at their surface. Kinetics of $A \beta_{40}$ fibril formation monitored both by ThT fluorescence and dot-blot experiments with B10AP indicate that the formation of this epitope preceeds the formation of ThT-positive fibrils. B10 is therefore likely to inhibit the formation of mature fibrils by preventing the selfassociation of protofibrils [154].

3.6.3.2. KW1AP prevents the formation of mature amyloid fibrils and leads to the formation of non-fibrillar aggregates. KW1, which is specific to high-MM $A \beta_{40}$ oligomers, interferes with the formation of $A \beta_{40}$ ThT-positive aggregates, even at a substoichiometric ratio (KW1AP:A $\beta_{40}=1: 5,1: 10$ and 1:50) (Fig. 8C). The presence of both KW1AP (Fig. 8C) and KW1 significantly increases the lag phase of the $A \beta_{40}$ aggregation kinetics, suggesting that (i) the presence of the alkaline phosphatase moiety does not perturbs the effects of the nanobody and (ii) the effects of KW1 is similar weather it is monomeric or dimeric (in KW1AP). Although the aggregates formed in the presence of KW1AP or KW1 bind ThT, they appear as non-fibrillar by TEM analysis [155,159]. Moreover, they exhibit a higher surface hydrophobicity than the fibrils formed in the absence of KW1, as revealed by an increased anilino naphthalenesulfonic acid (ANS) binding. Finally, as B10, KW1AP does not significantly disaggregate preformed $A \beta$ amyloid fibrils [155].

3.6.3.3. V31-1 inhibits amyloid fibril formation. When present at an equimolar ratio, V31-1, which specifically binds to monomers, dimers, trimers, tetramers and 12-mers of $A \beta_{42}$, inhibits the formation of $A \beta_{42}$ amyloid fibrils. Dynamic light scattering (DLS) experiments indicate that, in the presence of an equimolar concentration of V31-1, A $\beta_{42}$ forms low-MM aggregates $\left(R_{H}\right.$ of $10-25 \mathrm{~nm}$ and $150-200 \mathrm{~nm})$. However, high-MM species $\left(\mathrm{R}_{\mathrm{H}}\right.$ of $600-700 \mathrm{~nm}$ ) formed in the absence of V31-1 are not observed. This suggests that this nanobody prevents the formation of highMM aggregates [152].

It should be noted that several conventional antibodies are raised against $A \beta$ oligomers; most of them however also bind to fibrils [160-162] and are therefore less specific than V31-1. Moreover, other antibodies that are specific to the C-terminus of $A \beta_{42}$ do not inhibit the aggregation [163], contrary to V31-1. Altogether, these observations suggest that V31-1 binds to an epitope that is not recognised by conventional antibodies because it is less immunogenic or less accessible. It has been suggested that the $C$ terminus of $A \beta_{42}$ is more rigid than that of $A \beta_{40}$, may be reflecting a pre-ordered structure for the acquisition of the $\beta$-conformation present in soluble oligomers and fibrils [164]. The $A \beta_{42}$ C-terminus may therefore act as an internal seed for aggregation and V31-1, that could recognise such a structure in oligomers, is thus able to inhibit the formation of fibrils [152].

\subsubsection{Nanobodies to detect $A \beta$ in cells and tissue biopsies}

The distribution of V31-1-specific immunoreactivity in brain of patients with AD shows that this nanobody binds mostly intraneuronal $A \beta$ peptides and stains only faintly extracellular amyloid plaques or neurofibrillary tangles [152]. Western-blot immunoassays, performed on formic acid fractions obtained from AD brains, further indicate that V31-1 detects $A \beta$ oligomers in brain tissues. KW1AP binds to oligomeric species derived from occipital and temporal brain regions of an AD patient. B10 detects amyloid fibrils in tissue biopsies, although it stains only a small fraction (24\%) of the large series (106) of amyloid samples originating from a large variety of organs and types of amyloidosis [165]. PrioAD12 efficiently binds to $A \beta$ plaques on brain sections from a patient with $\mathrm{AD}$; but no staining is observed with control brain [127]. Moreover, staining of AD brain sections with FTIC-labelled PrioAD12 and texas red-labelled lysosomal-associated membrane protein 1 co-localise suggesting that the species recognised by PrioAD12 are located in the late endosomal/lysosomal compartment [127]. All together, these results indicate that some of the nanobodies could be very useful as diagnostic tools.

\subsubsection{Effects of nanobodies on the $A \beta$ toxicity}

V31-1 prevents the $A \beta$-oligomers induced toxicity towards SK$\mathrm{N}-\mathrm{SH}$ cells, probably by preventing their interaction with the cells [152].

The co-expression of $B 10$ with a series of $A \beta$-peptides $\left(A \beta_{40}\right.$, $A \beta_{42}, A \beta_{42 A r c t i c}$ (Fig. 8A)) in Drosophila melanogaster does not result in any phenotypic changes compared to the expression of the $A \beta$ peptides alone [159] despite the facts that B10 (i) is able to bind fibrils formed in vitro by the three peptides and (ii) promotes, in vitro, the formation of protofibrils instead of fibrils.

When added to a suspension of in vitro preformed $A \beta_{40}$ oligomers, KW1AP antagonises the toxicity of the latter: they are not able anymore to disrupt synaptic plasticity and perturbs long term potentiation (LTP) in cultured murine brain slices [155]. The antibody is thought to act by preventing the binding of oligomers to cellular surfaces or receptors. However, oligomers formed in vitro in the presence of KW1 are highly toxic: they reduce the synaptic potentiation in murine brain slices and the metabolic activity of human neuroblastoma [159]. Similarly, when co-expressed in flies with $A \beta_{40}$, KW1 promotes toxicity: the median survival time of flies expressing both KW1 and $A \beta_{40}$ is $28 \pm 1.1$ days while that of flies expressing only $A \beta_{40}$ or KW1 is respectively $43 \pm 0.6$ and $41 \pm 0.9$ days [159]. The toxicity observed with oligomers formed in the presence of KW1 is likely due to the fact that these oligomers expose a more hydrophobic surface that favour their interaction with cellular surfaces or receptors (see above).

In summary, these studies show that KW1 can prevent or enhance $A \beta$ toxicity depending on the context and especially on the time point in the aggregation process at which it is added. It may block the binding of preformed oligomers to their cellular receptors thus inhibiting their toxicity; but it can also modulate the peptide self-assembly reaction and leads to the formation of more toxic species. The biological consequence of a nanobody, and probably of any ligand, is therefore difficult to predict and this highlights the complexity in developing therapeutic strategies to treat amyloidoses.

\subsection{6. ni3A crosses the $B B B$}

ni3A significantly transmigrates across a model BBB in vitro at $37{ }^{\circ} \mathrm{C}$ [157]. On the other hand, ni8B, a nanobody that differs from ni3A by only three amino acids in the $\mathrm{N}$-terminus at positions 13,14 , 
and 16, transmigrates much less the BBB suggesting that these three amino acids, which are unusual for $\mathrm{V}_{\mathrm{H}} \mathrm{Hs}$, play a critical role. To elucidate the role of these amino acids, a chimeric nanobody was created; it consists in the sequence of va2E, a nanobody that cannot significantly cross the model BBB, in which the FR1 containing the three unusual amino acid has been replaced by that of ni3A [156]. The chimeric protein has a significantly higher transmigration rate compared to the parental va2E, indicating that the three amino acids that originate from ni3A facilitate the efficient BBB crossing of the nanobodies. The rate of model BBB crossing of the chimeric $\mathrm{V}_{\mathrm{H}} \mathrm{H}$ was however much lower than that of ni3A, indicating the other features of ni3A were responsible of this property. The high percentage of charged amino acids of ni3A could explain, at least in part, why ni3A outperforms other nanobodies in crossing the model BBB. Moreover, there was almost no transmigration of ni3A across the barrier at $4{ }^{\circ} \mathrm{C}$, suggesting that it crosses the BBB by an active transport.

\section{Prospects for diagnostic and therapeutic applications}

Amyloidoses are particularly difficult to diagnose. In the case of systemic amyloidoses, most of them are rare and the symptoms can be very broad and are often mimicked by more common disorders; the definitive diagnostic is currently established after analysis of biopsied tissues by Congo red binding and immunostaining. In the case of $A D$ and $P D$, such a definitive diagnosis has traditionally be made by post-mortem histological analysis of brain tissue although recent brain imaging have the potential to detect preclinical anatomic and functional changes as well as protein deposition in the brain [166,167]. Moreover, there is a body of evidence showing that protein deposition and irreparable damages occur many years before the significant symptoms appear [167]. Thus, the treatment of patients should ideally start before the apparition of symptoms and this requires novel diagnostic methods, preferably noninvasive, allowing an early detection of these diseases [156]. Ligands that are sensitive and selective for amyloid fibrils or their precursor species are of special interest to detect preclinical pathology. In this context, the use of conformational antibodies is of particular interest.

According to the amyloid cascade hypothesis, protein misfolding and aggregation are the key events in triggering the disease. Thus several therapeutic approaches targeting these phenomena are being considered including: (i) reducing the expression level of the amyloidogenic protein, (ii) increasing the clearance of the misfolded amyloidogenic proteins, (iii) increasing the stability of the properly folded amyloidogenic proteins, (iv) neutralising the cytotoxic species, and/or ( $v$ ) inhibiting or reversing the aggregation of misfolded proteins into oligomers and fibrils. A large body of studies, in addition to those described in the previous section, carried out both in vitro and in animal models has shown that antibodies or antibody fragments can, at least, mediate the last three effects $[21,163,168]$ and improve the cognitive deficit [163,169]. Despite these promising results obtained in the laboratory, clinical trials involving active (i.e. injection of the antigen to induce the production of antibodies) or passive (i.e. administration of specific antibodies) immunotherapies did not, however, lead to the expected effects [170]. These observations actually reflect, once more, the difficulty of predicting the effects of an antibody depending of the context; theories why these failures occurred include late stage disease treatment and poor penetration of antibody in the brain. Immunotherapy, especially to treat AD and PD, is nevertheless still a very active field of research for which several new clinical trials are ongoing $[170,171]$. Based on the results of previous works, recent studies are refining the selection of specific epitopes for active immunisation and developing strategies to increase the ability of antibodies to cross the BBB for passive immunisation [170].

In case of passive immunotherapy, the main challenges are the following: (i) How to deliver the antibodies where they need to act? (ii) In case of disease associated with intracellular aggregates and/ or aggregation in the brain, antibodies must be able to respectively penetrate into the cells and be active in the cellular environment and/or to cross the blood brain barrier. (iii) The antibody should not be toxic or immunogenic. The studies presented in the previous sections are a proof of concept that nanobodies can target each species formed on the pathway of fibril formation and interfere with each step of the aggregation process (Fig. 1). Some of these effects have even been shown in vivo. This clearly demonstrates the therapeutic potential of nanobodies. In the last section, we will further show that, due to their unique properties, nanobodies are promising to address the challenges mentioned above from both a diagnostic and therapeutic point of view.

\subsection{Nanobodies are efficiently produced intracellularly and can cross membranes and the $B B B$}

As mentioned above, in case of amyloidoses associated with intracellular aggregates and/or aggregation in the brain, antibodies must be able to respectively penetrate into the cells and be active in the cellular environment and/or to cross the BBB.

Antibodies that function inside the cells are called intrabodies. The function of conventional antibodies or antibody fragments inside the cell is generally compromised due to the inability of the disulphide bonds to form in the reducing environment of the cytoplasmic environment and thus the inability of the protein to fold or remain folded in its native state. Due to the high stability of nanobodies [37], their conserved disulphide bridge can in many cases be deleted without significantly affecting their functionality [71] and nanobodies fold well into functional entities in the reducing intracellular environment [172]. Given this intracellular robustness, combined to the easiness with which they can be fused to signal peptides to direct them to specific cellular compartments, nanobodies have been used for a variety of purposes in cells including imaging [172-174]. Another major obstacle to antibody applications in therapies is the delivery of the antibody at a sufficient concentration and to sufficient number of target cells for a sufficient time. Strategies to continuously deliver recombinant antibody fragments as genes is an increasing developing field and could easily be transferred to nanobodies [175].

Conventional antibodies cannot traverse cell membranes and are not able to significantly freely diffuse across BBB. Indeed, only $0.1-0.2 \%$ of circulating antibodies are found in brain at steady state concentrations [176]. In addition to the PrioV3 and ni3A described above, several other nanobodies able to cross the BBB have been reported [157,172,177-181]. The amount of transduced nanobodies as well as the mechanism by which they are transduced, varie significantly form one sequence to another. And despite the increased intake level, there is still a debate whether the transduced amount of nanobodies is sufficient to allow imaging and therapy [172,182]. Again, due to their small size, high stability and easiness to be engineered, the various approaches that have been reported to transduce proteins (i.e. coupling them to a shuttle peptide or protein to facilitate their passage through the BBB via receptor mediated transcytosis) should be easily transferable to nanobodies.

\subsection{Nanobodies as powerful imaging tools}

Given the high stability of nanobodies, a large number of labelling strategies are possible including the use of radionulei [183], 
near-infrared fluorophores [184] and fluorescent proteins [182]. Nanobodies fused to a fluorescent protein (e.g. mRFP and GFP), referred to as chromobodies, have been extensively used as a research tool to recognise and trace their specific antigen in different subcellular compartments [172,173]. Such chromobodies can be expressed throughout an organism allowing the localisation of their specific targets within the cell; this approach is of particular interest given the recent consideration in the amyloid field that aggregates of a series of proteins behave as prions and can move between cells [17]. Moreover, by coupling nanobodies that are specific to the different species formed upon fibril formation (i.e. misfolded monomers, oligomers and fibrils) to different fluorescent proteins, one should be able to follow simultaneous the evolution these species in time and space.

The critical features of an in vivo imaging agent are: stability in vivo, rapid reaching and interaction with its target, high target to background ratio, fast elimination of unbound molecules from the body, minimal non-specific accumulation in tissues surrounding the molecular target and low immunogenicity. These features are essentially all fulfilled by nanobodies $[185,186]$ and a series of studies suggest that nanobodies could become the next generation of magic bullets for immune-imaging $[182,187]$. For example, radiolabelled nanobodies that are specific to carcino embryonic antigen (i.e. the epidermal growth factor and the HER2 receptor expressed on cancer cells) show rapid (i.e. $1 \mathrm{~h}$ after injection) and specific uptake in mouse tumour model [187]. Due to the high specificity, high tissues penetrance and short biological half-life of the nanobody, imaging with sufficient contrast is possible shortly after its injection which, from a practical point of view, will be much appreciable for the patients. It also allows the use of short-lived, and thus less toxic, radionuclei [187].

\subsection{Immunogenicity and humanisation of nanobodies}

Non-human antibodies may trigger an immune reaction in humans, limiting their use, especially for prolonged therapeutic applications involving multiple administrations. Thus, non-human antibodies need to be "humanised" to be accepted as human therapeutics; that is their sequence has to be modified to increase their similarity to antibody variants naturally produced in humans. Since all nanobodies belong to the same sequence family which is closely related to the human $\mathrm{V}_{\mathrm{H}}$ of family III (i.e. they show $\sim 80 \%$ sequence homology with the human family III when comparing the framework regions [40]), they should exhibit low immunogenicity. And indeed, so far, no immune adverse events have been reported in clinical studies [33]. Moreover, if necessary, their humanisation (i.e. mutation of specific residues to their human $V_{H}$ equivalent) is rather straightforward since a nanobody differs from a human $\mathrm{V}_{\mathrm{H}}(\mathrm{i})$ in four hallmark nanobody-specific amino acids located in the framework 2 which normally interacts with the $\mathrm{V}_{\mathrm{L}}$ domain in conventional antibodies (positions 42, 49, 50 and 52) and (ii) in about 10 amino acids, outside the framework 2 , spread all over the surface of the nanobody. The group of S. Muyldermans has established two strategies to efficiently humanise nanobodies without significantly affecting their binding specificity, stability and solubility [188]. The first strategy consists in mutating the 10 surface residues and 2 nanobody-specific FR2 residues (positions 49 and 50 ). The second strategy consists in grafting the CDRs of the nanobody of interest onto a universal humanised nanobody scaffold. The latter has been designed using the first strategy (i.e. by mutating 10 surface residues and residues at positions 49 and 50) on cAb-BcII10, a nanobody which has very favourable properties including high stability, high yield of production [37], and a high tolerance to CDR grafting [72].

\section{Conclusions}

As summarised in Fig. 1, the results reported in this review clearly demonstrate that nanobodies, from different origins (i.e. from immune, non-immune or synthetic libraries) could target each species formed on the pathway of fibril formation. Moreover, their binding to their specific target can inhibit fibril formation at various stages ranging from the first step (i.e. inhibition of the formation of the amyloidogenic intermediate via the stabilisation of the native state) to the self-association of protofibrils. Importantly, when not adapted to the conditions used to trigger amyloid fibril formation, the properties of nanobodies can easily be improved by protein engineering. Moreover, manifold constructs, for example fusion to an enzyme or GFP, can readily be generated to enable easy imaging both in vitro and in vivo. These studies also demonstrate that nanobodies are unique structural probes. They can trap species that are only transiently populated (i.e. the aggregation nucleus) and they can be used as crystallisation chaperone to obtain, at high resolution, structural information about these species. Finally, they are able to target very sensitive/discrete differences between various populations of oligomeric species or amyloid fibrils which cannot be distinguished by standard techniques (i.e. TEM, FTIR, NMR). These studies highlight the importance of nanobodies as unique and powerful research tools to better understand the complexity of fibril formation and the associated toxicity both in vitro and in vivo, and thus to identify and validate drug targets. Given their small size, high stability, easiness to label and better cell and BBB penetration, nanobodies constitute valuable imaging tools. Again, due to their unique properties, nanobodies constitute an additional therapeutic approach for amyloidoses although the question of efficient delivery to the brain remains to be solved. The field of applications of nanobodies is expending at an impressive rate and transferring the knowledge gained form these studies, especially in terms of engineering, to the field of amyloidoses holds good premise for further developments of nanobodies to study, diagnose and eventually treat amyloidoses.

\section{Conflict of interest}

There is no conlict of interest.

\section{Acknowledgements}

We acknowledge fundings from Fonds de la Recherche Fondamentale et Collective (2.4581.12), Fonds de la Recherche Scientifique (FRS-FNRS, 1.C039.09 and MIS.4505.11), Fonds Spéciaux from the University of Liège (11/108) and the Belgian program of Interuniversity Attraction Poles administered by the Federal Office for Scientific Technical and Cultural Affairs (P7/44 and P6/19). We thank Jean-Marie Frère, Céline Huynen and Roya Barumandzadeh for critical reading of the manuscript and for many helpful suggestions.

\section{References}

[1] C.M. Dobson, Protein folding and misfolding, Nature 426 (2003) 884-890.

[2] A.V. Sorokin, E.R. Kim, L.P. Ovchinnikov, Proteasome system of protein degradation and processing, Biochemistry (Moscow) 74 (2009) 1411-1442.

[3] F. Chiti, C.M. Dobson, Protein misfolding, functional amyloid, and human disease, Annu. Rev. Biochem. 75 (2006) 333-366.

[4] C.M. Dobson, Principles of protein folding, misfolding and aggregation, Semin. Cell Dev. Biol. 15 (2004) 3-16.

[5] E.R. Dorsey, R. Constantinescu, J.P. Thompson, et al., Projected number of people with Parkinson disease in the most populous nations, 2005 through 2030, Neurology 68 (2007) 384-386.

[6] W. Thies, L. Bleiler, 2013 Alzheimer's disease facts and figures, Alzheimers Dement. 9 (2013) 208-245. 
[7] G.H. Suh, S.H. Ryu, D.W. Lee, et al., Cholinesterase inhibitors for Alzheimer disease: do they provide more than symptomatic benefits? Am. J. Geriatr. Psychiatry 19 (2011) 266-273.

[8] M.A. Brodsky, B.S. Park, J.G. Nutt, Effects of a dopamine agonist on the pharmacodynamics of levodopa in Parkinson disease, Arch. Neurol. 67 (2010) 27-32.

[9] M. Sunde, L.C. Serpell, M. Bartlam, et al., Common core structure of amyloid fibrils by synchrotron X-ray diffraction, J. Mol. Biol. 273 (1997) 729-739.

[10] M. Stefani, C.M. Dobson, Protein aggregation and aggregate toxicity: new insights into protein folding, misfolding diseases and biological evolution, J. Mol. Med. 81 (2003) 678-699.

[11] T. Guilliams, F. El-Turk, A.K. Buell, et al., Nanobodies raised against monomeric alpha-synuclein distinguish between fibrils at different maturation stages, J. Mol. Biol. 425 (2013) 2397-2411.

[12] M.F. Mossuto, A. Dhulesia, G. Devlin, et al., The non-core regions of human lysozyme amyloid fibrils influence cytotoxicity, J. Mol. Biol. 402 (2010) 783-796.

[13] J.T. Jarrett, P.T. Lansbury Jr., Seeding "one-dimensional crystallization" of amyloid: a pathogenic mechanism in Alzheimer's disease and scrapie? Cell 73 (1993) 1055-1058.

[14] S.I. Cohen, S. Linse, L.M. Luheshi, et al., Proliferation of amyloid-beta42 aggregates occurs through a secondary nucleation mechanism, Proc. Natl. Acad. Sci. U. S. A. 110 (2013) 9758-9763.

[15] J.S. Jeong, A. Ansaloni, R. Mezzenga, et al., Novel mechanistic insight into the molecular basis of amyloid polymorphism and secondary nucleation during amyloid formation, J. Mol. Biol. 425 (2013) 1765-1781.

[16] G. Ramachandran, J.B. Udgaonkar, Evidence for the existence of a secondary pathway for fibril growth during the aggregation of tau, J. Mol. Biol. 421 (2012) 296-314.

[17] M. Costanzo, C. Zurzolo, The cell biology of prion-like spread of protein aggregates: mechanisms and implication in neurodegeneration, Biochem. J. 452 (2013) 1-17.

[18] M.A. Speed, T. Morshead, D.I. Wang, et al., Conformation of P22 tailspike folding and aggregation intermediates probed by monoclonal antibodies, Protein Sci. 6 (1997) 99-108.

[19] B. O'Nuallain, R. Wetzel, Conformational Abs recognizing a generic amyloid fibril epitope, Proc. Natl. Acad. Sci. U. S. A. 99 (2002) 1485-1490.

[20] B. O'Nuallain, I. Klyubin, J.M. Mc Donald, et al., A monoclonal antibody against synthetic Abeta dimer assemblies neutralizes brain-derived synaptic plasticity-disrupting Abeta, J. Neurochem. 119 (2011) 189-201.

[21] M. Dumoulin, C.M. Dobson, Probing the origins, diagnosis and treatment of amyloid diseases using antibodies, Biochimie 86 (2004) 589-600.

[22] F. Eghiaian, J. Grosclaude, S. Lesceu, et al., Insight into the $\operatorname{PrP}^{\mathrm{C}}->\mathrm{PrP}^{\mathrm{Sc}}$ conversion from the structures of antibody-bound ovine prion scrapiesusceptibility variants, Proc. Natl. Acad. Sci. U. S. A. 101 (2004) 10254-10259.

[23] R. Kayed, E. Head, F. Sarsoza, et al., Fibril specific, conformation dependent antibodies recognize a generic epitope common to amyloid fibrils and fibrillar oligomers that is absent in prefibrillar oligomers, Mol. Neurodegener. 2 (2007) 18.

[24] C. Haupt, M. Bereza, S.T. Kumar, et al., Pattern recognition with a fibrilspecific antibody fragment reveals the surface variability of natural amyloid fibrils, J. Mol. Biol. 408 (2011) 529-540.

[25] H. Arai, C. Glabe, H. Luecke, Crystal structure of a conformation-dependent rabbit IgG Fab specific for amyloid prefibrillar oligomers, Biochim. Biophys. Acta 1820 (2012) 1908-1914.

[26] M. Tayebi, D.R. Jones, W.A. Taylor, et al., PrP(Sc)-specific antibodies with the ability to immunodetect prion oligomers, PLoS ONE 6 (2011) e19998.

[27] E.J. De Genst, T. Guilliams, J. Wellens, et al., Structure and properties of a complex of alpha-synuclein and a single-domain camelid antibody, J. Mol. Biol. 402 (2010) 326-343.

[28] G. Goldsteins, H. Persson, K. Andersson, et al., Exposure of cryptic epitopes on transthyretin only in amyloid and in amyloidogenic mutants, Proc. Natl. Acad. Sci. U. S. A. 96 (1999) 3108-3113.

[29] K. Manoutcharian, G. Acero, M.E. Munguia, et al., Amyloid-beta peptidespecific single chain Fv antibodies isolated from an immune phage display library, J. Neuroimmunol. 145 (2003) 12-17.

[30] Z.F. Zhao, G.Q. Gao, S. Liu, et al., Screening for a human single chain Fv antibody against epitope on amyloid-beta 1-40 from a human phage display library, Chin. Med. J. (Engl.) 120 (2007) 1931-1934.

[31] M. Medecigo, K. Manoutcharian, V. Vasilevko, et al., Novel amyloid-beta specific scFv and VH antibody fragments from human and mouse phage display antibody libraries, J. Neuroimmunol. 223 (2010) 104-114.

[32] C. Hamers-Casterman, T. Atarhouch, S. Muyldermans, et al., Naturally occurring antibodies devoid of light chains, Nature 363 (1993) 446-448.

[33] F. Van Bockstaele, J.B. Holz, H. Revets, The development of nanobodies for therapeutic applications, Curr. Opin. Investig. Drugs 10 (2009) 1212-1224.

[34] S. Muyldermans, Single domain camel antibodies: current status, J. Biotechnol. 74 (2001) 277-302.

[35] R. van der Linden, B. de Geus, W. Stok, et al., Induction of immune responses and molecular cloning of the heavy chain antibody repertoire of Lama glama, J. Immunol. Methods 240 (2000) 185-195.

[36] M. Lauwereys, M. Arbabi Ghahroudi, A. Desmyter, et al., Potent enzyme inhibitors derived from dromedary heavy-chain antibodies, EMBO J. 17 (1998) $3512-3520$
[37] M. Dumoulin, K. Conrath, A. Van Meirhaeghe, et al., Single-domain antibody fragments with high conformational stability, Protein Sci. 11 (2002) $500-515$

[38] M.P. Lefranc, F. Ehrenmann, C. Ginestoux, et al., Use of IMGT((R)) databases and tools for antibody engineering and humanization, Methods Mol. Biol. 907 (2012) 3-37.

[39] K.E. Conrath, U. Wernery, S. Muyldermans, et al., Emergence and evolution of functional heavy-chain antibodies in Camelidae, Dev. Comp. Immunol. 27 (2003) 87-103.

[40] S. Muyldermans, C. Cambillau, L. Wyns, Recognition of antigens by singledomain antibody fragments: the superfluous luxury of paired domains, Trends Biochem. Sci. 26 (2001) 230-235.

[41] S. Muyldermans, T. Atarhouch, J. Saldanha, et al., Sequence and structure of VH domain from naturally occurring camel heavy chain immunoglobulins lacking light chains, Protein Eng. 7 (1994) 1129-1135.

[42] K.B. Vu, M.A. Ghahroudi, L. Wyns, et al., Comparison of llama VH sequences from conventional and heavy chain antibodies, Mol. Immunol. 34 (1997) 1121-1131.

[43] T.T. Wu, G. Johnson, E.A. Kabat, Length distribution of CDRH3 in antibodies, Proteins 16 (1993) $1-7$.

[44] P.H. Chan, E. Pardon, L. Menzer, et al., Engineering a camelid antibody fragment that binds to the active site of human lysozyme and inhibits its conversion into amyloid fibrils, Biochemistry 47 (2008) 11041-11054.

[45] A. Desmyter, T.R. Transue, M.A. Ghahroudi, et al., Crystal structure of a camel single-domain VH antibody fragment in complex with lysozyme, Nat. Struct. Biol. 3 (1996) 803-811.

[46] S. Muyldermans, Nanobodies: natural single-domain antibodies, Annu. Rev. Biochem. 82 (2013) 775-797.

[47] K. Decanniere, S. Muyldermans, L. Wyns, Canonical antigen-binding loop structures in immunoglobulins: more structures, more canonical classes? J. Mol. Biol. 300 (2000) 83-91.

[48] E. De Genst, K. Silence, K. Decanniere, et al., Molecular basis for the preferential cleft recognition by dromedary heavy-chain antibodies, Proc. Natl. Acad. Sci. U. S. A. 103 (2006) 4586-4591.

[49] A. Desmyter, S. Spinelli, F. Payan, et al., Three camelid $\mathrm{V}_{\mathrm{H}} \mathrm{H}$ domains in complex with porcine pancreatic alpha-amylase. Inhibition and versatility of binding topology, J. Biol. Chem. 277 (2002) 23645-23650.

[50] J. Wesolowski, V. Alzogaray, J. Reyelt, et al., Single domain antibodies: promising experimental and therapeutic tools in infection and immunity, Med. Microbiol. Immunol. 198 (2009) 157-174.

[51] T.R. Transue, E. De Genst, M.A. Ghahroudi, et al., Camel single-domain antibody inhibits enzyme by mimicking carbohydrate substrate, Proteins 32 (1998) 515-522.

[52] A. Marquardt, S. Muyldermans, M. Przybylski, A synthetic camel antilysozyme peptide antibody (peptibody) with flexible loop structure identified by high-resolution affinity mass spectrometry, Chemistry 12 (2006) 1915-1923.

[53] J. Govaert, M. Pellis, N. Deschacht, et al., Dual beneficial effect of interloop disulfide bond for single domain antibody fragments, J. Biol. Chem. 287 (2012) 1970-1979.

[54] V.K. Nguyen, R. Hamers, L. Wyns, et al., Camel heavy-chain antibodies: diverse germline $\mathrm{V}(\mathrm{H}) \mathrm{H}$ and specific mechanisms enlarge the antigenbinding repertoire, EMBO J. 19 (2000) 921-930.

[55] G. Wei, W. Meng, H. Guo, et al., Potent neutralization of influenza A virus by a single-domain antibody blocking M2 ion channel protein, PLoS ONE 6 (2011) e28309.

[56] J. Yan, G. Li, Y. Hu, et al., Construction of a synthetic phage-displayed nanobody library with CDR3 regions randomized by trinucleotide cassettes for diagnostic applications, J. Transl. Med. 12 (2014) 343.

[57] F. Fleetwood, N. Devoogdt, M. Pellis, et al., Surface display of a single-domain antibody library on gram-positive bacteria, Cell. Mol. Life Sci. 70 (2013) $1081-1093$.

[58] J. Dong, A.A. Thompson, Y. Fan, et al., A single-domain llama antibody potently inhibits the enzymatic activity of botulinum neurotoxin by binding to the non-catalytic alpha-exosite binding region, J. Mol. Biol. 397 (2010) $1106-1118$.

[59] C. Perruchini, F. Pecorari, J.P. Bourgeois, et al., Llama VHH antibody fragments against GFAP: better diffusion in fixed tissues than classical monoclonal antibodies, Acta Neuropathol. 118 (2009) 685-695.

[60] F. Rahbarizadeh, M.J. Rasaee, M. Forouzandeh-Moghadam, et al. High expression and purification of the recombinant camelid anti-MUC1 single domain antibodies in Escherichia coli, Protein Expr. Purif. 44 (2005) $32-38$.

[61] H. Bakherad, S.L. Mousavi Gargari, I. Rasooli, et al., In vivo neutralization of botulinum neurotoxins serotype $\mathrm{E}$ with heavy-chain camelid antibodies $\left(\mathrm{V}_{\mathrm{H}} \mathrm{H}\right)$, Mol. Biotechnol. 55 (2013) 159-167.

[62] L.G. Frenken, R.H. van der Linden, P.W. Hermans, et al., Isolation of antigen specific llama VHH antibody fragments and their high level secretion by Saccharomyces cerevisiae, J. Biotechnol. 78 (2000) 11-21.

[63] A. Gorlani, H. de Haard, T. Verrips, Expression of VHHs in Saccharomyces cerevisiae, Methods Mol. Biol. 911 (2012) 277-286.

[64] S. De Buck, V. Virdi, T. De Meyer, et al., Production of camel-like antibodies in plants, Methods Mol. Biol. 911 (2012) 305-324.

[65] Y.H. Teh, T.A. Kavanagh, High-level expression of Camelid nanobodies in Nicotiana benthamiana, Transgenic Res. 19 (2010) 575-586. 
[66] F. Rahbarizadeh, D. Ahmadvand, Z. Sharifzadeh, Nanobody; an old concept and new vehicle for immunotargeting. Immunol. Invest. 40 (2011) 299-338.

[67] M. Arbabi Ghahroudi, A. Desmyter, L. Wyns, et al., Selection and identification of single domain antibody fragments from camel heavy-chain antibodies, FEBS Lett. 414 (1997) 521-526.

[68] K. Conrath, M. Lauwereys, L. Wyns, et al., Camel single-domain antibodies as modular building units in bispecific and bivalent antibody constructs, J. Biol. Chem. 276 (2001) 7346-7350.

[69] M. Dumoulin, A.M. Last, A. Desmyter, et al., A camelid antibody fragment inhibits the formation of amyloid fibrils by human lysozyme, Nature 424 (2003) 783-788.

[70] Y. Hagihara, S. Mine, K. Uegaki, Stabilization of an immunoglobulin fold domain by an engineered disulfide bond at the buried hydrophobic region, J. Biol. Chem. 282 (2007) 36489-36495.

[71] D. Saerens, K. Conrath, J. Govaert, et al., Disulfide bond introduction for general stabilization of immunoglobulin heavy-chain variable domains, J. Mol. Biol. 377 (2008) 478-488.

[72] D. Saerens, M. Pellis, R. Loris, et al., Identification of a universal VHH framework to graft non-canonical antigen-binding loops of camel singledomain antibodies, J. Mol. Biol. 352 (2005) 597-607.

[73] P. Vanlandschoot, C. Stortelers, E. Beirnaert, et al., Nanobodies(R): new ammunition to battle viruses, Antivir. Res. 92 (2011) 389-407.

[74] M. Dumoulin, J.R. Kumita, C.M. Dobson, Normal and aberrant biological selfassembly: insights from studies of human lysozyme and its amyloidogenic variants, Acc. Chem. Res. 39 (2006) 603-610.

[75] S. Girnius, M. Skinner, B. Spencer, et al., A new lysozyme tyr54asn mutation causing amyloidosis in a family of Swedish ancestry with gastrointestinal symptoms, Amyloid 19 (2012) 182-185.

[76] M. Dumoulin, R.J.K. Johnson, V. Bellotti, C. Dobson, Human lysozyme amyloidosis, in: V. Uversky, A.L. Fink (Eds.), Protein Misfolding, Aggregation and Conformational Diseases. II. Molecular Basis of Conformational Diseases Vol. II, K. A. P., Dordrecht (The Netherlands), 2007, pp. 285-308.

[77] M. Dumoulin, Familial amyloidosis caused by lysozyme mutations, in: J.W.K. Marina Ramirez-Alvarado, C.M. Dobson (Eds.), Protein Misfolding Diseases: Basis of Protein Misfolding, Pathophysiology, Current, and Emerging Therapies, John Wileys and Sons, Inc., Hoboken, New Jersy, USA, 2010, pp. 867-884.

[78] S. Reitamo, M. Klockars, M. Adinolfi, et al., Human lysozyme (origin and distribution in health and disease), Ric. Clin. Lab. 8 (1978) 211-231.

[79] D.R. Booth, M. Sunde, V. Bellotti, et al., Instability, unfolding and aggregation of human lysozyme variants underlying amyloid fibrillogenesis, Nature 385 (1997) 787-793.

[80] R.J. Johnson, J. Christodoulou, M. Dumoulin, et al., Rationalising lysozyme amyloidosis: insights from the structure and solution dynamics of T70N lysozyme, J. Mol. Biol. 352 (2005) 823-836.

[81] D. Canet, A.M. Last, P. Tito, et al., Local cooperativity in the unfolding of an amyloidogenic variant of human lysozyme, Nat. Struct. Biol. 9 (2002)308-315.

[82] M. Dumoulin, D. Canet, A.M. Last, et al., Reduced global cooperativity is a common feature underlying the amyloidogenicity of pathogenic lysozyme mutations, J. Mol. Biol. 346 (2005) 773-788.

[83] E.J. De Genst, P.H. Chan, E. Pardon, et al., A nanobody binding to nonamyloidogenic regions of the protein human lysozyme enhances partial unfolding but inhibits amyloid fibril formation, J. Phys. Chem. B 117 (2013) 13245-13258.

[84] S. Valleix, J.D. Gillmore, F. Bridoux, et al., Hereditary systemic amyloidosis due to Asp76Asn variant beta2-microglobulin, N. Engl. J. Med. 366 (2012) $2276-2283$.

[85] R.P. Linke, H. Hampl, H. Lobeck, et al., Lysine-specific cleavage of beta 2 microglobulin in amyloid deposits associated with hemodialysis, Kidney Int. 36 (1989) 675-681.

[86] V. Bellotti, M. Stoppini, P. Mangione, et al., Beta2-microglobulin can be refolded into a native state from ex vivo amyloid fibrils, Eur. J. Biochem. 258 (1998) 61-67.

[87] H.L. Ploegh, H.T. Orr, J.L. Stominger, Biosynthesis and cell surface localization of nonglycosylated human histocompatibility antigens, J. Immunol. 126 (1981) 270-275.

[88] P.J. Bjorkman, M.A. Saper, B. Samraoui, et al., Structure of the human class I histocompatibility antigen, HLA-A2, Nature 329 (1987) 506-512.

[89] C.H. Trinh, D.P. Smith, A.P. Kalverda, et al., Crystal structure of monomeric human beta-2-microglobulin reveals clues to its amyloidogenic properties, Proc. Natl. Acad. Sci. U. S. A. 99 (2002) 9771-9776.

[90] T. Eichner, S.E. Radford, Understanding the complex mechanisms of beta2microglobulin amyloid assembly, FEBS J. 278 (2011) 3868-3883.

[91] G. Esposito, A. Corazza, V. Bellotti, Pathological self-aggregation of beta(2)microglobulin: a challenge for protein biophysics, Subcell. Biochem. 65 (2012) 165-183.

[92] T. Eichner, S.E. Radford, A generic mechanism of beta2-microglobulin amyloid assembly at neutral $\mathrm{pH}$ involving a specific proline switch, J. Mol. Biol. 386 (2009) 1312-1326.

[93] C. Santambrogio, S. Ricagno, M. Colombo, et al., DE-loop mutations affect beta2 microglobulin stability, oligomerization, and the low-pH unfolded form, Protein Sci. 19 (2010) 1386-1394.

[94] K. Domanska, S. Vanderhaegen, V. Srinivasan, et al., Atomic structure of a nanobody-trapped domain-swapped dimer of an amyloidogenic beta2microglobulin variant, Proc. Natl. Acad. Sci. U. S. A. 108 (2011) 1314-1319.
[95] G. Esposito, R. Michelutti, G. Verdone, et al., Removal of the N-termina hexapeptide from human beta2-microglobulin facilitates protein aggregation and fibril formation, Protein Sci. 9 (2000) 831-845.

[96] M.I. Ivanova, M.J. Thompson, D. Eisenberg, A systematic screen of beta(2)microglobulin and insulin for amyloid-like segments, Proc. Natl. Acad. Sci. U. S. A. 103 (2006) 4079-4082.

[97] S. Vanderhaegen, M. Fislage, K. Domanska, et al., Structure of an early nativelike intermediate of beta2-microglobulin amyloidogenesis, Protein Sci. 22 (2013) 1349-1357.

[98] T. Pringsheim, N. Jette, A. Frolkis, et al., The prevalence of Parkinson's disease: a systematic review and meta-analysis, Mov. Disord. 29 (2014) $1583-1590$

[99] N. Malek, D. Swallow, K.A. Grosset, et al., Alpha-synuclein in peripheral tissues and body fluids as a biomarker for Parkinson's disease - a systematic review, Acta Neurol. Scand. 130 (2014) 59-72.

[100] V.N. Uversky, J. Li, P. Souillac, et al., Biophysical properties of the synucleins and their propensities to fibrillate: inhibition of alpha-synuclein assembly by beta- and gamma-synucleins, J. Biol. Chem. 277 (2002) 11970-11978.

[101] J.M. George, The synucleins, Genome Biol. 3 (2002). REVIEWS3002.

[102] H.A. Lashuel, C.R. Overk, A. Oueslati, et al., The many faces of alphasynuclein: from structure and toxicity to therapeutic target, Nat. Rev. Neurosci. 14 (2013) 38-48.

[103] N.M. Bonini, B.I. Giasson, Snaring the function of alpha-synuclein, Cell 123 (2005) 359-361.

[104] J. Burre, M. Sharma, T. Tsetsenis, et al., Alpha-synuclein promotes SNARE complex assembly in vivo and in vitro, Science 329 (2010) 1663-1667.

[105] M. Kasten, C. Klein, The many faces of alpha-synuclein mutations, Mov. Disord. 28 (2013) 697-701.

[106] K. Beyer, Alpha-synuclein structure, posttranslational modification and alternative splicing as aggregation enhancers, Acta Neuropathol. 112 (2006) 237-251.

[107] W. Wang, I. Perovic, J. Chittuluru, et al., A soluble alpha-synuclein construct forms a dynamic tetramer, Proc. Natl. Acad. Sci. U. S. A. 108 (2011) 17797-17802.

[108] B.A. Silva, L. Breydo, V.N. Uversky, Targeting the chameleon: a focused look at alpha-synuclein and its roles in neurodegeneration, Mol. Neurobiol. 47 (2013) 446-459.

[109] H.J. Koo, M.Y. Choi, H. Im, Aggregation-defective alpha-synuclein mutants inhibit the fibrillation of Parkinson's disease-linked alpha-synuclein variants, Biochem. Biophys. Res. Commun. 386 (2009) 165-169.

[110] J. Meuvis, M. Gerard, L. Desender, et al., The conformation and the aggregation kinetics of alpha-synuclein depend on the proline residues in its Cterminal region, Biochemistry 49 (2010) 9345-9352.

[111] C.R. Bodner, A.S. Maltsev, C.M. Dobson, et al., Differential phospholipid binding of alpha-synuclein variants implicated in Parkinson's disease revealed by solution NMR spectroscopy, Biochemistry 49 (2010) 862-871.

[112] H.Y. Kim, M.K. Cho, A. Kumar, et al., Structural properties of pore-forming oligomers of alpha-synuclein, J. Am. Chem. Soc. 131 (2009) 17482-17489.

[113] Z. Qin, D. Hu, S. Han, et al., Role of different regions of alpha-synuclein in the assembly of fibrils, Biochemistry 46 (2007) 13322-13330.

[114] L. Solforosi, M. Milani, N. Mancini, et al., A closer look at prion strains: characterization and important implications, Prion 7 (2013) 99-108.

[115] S.K. Kaufman, M.I. Diamond, Prion-like propagation of protein aggregation and related therapeutic strategies, Neurotherapeutics 10 (2013) $371-382$.

[116] A. Thompson, A. MacKay, P. Rudge, et al., Behavioral and psychiatric symptoms in prion disease, Am. J. Psychiatry 171 (2014) 265-274.

[117] A. Ladogana, M. Puopolo, E.A. Croes, et al., Mortality from Creutzfeldt-Jakob disease and related disorders in Europe, Australia, and Canada, Neurology 64 (2005) 1586-1591.

[118] L.T. Takada, M.D. Geschwind, Prion diseases, Semin. Neurol. 33 (2013) $348-356$.

[119] A. Kraus, B.R. Groveman, B. Caughey, Prions and the potential transmissibility of protein misfolding diseases, Annu. Rev. Microbiol. 67 (2013) 543-564.

[120] K.M. Pan, M. Baldwin, J. Nguyen, et al., Conversion of alpha-helices into betasheets features in the formation of the scrapie prion proteins, Proc. Natl. Acad. Sci. U. S. A. 90 (1993) 10962-10966.

[121] M.W. Brazier, A.I. Mot, A.R. White, et al., Immunotherapeutic approaches in prion disease: progress, challenges and potential directions, Ther. Deliv. 4 (2013) 615-628.

[122] R. Zahn, A. Liu, T. Luhrs, et al., NMR solution structure of the human prion protein, Proc. Natl. Acad. Sci. U. S. A. 97 (2000) 145-150.

[123] A. Andreeva, D. Howorth, J.M. Chandonia, et al., Data growth and its impact on the SCOP database: new developments, Nucleic Acids Res. 36 (2008) D419-D425.

[124] G. Forloni, N. Angeretti, R. Chiesa, et al., Neurotoxicity of a prion protein fragment, Nature 362 (1993) 543-546.

[125] R.N. Abskharon, G. Giachin, A. Wohlkonig, et al., Probing the N-terminal beta-sheet conversion in the crystal structure of the human prion protein bound to a nanobody, J. Am. Chem. Soc. 136 (2014) 937-944.

[126] D.R. Jones, W.A. Taylor, C. Bate, et al., A camelid anti-PrP antibody abrogates PrP replication in prion-permissive neuroblastoma cell lines, PLoS ONE 5 (2010) e9804. 
[127] M.A. David, D.R. Jones, M. Tayebi, Potential candidate camelid antibodies for the treatment of protein-misfolding diseases, J. Neuroimmunol. 272 (2014) $76-85$.

[128] A. Abu-Baker, G.A. Rouleau, Oculopharyngeal muscular dystrophy: recent advances in the understanding of the molecular pathogenic mechanisms and treatment strategies, Biochim. Biophys. Acta 1772 (2007) 173-185.

[129] A. Banerjee, L.H. Apponi, G.K. Pavlath, et al., PABPN1: molecular function and muscle disease, FEBS J. 280 (2013) 4230-4250.

[130] A. Chartier, B. Benoit, M. Simonelig, A Drosophila model of oculopharyngeal muscular dystrophy reveals intrinsic toxicity of PABPN1, EMBO J. 25 (2006) 2253-2262.

[131] X. Fan, P. Dion, J. Laganiere, et al., Oligomerization of polyalanine expanded PABPN1 facilitates nuclear protein aggregation that is associated with cel death, Hum. Mol. Genet. 10 (2001) 2341-2351.

[132] N. Scarafone, C. Pain, A. Fratamico, et al., Amyloid-like fibril formation by polyQ proteins: a critical balance between the polyQ length and the constraints imposed by the host protein, PLoS ONE 7 (2012) e31253.

[133] V. Raz, T. Abraham, E.W. van Zwet, et al., Reversible aggregation of PABPN1 pre-inclusion structures, Nucleus 2 (2011) 208-218.

[134] P. Verheesen, A. de Kluijver, S. van Koningsbruggen, et al., Prevention of oculopharyngeal muscular dystrophy-associated aggregation of nuclear polyA-binding protein with a single-domain intracellular antibody, Hum. Mol. Genet. 15 (2006) 105-111.

[135] A. Impagliazzo, A.W. Tepper, T.C. Verrips, et al., Structural basis for a PABPN1 aggregation-preventing antibody fragment in OPMD, FEBS Lett. 584 (2010) 1558-1564.

[136] A. Chartier, V. Raz, E. Sterrenburg, et al., Prevention of oculopharyngeal muscular dystrophy by muscular expression of Llama single-chain intrabodies in vivo, Hum. Mol. Genet. 18 (2009) 1849-1859.

[137] D.M. Walsh, I. Klyubin, J.V. Fadeeva, et al., Amyloid-beta oligomers: their production, toxicity and therapeutic inhibition, Biochem. Soc. Trans. 30 (2002) 552-557.

[138] J. Hardy, D.J. Selkoe, The amyloid hypothesis of Alzheimer's disease: progress and problems on the road to therapeutics, Science 297 (2002) 353-356.

[139] D.S. Wang, D.W. Dickson, J.S. Malter, Tissue transglutaminase, protein crosslinking and Alzheimer's disease: review and views, Int. J. Clin. Exp. Pathol. 1 (2008) 5-18.

[140] D.M. Walsh, A.M. Minogue, C. Sala Frigerio, et al., The APP family of proteins: similarities and differences, Biochem. Soc. Trans. 35 (2007) 416-420.

[141] D.W. Banner, B. Gsell, J. Benz, et al., Mapping the conformational space accessible to BACE2 using surface mutants and cocrystals with Fab fragments, Fynomers and Xaperones, Acta Crystallogr. D Biol. Crystallogr. 69 (2013) 1124-1137.

[142] T. Sato, N. Dohmae, Y. Qi, et al., Potential link between amyloid beta-protein 42 and C-terminal fragment gamma 49-99 of beta-amyloid precursor protein, J. Biol. Chem. 278 (2003) 24294-24301.

[143] B. De Strooper, W. Annaert, Novel research horizons for presenilins and gamma-secretases in cell biology and disease, Annu. Rev. Cell Dev. Biol. 26 (2010) 235-260.

[144] T. Iwatsubo, A. Odaka, N. Suzuki, et al., Visualization of A beta 42(43) and A beta 40 in senile plaques with end-specific A beta monoclonals: evidence that an initially deposited species is A beta 42(43), Neuron 13 (1994) 45-53.

[145] F. Kamenetz, T. Tomita, H. Hsieh, et al., APP processing and synaptic function, Neuron 37 (2003) 925-937.

[146] D.M. Walsh, D.J. Selkoe, A beta oligomers - a decade of discovery, J. Neurochem. 101 (2007) 1172-1184.

[147] I. Kuperstein, K. Broersen, I. Benilova, et al., Neurotoxicity of Alzheimer's disease Abeta peptides is induced by small changes in the Abeta42 to Abeta40 ratio, EMBO J. 29 (2010) 3408-3420.

[148] L. Wu, P. Rosa-Neto, G.Y. Hsiung, et al., Early-onset familial Alzheimer's disease (EOFAD), Can. J. Neurol. Sci. 39 (2012) 436-445.

[149] S.S. Sisodia, P.H. St George-Hyslop, gamma-Secretase, Notch, Abeta and Alzheimer's disease: where do the presenilins fit in? Nat. Rev. Neurosci. 3 (2002) 281-290.

[150] D. Lambracht-Washington, R.N. Rosenberg, Active DNA Abeta42 vaccination as immunotherapy for Alzheimer disease, Transl. Neurosci. 3 (2012) 307-313.

[151] C.E. Munte, M. Beck Erlach, W. Kremer, et al., Distinct conformational states of the Alzheimer beta-amyloid peptide can be detected by high-pressure NMR spectroscopy, Angew. Chem. Int. Ed. Engl. 52 (2013) 8943-8947.

[152] P. Lafaye, I. Achour, P. England, et al., Single-domain antibodies recognize selectively small oligomeric forms of amyloid beta, prevent Abeta-induced neurotoxicity and inhibit fibril formation, Mol. Immunol. 46 (2009) 695-704.

[153] C. Wu, M.T. Bowers, J.E. Shea, Molecular structures of quiescently grown and brain-derived polymorphic fibrils of the Alzheimer amyloid abeta9-40 peptide: a comparison to agitated fibrils, PLoS Comput. Biol. 6 (2010) e1000693.

[154] G. Habicht, C. Haupt, R.P. Friedrich, et al., Directed selection of a conformational antibody domain that prevents mature amyloid fibril formation by stabilizing Abeta protofibrils, Proc. Natl. Acad. Sci. U. S. A. 104 (2007) 19232-19237.

[155] I. Morgado, K. Wieligmann, M. Bereza, et al., Molecular basis of beta-amyloid oligomer recognition with a conformational antibody fragment, Proc. Natl Acad. Sci. U. S. A. 109 (2012) 12503-12508.
[156] K.S. Rutgers, A. van Remoortere, M.A. van Buchem, et al., Differential recognition of vascular and parenchymal beta amyloid deposition, Neurobiol. Aging 32 (2011) 1774-1783.

[157] K.S. Rutgers, R.J. Nabuurs, S.A. van den Berg, et al., Transmigration of beta amyloid specific heavy chain antibody fragments across the in vitro bloodbrain barrier, Neuroscience 190 (2011) 37-42.

[158] C. Haupt, I. Morgado, S.T. Kumar, et al., Amyloid fibril recognition with the conformational B10 antibody fragment depends on electrostatic interactions, J. Mol. Biol. 405 (2011) 341-348.

[159] J. Wacker, R. Ronicke, M. Westermann, et al., Oligomer-targeting with a conformational antibody fragment promotes toxicity in Abeta-expressing flies, Acta Neuropathol. Commun. 2 (2014) 43.

[160] P.N. Lacor, M.C. Buniel, L. Chang, et al., Synaptic targeting by Alzheimer'srelated amyloid beta oligomers, J. Neurosci. 24 (2004) 10191-10200.

[161] M.P. Lambert, P.T. Velasco, L. Chang, et al., Monoclonal antibodies that target pathological assemblies of Abeta, J. Neurochem. 100 (2007) 23-35.

[162] E.B. Lee, L.Z. Leng, B. Zhang, et al., Targeting amyloid-beta peptide (Abeta) oligomers by passive immunization with a conformation-selective monoclonal antibody improves learning and memory in Abeta precursor protein (APP) transgenic mice, J. Biol. Chem. 281 (2006) 4292-4299.

[163] F. Bard, R. Barbour, C. Cannon, et al., Epitope and isotype specificities of antibodies to beta-amyloid peptide for protection against Alzheimer's disease-like neuropathology, Proc. Natl. Acad. Sci. U. S. A. 100 (2003) 2023-2028.

[164] Y. Yan, C. Wang, Abeta42 is more rigid than Abeta40 at the C terminus: implications for Abeta aggregation and toxicity, J. Mol. Biol. 364 (2006) $853-862$.

[165] B. Kieninger, Z. Gioeva, S. Kruger, et al., PTAA and B10: new approaches to amyloid detection in tissue-evaluation of amyloid detection in tissue with a conjugated polyelectrolyte and a fibril-specific antibody fragment, Amyloid 18 (2011) 47-52.

[166] H. Chertkow, S. Black, Imaging biomarkers and their role in dementia clinical trials, Can. J. Neurol. Sci. 34 (Suppl. 1) (2007) S77-S83.

[167] R.J. Bateman, C. Xiong, T.L. Benzinger, et al., Clinical and biomarker changes in dominantly inherited Alzheimer's disease, N. Engl. J. Med. 367 (2012) $795-804$.

[168] D. Schenk, R. Barbour, W. Dunn, et al., Immunization with amyloid-beta attenuates Alzheimer-disease-like pathology in the PDAPP mouse, Nature 400 (1999) 173-177.

[169] J.C. Dodart, K.R. Bales, K.S. Gannon, et al., Immunization reverses memory deficits without reducing brain Abeta burden in Alzheimer's disease model, Nat. Neurosci. 5 (2002) 452-457.

[170] B. Spencer, E. Masliah, Immunotherapy for Alzheimer's disease: past, present and future, Front. Aging Neurosci. 6 (2014) 114.

[171] A. Cardinale, D. Merlo, P. Giunchedi, et al., Therapeutic application of intrabodies against age-related neurodegenerative disorders, Curr. Pharm. Des. 20 (2014) 6028-6036.

[172] T. Li, J.P. Bourgeois, S. Celli, et al., Cell-penetrating anti-GFAP VHH and corresponding fluorescent fusion protein VHH-GFP spontaneously cross the blood-brain barrier and specifically recognize astrocytes: application to brain imaging, FASEB J. 26 (2012) 3969-3979.

[173] U. Rothbauer, K. Zolghadr, S. Tillib, et al., Targeting and tracing antigens in live cells with fluorescent nanobodies, Nat. Methods 3 (2006) $887-889$.

[174] I. Van Audenhove, K. Van Impe, D. Ruano-Gallego, et al., Mapping cytoskeletal protein function in cells by means of nanobodies, Cytoskeleton (Hoboken) 70 (2013) 604-622.

[175] W.J. Bowers, X.O. Breakefield, M. Sena-Esteves, Genetic therapy for the nervous system, Hum. Mol. Genet. 20 (2011) R28-R41.

[176] J.F. Poduslo, G.L. Curran, C.T. Berg, Macromolecular permeability across the blood-nerve and blood-brain barriers, Proc. Natl. Acad. Sci. U. S. A. 91 (1994) 5705-5709.

[177] A. Muruganandam, J. Tanha, S. Narang, et al., Selection of phage-displayed llama single-domain antibodies that transmigrate across human bloodbrain barrier endothelium, FASEB J. 16 (2002) 240-242.

[178] A. Abulrob, H. Sprong, P. Van Bergen en Henegouwen, et al., The blood-brain barrier transmigrating single domain antibody: mechanisms of transport and antigenic epitopes in human brain endothelial cells, J. Neurochem. 95 (2005) 1201-1214.

[179] G. Caljon, V. Caveliers, T. Lahoutte, et al., Using microdialysis to analyse the passage of monovalent nanobodies through the blood-brain barrier, Br. J. Pharmacol. 165 (2012) 2341-2353.

[180] R.J. Nabuurs, K.S. Rutgers, M.M. Welling, et al., In vivo detection of amyloidbeta deposits using heavy chain antibody fragments in a transgenic mouse model for Alzheimer's disease, PLoS ONE 7 (2012) e38284.

[181] A.S. Haqqani, N. Caram-Salas, W. Ding, et al., Multiplexed evaluation of serum and CSF pharmacokinetics of brain-targeting single-domain antibodies using a NanoLC-SRM-ILIS method, Mol. Pharm. 10 (2013) $1542-1556$.

[182] J. De Vos, N. Devoogdt, T. Lahoutte, et al., Camelid single-domain antibodyfragment engineering for (pre)clinical in vivo molecular imaging applications: adjusting the bullet to its target, Expert Opin. Biol. Ther. 13 (2013) 1149-1160. 
[183] M. D'Huyvetter, C. Xavier, V. Caveliers, et al., Radiolabeled nanobodies as theranostic tools in targeted radionuclide therapy of cancer, Expert Opin. Drug Deliv. (2014) 1-16.

[184] S. Oliveira, G.A. van Dongen, M. Stigter-van Walsum, et al., Rapid visualization of human tumor xenografts through optical imaging with a nearinfrared fluorescent anti-epidermal growth factor receptor nanobody, Mol. Imaging 11 (2011) 33-46.

[185] V. Cortez-Retamozo, M. Lauwereys, G. Hassanzadeh Gh, et al., Efficient tumor targeting by single-domain antibody fragments of camels, Int. J. Cancer 98 (2002) 456-462.
[186] R. Chakravarty, S. Goel, W. Cai, Nanobody: the "magic bullet" for molecular imaging? Theranostics 4 (2014) 386-398.

[187] I. Vaneycken, N. Devoogdt, N. Van Gassen, et al., Preclinical screening of antiHER2 nanobodies for molecular imaging of breast cancer, FASEB J. 25 (2011) 2433-2446.

[188] C. Vincke, R. Loris, D. Saerens, et al., General strategy to humanize a camelid single-domain antibody and identification of a universal humanized nanobody scaffold, J. Biol. Chem. 284 (2009) 3273-3284. 